\title{
SOLUCIÓN NUMÉRICA DE FLUIDOS VISCOSOS CON ELEMENTOS ISOPARAMÉTRICOS
}

TESIS QUE PRESENTA

\section{MARÍA LUISA SANDOVAL SOLÍS}

PARA LA OBTENCIÓN DEL GRADO DE MAESTRO EN MATEMÁTICAS

\begin{abstract}
ASESORES: DRA. PATRICIA SAAVEDRA BARRERA. DR. L. HÉCTOR JUÁREZ VALENCIA.
\end{abstract}

DICIEMBRE DE 1998

UNIVERSIDAD AUTÓNOMA METROPOLITANA-IZTAPALAPA DIVISIÓN DE CIENCIAS BÁSICAS E INGENIERÍA 
SOLUCIÓN NUMÉRICA DE FLUIDOS

VISCOSOS CON ELEMENTOS

ISOPARAMÉTRICOS

POR

MARÍA LUISA SANDOVAL SOLÍS

TESIS DE MAESTRÍA 
A la memoria

de mi padre

A mi madre y mis

hermanos por su

apoyo que para mi

es invaluable.

A mis amigos, en particular a Vicky. 


\section{Agradecimientos}

Agradezco de manera especial a la Dra. Patricia Saavedra y al Dr. L. Héctor Juárez por su apoyo y asesoramiento. En particular, al Dr. Juárez por su gran ayuda en momentos decisivos. Asimismo a la Dra. Rosa Ma. Velasco y al Dr. Francisco Javier Sánchez por las sugerencias, críticas y comentarios que le hicieron a este trabajo con el fin de mejorarlo.

También quiero agradecer a mis amigos, profesores y compañeros de trabajo su impulso, ayúda y compresión.

Finalmente, agradezco a CONACyT el apoyo económico que me brindó para realizar estos estudios. 


\section{Índice}

$\begin{array}{ll}\text { Introducción } & \mathbf{3}\end{array}$

1 Conceptos básicos y formulaciones débiles $\quad 8$

1.1 Espacios de funciones y normas. . . . . . . . . . . . . . . 9

1.2 Formulación débil del problema de Stokes. . . . . . . . . . . . . . . 11

1.3 Formulación débil del problema de Navier-Stokes. . . . . . . . . . . . . . . 15

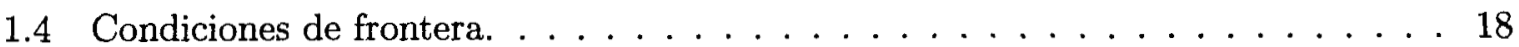

1.5 Métodos numéricos más comunes para resolver las ecuaciones de N-S para una formulación velocidad-presión. . . . . . . . . . . . . . . . . . . 19

1.5.1 Método integrado o mixto. . . . . . . . . . . . . . 20

1.5.2 Métodos de penalización. . . . . . . . . . . . . . . . 21

1.5.3 Método solenoidal o de divergencia libre. . . . . . . . . . . 23

2 Discretización de las ecuaciones de Navier-Stokes via el método del Elemento $\begin{array}{ll}\text { Finito } & 24\end{array}$

2.1 Discretización de un dominio poligonal. . . . . . . . . . . . . . 25

2.1 .1 Elemento de referencia y familia afín. . . . . . . . . . . . 27

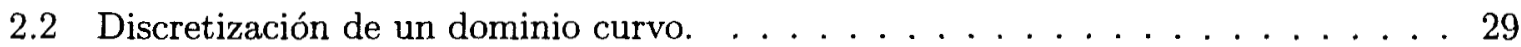

2.2 .1 Elementos y transformación isoparamétricos. . . . . . . . . . . . . . . 29

2.2 .2 Discretización de la velocidad y de la presión. . . . . . . . . . . . 33

2.3 Discretización de los problemas de Stokes y N-S . . . . . . . . . . . . . . 34

2.4 Definición de las matrices globales. . . . . . . . . . . . . . . . 37

2.5 Definición de las matrices locales. . . . . . . . . . . . . . . . . 40 
2.6 Definición de las funciones base locales y descripción de sus derivadas. . . . . . . 42

3 Métodos de solución y descripción del programa NAVSTOKE 46

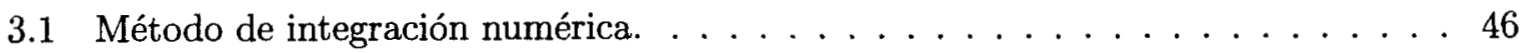

3.2 Método de almacenamiento de la matriz global A. . . . . . . . . . . 48

3.3 Métodos de resolución. . . . . . . . . . . . . . . . . . 49

3.3.1 Sistemas de ecuaciones lineales. . . . . . . . . . . . . . 50

3.3.2 Sistemas de ecuaciones no lineales. . . . . . . . . . . . . 50

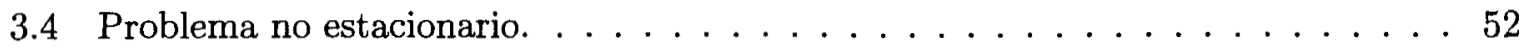

3.4.1 Esquema implícito de Euler hacia atrás. . . . . . . . . . . . 53

3.4 .2 Esquema de Punto Fijo. . . . . . . . . . . . . . . . . 53

3.5 Descripción del Programa NAVSTOKE . . . . . . . . . . . 54

3.5.1 Programas que discretizan el dominio $\bar{\Omega} \ldots \ldots \ldots \ldots$. . . . . . . . 54

3.5.2 Estructura del código NAVSTOKE. . . . . . . . . . . . 56

4 Ejemplos Numéricos $\quad 63$

4.1 Aplicación al flujo en una cavidad cuadrada. . . . . . . . . . . . . 63

4.1 .1 Problema de Stokes. . . . . . . . . . . . . . . . . . 64

4.1 .2 Problema de Navier-Stokes. . . . . . . . . . . . . . . 68

4.2 Aplicación al flujo alrededor de un cilindro cerca de una pared en movimiento. . 76

4.2 .1 Solución analítica. . . . . . . . . . . . . . . 77

4.2 .2 Solución numérica. . . . . . . . . . . . . . 78

4.3 Implantación. . . . . . . . . . . . . . . . 78

$\begin{array}{ll}\text { Conclusiones } & 81\end{array}$

$\begin{array}{ll}\text { Bibliografía } & 83\end{array}$ 


\section{Introducción}

El estudio del comportamiento de los flujos de fluidos viscosos es fundamental para el diseño y análisis de muchas clases de maquinaria y procesos de interés en la ingeniería. Por ejemplo, la lubricación es un proceso industrial muy importante, en donde la modelación matemática de los fluidos viscosos nos permiten analizar en forma rigurosa el comportamiento lubricante. Para este caso, presentamos su formulación matemática.

Supongamos que se va laminar un bloque de metal usando una plancha como guía de éste y un cilindro, veáse Figura 0-1. Si se quiere reducir la fricción que se produce al rodar el cilindro sobre el bloque grueso se necesita conservar un lubricante entre el cilindro y el metal, sobre todo en el precinto que es el punto en donde hay mayor presión, es decir, el lugar donde se reduce el grosor del bloque. Observemos que para lograr que exista este claro entre el cilindro y la guía del lubricante se requiere que el lubricante tenga cierta presión (grande) sobre el precinto.

Para obtener una primera aproximación del comportamiento del lubricante, se considera al lubricante como un fluido Newtoniano, con viscosidad constante y grande, e incompresible ( su densidad no varía respecto al tiempo y a la posición). Entonces su comportamiento se puede modelar mediante las siguientes ecuaciones adimensionadas:

Ecuaciones de Navier-Stokes

$$
\frac{\partial \mathbf{u}}{\partial t}+(\mathbf{u} \cdot \nabla) \mathbf{u}-\frac{1}{R e} \Delta \mathbf{u}+\nabla p=\mathbf{f} \quad \text { para } \quad \mathbf{x} \in \Omega, t>0
$$




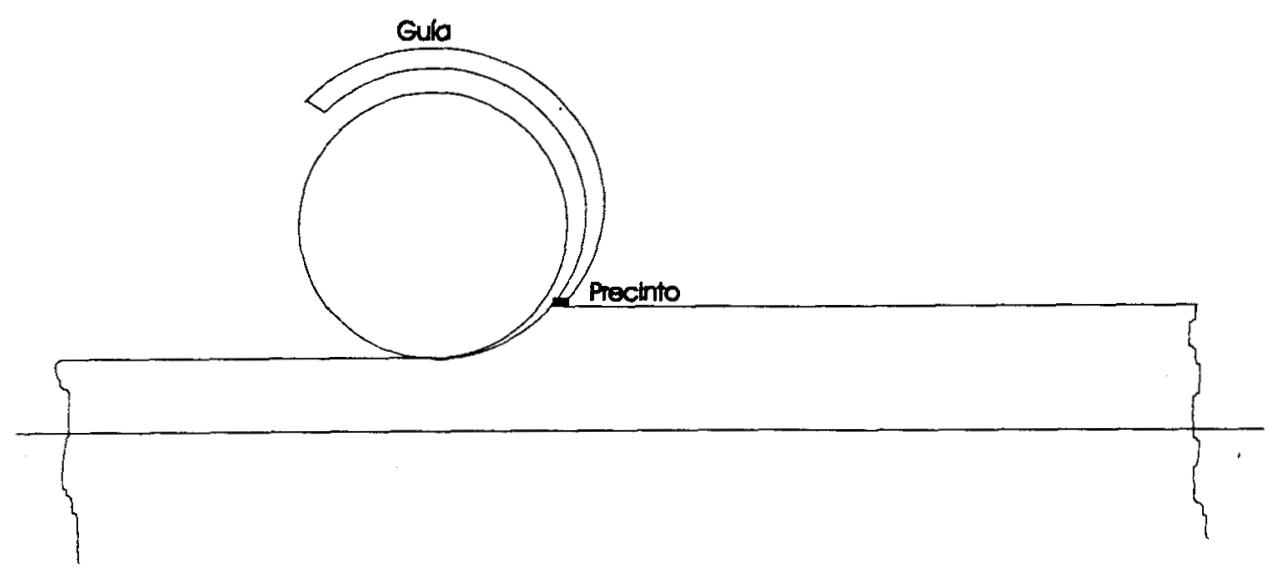

Figura 0-1: Laminación de un bloque de metal.

Ecuación de continuidad

$$
\boldsymbol{\nabla} \cdot \mathbf{u}=0 \text { para } \mathbf{x} \in \Omega, t>0
$$

+ condiciones de frontera

$$
\begin{aligned}
& \mathbf{u}(\mathbf{x}, t)=\mathbf{c}(\mathbf{x}, t) \quad \text { para } \quad \mathbf{x} \in \Gamma, t>0 \\
& \mathbf{u}(\mathbf{x}, 0)=\mathbf{u}_{0}(\mathbf{x}) \quad \text { para } \quad \mathbf{x} \in \Omega,
\end{aligned}
$$$$
+ \text { condiciones iniciales }
$$

en donde $\Omega \subset \mathbb{R}^{n}, n=2,3$ es la región donde fluye el fluido; $\Gamma$ es la frontera de $\Omega ; t \in[0, T]$ es el tiempo; $\mathbf{u}(\mathbf{x}, t)$ para $\mathbf{x} \in \Omega, t \geq 0$ es el campo de velocidades sobre $\Omega ; p(\mathbf{x}, t)$ es la presión; $\mathbf{f} \equiv \mathbf{f}(\mathbf{x}, t)$ es una fuerza volumétrica (como la fuerza de gravedad). Re es el número de Reynolds, definido por $R e=\frac{\rho L U}{\mu}$, donde $\rho$ es la densidad del fluido; $\mu$ es la viscosidad dinámica; $L$ es una longitud característica y $U$ es una velocidad característica.

Si el fluido es muy viscoso se observa que su movimiento es muy lento. Esto es debido a que las componentes de la inercia son muy pequeñas comparadas con las fuerzas viscosas. Por lo que, se puede despreciar el término convectivo de la ecuación (0.1), para obtener la igualdad

$$
\frac{\partial \mathbf{u}}{\partial t}-\frac{1}{R e} \Delta \mathbf{u}+\nabla p=\mathbf{f}
$$

Al sistema formado por las relaciones (0.4) y (0.2) se le conoce como las ecuaciones de Stokes y modelan el comportamiento de fluidos muy viscosos. 
Como el sistema no lineal (0.1) y el sistema lineal (0.4) involucran EDP (Ecuaciones Diferenciales Parciales) de segundo orden, se tienen que dar las condiciones iniciales y de frontera (0.3) para cerrar estos problemas. Cuando el problema es independiente del tiempo se dice que el movimiento del fluido está en estado estacionario y entonces el término $\frac{\partial \mathbf{u}}{\partial t}$ en las ecuaciones (0.1) y (0.4) es igual a cero, obteniendo así sistemas de ecuaciones de segundo orden elípticas, para las cuales sólo es necesario dar una condición de frontera en cada punto de $\Gamma$ para cada variable dependiente.

Por lo regular es muy complicado resolver este tipo de modelos en forma analítica (veánse Heywood [11], [12]), por ello se precisa hacerlo numéricamente. Observemos que si usamos el método de elemento finito para resolver numéricamente dicho problema u otros problemas sobre regiones con fronteras curvas, se pueden utilizar elementos con lados curvos en tal forma que se ajusten a la frontera de estudio. Por ello es muy importante que a un código de elemento finito que resuelva las ecuaciones (0.1), se le incorpore el uso de elementos isoparamétricos (de preferencia cuando el orden de los elementos sea bajo). La forma de hacer esto se explica en los apartados $(2.2 .1),(2.5)$ y (2.6) .

En la actualidad existen algunos paquetes comerciales, como FIDAP [8], que resuelven numéricamente las ecuaciones de Navier-Stokes. Con ellos se pueden resolver dichas ecuaciones usando los métodos numéricos más comunes. Sin embargo, funcionan como cajas negras, ya que uno selecciona un conjunto de subrutinas según el problema, se le proporcionan los datos y FIDAP obtiene los resultados, sin tener la posibilidad de cambiar, por ejemplo, el orden de los elementos finitos o el esquema numérico. $\mathrm{O}$ bien, cuando se trabajan las condiciones de frontera y las ecuaciones (0.1)-(0.2) en forma diferente a las usadas hasta ahora (veáse Saavedra [24]), se obtienen sistemas de ecuaciones que no están contemplados en estos paquetes. De ahí proviene la necesidad de contar con un código eficiente, para así conocer dónde y cómo hacer las posibles modificaciones.

Entre los métodos numéricos que hay para resolver las ecuaciones (0.1)-(0.2) se tienen los métodos que usan diferencias finitas como lo hacen Shih, Tan y Hwang [25] o los métodos que usan diferencias finitas para discretizar la variable temporal junto con la técnica de elemento finito para las variables espaciales. De estos últimos hay una gran variedad de opciones, entre ellas están las que trabajan con una formulación velocidad-presión, o con una formulación 
función corriente-vorticidad o simplemente con una formulación de corriente. A su vez, para cada una de estas formulaciones hay varios métodos numéricos que resuelven las ecuaciones de Navier-Stokes, en particular para la formulación velocidad-presión los más comunes son el método mixto o integrado, el método solenoidal o de divergencia libre y los métodos de penalización. En el apartado 1.5 se presenta un resumen de ellos incluyendo sus ventajas y desventajas.

En este trabajo se eligió el método mixto para resolver las ecuaciones de Navier-Stokes por ser el que aproxima simultáneamente a la velocidad y a la presión, por lo que, se utiliza un elemento mixto tipo Taylor-Hood. Además, se usa el método implícito de Euler hacia atrás para discretizar el término no estacionario, lo cual garantiza la estabilidad del esquema (veáse Temam [27]). Para resolver los problemas no lineales se escogió un método de punto fijo (veáse Juárez [16] para un análisis de convergencia de este método iterativo).

\section{Objetivos.}

1. El objetivo general de este trabajo es elaborar un programa (llamado NAVSTOKE) que resuelva las ecuaciones de Navier-Stokes en fluidos homogéneos, isotérmicos, incompresibles y viscosos usando la técnica de elemento finito cuando se tienen dominios en el plano con fronteras curvas y con condiciones de frontera tipo Dirichlet o Neumann.

2. Asimismo, se estudian las dificultades computacionales que pueden surgir al implantar el método mixto junto con el método implícito de Euler hacia atrás y el esquema de punto fijo.

3. Por otro lado, como resultado de 1. y 2. se obtiene un simulador numérico eficiente y preciso que permitirá posteriormente abordar otros problemas de mayor complejidad que involucren la resolución de las ecuaciones de Stokes o de Navier-Stokes como son los problemas de frontera libre acoplados con la ecuación de la energía.

Este trabajo se compone de 4 capítulos y conclusiones los cuales consisten en lo siguiente. En el capítulo 1 se presentan las formulaciones débiles tipo velocidad-presión de los problemas de Stokes y de Navier-Stokes, se define la terminología a utilizar y se discuten los resultados teóricos de existencia y unicidad. En el capítulo 2, se detalla la forma en que se discretizan las 
ecuaciones de Navier-Stokes vía el método de elemento finito. También, se definen los elementos isoparamétricos y cómo trabajan, ya que el código NAVSTOKE se concibió para regiones no estándar con fronteras curvas.

El capítulo 3 contiene una breve descripción del programa NAVSTOKE y los métodos de solución que se utilizan para resolver el problema no estacionario y el sistema de ecuaciones asociado.

Finalmente en el capítulo 4 se presentan dos problemas de prueba (benchmark) que se manejan en la literatura para validar el programa. Por último se exponen las conclusiones de este trabajo y la bibliografía utilizada. 


\section{Capítulo 1}

\section{Conceptos básicos y formulaciones}

\section{débiles}

Existen casos muy simples de problemas en los que se puede encontrar una solución analítica como son: el flujo de Couette, el flujo de Poiseuille, el flujo entre cilindros rotando, el flujo de canales convergentes y divergentes, el flujo sobre paredes porosas, veáse [6] para una descripción de cada uno de estos problemas. Para problemas más complejos no es posible encontrar soluciones analíticas, por lo cual se recurre a los métodos numéricos. Como en este trabajo se utilizará el método de Elemento Finito, se debe reescribir el problema (0.1)-(0.2) a través de una formulación débil, la cual debilita las condiciones de regularidad de las variables que intervienen en dichos modelos, para así tener una nueva formulación del problema en espacios más generales. Los espacios de funciones más comunes utilizados en este tipo de formulaciones son los espacios de Sobolev (veánse [9] y [21]). Hay varias clases de formulaciones para las ecuaciones dinámicas de los fluidos, entre ellas están: la formulación velocidad-presión, la formulación función corriente-vorticidad y la formulación de corriente.

En este capítulo se presentan las formulaciones débiles de los problemas (0.1)-(0.2) y (0.4)(0.2) del tipo velocidad-presión, así como las condiciones necesarias para la existencia y unicidad de sus soluciones generalizadas (débiles). Además, se presentan las distintas clases de condi- 
ciones de frontera consideradas en esta tesis y cuales son los métodos numéricos más comunes para resolver dichos problemas.

\subsection{Espacios de funciones y normas.}

Para dar las formulaciones débiles más comunes de los problemas (0.1)-(0.2) y (0.4)-(0.2) es necesario definir los siguientes espacios de funciones, así como sus normas. Para ello se considera que el dominio $\Omega$ es un subconjunto abierto de $\mathbb{R}^{n}, n=2$ o 3 , conexo y acotado, cuya frontera $\Gamma$ es Lipschitz-continua, es decir que admite un vector tangente en cada punto de $\Omega$.

$\mathrm{Al}$ espacio lineal que consiste de todas las funciones $v: \mathbb{R}^{n} \rightarrow \mathbb{R}$ cuyas derivadas parciales $\partial^{\alpha} v$ de ordenes $0 \leq \alpha \leq m$ sean continuas sobre $\Omega$ se le denota por $C^{m}(\Omega)$. Por $C_{c}^{m}(\Omega)$ se conoce al subespacio lineal de $C^{m}(\Omega)$ que está formado por las funciones escalares $v \in C^{m}(\Omega)$ y que tienen soporte compacto sobre $\Omega$. El soporte de $v$ se define como

$$
\text { soporte de } v \equiv \overline{\{\mathrm{x} \in \Omega \mid v(\mathbf{x}) \neq 0\}} \text {. }
$$

Para espacios de funciones vectoriales se usará la notación en negrita. Por ejemplo por $\mathbf{C}_{c}^{m}(\Omega)$ se entenderá $n$ copias de $C_{c}^{m}(\Omega)$, es decir $\mathbf{C}_{c}^{m}(\Omega)=\left[C_{\mathrm{c}}^{m}(\Omega)\right]^{n}$.

$\mathrm{El}$ espacio constituido por funciones medibles que son cuadrado integrables sobre $\Omega$ es denotado por $L^{2}(\Omega)$ y tiene asociada la norma

$$
\|q\|_{0}=(q, q)^{1 / 2}, \quad \text { en donde } \quad(p, q)=\int_{\Omega} p q d \Omega
$$

A partir de este espacio se define

$$
L_{0}^{2}(\Omega)=\left\{q \in L^{2}(\Omega) \mid \int_{\Omega} q d \Omega=0\right\}
$$

El espacio de Sobolev $H^{1}(\Omega)$ está formado por todas las funciones que pertenecen a $L^{2}(\Omega)$ y que su primera derivada en el sentido de distribuciones pertenece también a $L^{2}(\Omega)$. La norma 
asociada a dicho espacio es

$$
\|v\|_{1}=\left(\|v\|_{0}^{2}+\sum_{i=1}^{n}\left\|\frac{\partial v}{\partial x_{i}}\right\|_{0}^{2}\right)^{1 / 2}
$$

recordando que $\Omega \subset \mathbb{R}^{n}$, con $n=2$ o 3 . Un subespacio de $H^{1}(\Omega)$ es

$$
H_{0}^{1}(\Omega)=\left\{v \in H^{1}(\Omega) \mid v=0 \text { sobre } \Gamma\right\}
$$

para el cual una norma es

$$
|v|_{1}=\left(\sum_{i=1}^{n}\left\|\frac{\partial v}{\partial x_{i}}\right\|_{0}^{2}\right)^{1 / 2}
$$

que es equivalente a la norma $\|\cdot\|_{1}$ descrita en (1.3) por la desigualdad de Poincaré-Friedrichs (veáse Ciarlet [5]).

Denotaremos por $H^{-1}(\Omega)$ al espacio dual de $H_{0}^{1}(\Omega)$, es decir, consiste de todas las funcionales lineales sobre $H_{0}^{1}(\Omega)$ que están acotadas. Una norma para $H^{-1}(\Omega)$ está dada por

$$
\|f\|_{-1}=\sup _{0 \neq v \in H_{0}^{1}(\Omega)} \frac{(f, v)}{|v|_{1}}
$$

En este trabajo se estudiará el campo de velocidades en dos dimensiones, por lo que se define el siguiente subespacio

$$
\mathbf{H} d i v=\left\{\mathbf{v} \in \mathbf{H}_{0}^{1}(\Omega) \mid \boldsymbol{\nabla} \cdot \mathbf{v}=0 \text { en } \Omega\right\}
$$

en donde $\mathbf{H}_{0}^{1}(\Omega) \equiv\left[H_{0}^{1}(\Omega)\right]^{n}$. Es fácil demostrar que $\mathbf{H}$ div es un espacio de Hilbert para la norma de $\mathbf{H}_{0}^{1}(\Omega)$, o sea,

$$
(\mathbf{u}, \mathbf{v})_{\mathbf{H} d i v}=\sum_{i, j=1}^{n}\left(\frac{\partial u_{i}}{\partial x_{j}}, \frac{\partial v_{i}}{\partial x_{j}}\right), \quad\|\mathbf{v}\|_{\mathbf{H} d i v}=(\mathbf{v}, \mathbf{v})_{\mathbf{H} d i v}^{\frac{1}{2}}
$$

Sea $X$ un espacio de Banach de funciones definidas de $\mathbb{R}^{n}$ a $\mathbb{R}$. El espacio de Banach $L^{2}(0, T ; X)$ está formado por el conjunto de funciones $v=v(\mathbf{x}, t)$ tales que $v(\mathbf{x}, t) \in X$ para 
cada $t \in[0, T]$, y $\int_{0}^{T}\|v(\cdot, t)\|_{X}^{2} d t<\infty$. Este espacio tiene asociada la norma

$$
\|v\|_{L^{2}(0, T ; X)}=\left\{\int_{0}^{T}\|v(\cdot, t)\|_{X}^{2} d t\right\}^{\frac{1}{2}}
$$

Análogamente, se define el espacio $L^{\infty}(0, T ; X)$ formado por las funciones medibles y acotadas en $[0, T]$ excepto por un conjunto de medida cero (funciones acotadas esencialmente "ess") con norma

$$
\|v\|_{L^{\infty}(0, T ; X)}=e s s \sup _{t \in[0, T]}\|v(\cdot, t)\|_{X}
$$

El espacio $C([0, T] ; X)$ está formado por funciones continuas que van de $[0, T]$ a $X$. Si $T<\infty$ su norma es

$$
\sup _{t \in[0, T]}\|f(t)\|_{X}
$$

en donde $X$ es un espacio de Banach.

Por último, denotaremos por $\mathfrak{L}(\mathbf{A}, \mathbf{B})$ al conjunto de todos los operadores lineales continuos del espacio vectorial $\mathbf{A}$ en el espacio vectorial $\mathbf{B}$.

\subsection{Formulación débil del problema de Stokes.}

El problema de Stokes con condiciones de Dirichlet homogéneas y condiciones iniciales está formado por las ecuaciones $(0.4),(0.2)$ junto con

$$
\begin{array}{rlr}
\mathbf{u} & =\mathbf{0} & \text { sobre } \Gamma, \\
\mathbf{u}(\mathbf{x}, 0) & =\mathbf{u}_{0}(\mathbf{x}) . &
\end{array}
$$

Para hallar una formulación débil de este problema, multiplicamos $(0.4)$ por $\mathbf{v} \in \mathrm{C}_{c}^{1}(\Omega)$ e integramos sobre $\Omega$, para obtener

$$
\int_{\Omega}\left(\frac{\partial \mathbf{u}}{\partial t}-\frac{1}{R e} \Delta \mathbf{u}+\nabla p\right) \cdot \mathbf{v} d \Omega=\int_{\Omega} \mathbf{f} \cdot \mathbf{v} d \Omega \quad \forall \mathbf{v} \in \mathbf{C}_{c}^{1}(\Omega)
$$

Al aplicar las fórmulas de Green $\left(\int_{\Omega} \nabla \mathbf{u} \cdot \nabla \mathbf{v} d \Omega=-\int_{\Omega} \Delta \mathbf{u} \cdot \mathbf{v} d \Omega+\int_{\Gamma} \mathbf{v} \cdot \frac{\partial \mathbf{u}}{\partial \mathbf{n}} d \Gamma\right)$ a los 
términos del lado izquierdo de la ecuación anterior, tomando en cuenta que $\mathbf{v}=\mathbf{0}$ sobre $\Gamma$ y que $\mathrm{C}_{c}^{1}(\Omega)$ es denso sobre $\mathbf{H}_{0}^{1}(\Omega)$ se tiene que

$$
\frac{d}{d t} \int_{\Omega} \mathbf{u} \cdot \mathbf{v} d \Omega+\int_{\Omega}\left(\frac{1}{R \boldsymbol{e}} \nabla \mathbf{u} \cdot \nabla \mathbf{v}\right) d \Omega-\int_{\Omega}(p \boldsymbol{\nabla} \cdot \mathbf{v}) d \Omega=\int_{\Omega} \mathbf{f} \cdot \mathbf{v} d \Omega \quad \forall \mathbf{v} \in \mathbf{H}_{0}^{1}(\Omega),
$$

en donde $\int_{\Omega} \frac{\partial \mathbf{u}}{\partial t} \cdot \mathbf{v} d \Omega=\frac{d}{d t} \int_{\Omega} \mathbf{u} \cdot \mathbf{v} d \Omega$

Para la ecuación de continuidad (0.2), multiplicamos ésta por $q \in L_{0}^{2}(\Omega)$ e integramos sobre $\Omega$, así

$$
\int_{\Omega}(q \nabla \cdot \mathbf{u}) d \Omega=0 \quad \forall q \in L_{0}^{2}(\Omega)
$$

Por lo tanto, el problema definido en $(0.4),(0.2)$ y (1.8) tiene la siguiente formulación variacional:

$$
\begin{gathered}
\text { Encontrar }(\mathbf{u}, p) \in \mathbf{L}^{2}\left(0, T ; \mathbf{H}_{0}^{1}(\Omega)\right) \times L^{2}\left(0, T ; L_{0}^{2}(\Omega)\right) \quad \text { tal que } \\
\begin{array}{c}
\frac{d}{d t}(\mathbf{u}, \mathbf{v})+a(\mathbf{u}, \mathbf{v})+b(p, \mathbf{v})=L_{f}(\mathbf{v}) \quad \forall \mathbf{v} \in \mathbf{H}_{0}^{1}(\Omega), \\
b(q, \mathbf{u})=0 \quad \forall q \in L_{0}^{2}(\Omega), \\
\mathbf{u}(\mathbf{x}, 0)=\mathbf{u}_{0}(\mathbf{x})
\end{array}
\end{gathered}
$$

en donde

$$
\begin{gathered}
(\mathbf{u}, \mathbf{v})=\sum_{i=1}^{n} \int_{\Omega} u_{i} v_{i} d \Omega \\
a(\mathbf{u}, \mathbf{v})=\frac{1}{R e} \sum_{i, j=1}^{n} \int_{\Omega} \frac{\partial u_{i}}{\partial x_{j}} \frac{\partial v_{i}}{\partial x_{j}} d \Omega \\
b(p, \mathbf{v})=-\sum_{i=1}^{n} \int_{\Omega} p \frac{\partial v_{i}}{\partial x_{i}} d \Omega \\
L_{f}(\mathbf{v})=\sum_{i=1}^{n} \int_{\Omega} f_{i} v_{i} d \Omega .
\end{gathered}
$$

Observemos que en la formulación (1.11) las variables $\mathbf{u}$ y $p$ están acopladas y que se encuentran en espacios distintos, por lo cual se requiere de un método mixto para resolverla numéricamente (veáse apartado 1.5.1). Por otro lado, si se quiere resolver el problema de Stokes con un método solenoidal o de divergencia libre (veáse apartado 1.5.3) se necesita una formulación distinta a la dada en (1.11). Para encontrar dicha formulación se multiplica la igualdad (0.4) por una función prueba $\mathbf{v} \in \mathbf{H} d i v$, entonces la ecuación (0.4) tiene la siguiente forma

$$
\frac{d}{d t} \int_{\Omega} \mathbf{u} \cdot \mathbf{v} d \Omega+\int_{\Omega} \frac{1}{R e} \nabla \mathbf{u} \cdot \nabla \mathbf{v} d \Omega=\int_{\Omega} \mathbf{f} \cdot \mathbf{v} d \Omega \quad \forall \mathbf{v} \in \mathbf{H} d i v
$$


Luego, una nueva formulación débil para el problema $(0.4),(0.2)$ y (1.8) se enuncia de la siguiente manera:

$$
\begin{gathered}
\text { Encontrar } \mathbf{u} \in \mathbf{L}^{2}(0, T ; \mathbf{H} d i v) \quad \text { tal que } \\
\begin{aligned}
\frac{d}{d t}(\mathbf{u}, \mathbf{v})+a(\mathbf{u}, \mathbf{v}) & =L_{f}(\mathbf{v}) \quad \forall \mathbf{v} \in \mathbf{H} d i v \\
\mathbf{u}(\mathbf{x}, 0) & =\mathbf{u}_{0}(\mathbf{x})
\end{aligned}
\end{gathered}
$$

Con respecto a la unicidad y existencia de la solución de las formulaciones (1.11) y (1.14) sin el término $\frac{d}{d t}(\mathbf{u}, \mathbf{v})$, es decir, para el problema estacionario se tiene el siguiente teorema:

Teorema 1.1. Sea $\Omega$ un subconjunto abierto de $\mathbb{R}^{n}$, conexo y acotado con frontera $\Gamma$ Lipschitz-continua. Dado Re $>0$ y $\mathbf{f} \in \mathbf{H}^{-1}(\Omega)$ existe un único par de funciones $(\mathbf{u}, p) \in$ $\mathbf{H}_{0}^{1}(\Omega) \times L_{0}^{2}(\Omega)$ tal que

$$
\begin{gathered}
a(\mathbf{u}, \mathbf{v})+b(p, \mathbf{v})=L_{f}(\mathbf{v}) \quad \forall \mathbf{v} \in \mathbf{H}_{0}^{1}(\Omega), \\
b(q, \mathbf{u})=0 \quad \forall q \in L_{0}^{2}(\Omega) .
\end{gathered}
$$

Demostración. Veáse Girault y Raviart [9] para los detalles de la demostración.

A fin de dar un resumen de la demostración del teorema es necesario definir los siguientes conceptos:

La propiedad de la forma bilineal $a(\cdot, \cdot)$ de ser $\mathbf{H}$ div-elíptica significa que existe una constante $\alpha>0$ tal que

$$
a(\mathbf{v}, \mathbf{v}) \geq \alpha\|\mathbf{v}\|_{\mathbf{H} d i v}^{2} \quad \forall \mathbf{v} \in \mathbf{H} d i v
$$

Si existe una constante $\beta>0$ tal que se cumpla la siguiente desigualdad

$$
\inf _{q \in L_{0}^{2}(\Omega)} \sup _{\mathbf{v} \in \mathbf{H}_{0}^{1}(\Omega)} \frac{(q, \operatorname{div} \mathbf{v})}{|\mathbf{v}|_{1}\|q\|_{0}} \geq \beta \quad \forall q \in L_{0}^{2}(\Omega)
$$

entonces decimos que se satisface la condición inf-sup.

Observemos que a las formas bilineales $a(\cdot, \cdot)$ y $b(\cdot, \cdot)$ se les puede asociar dos operadores lineales $\mathbf{A} \in \mathfrak{L}\left(\mathbf{H}_{0}^{1}(\Omega), \mathbf{H}^{-1}(\Omega)\right)$ y $\mathbf{B} \in \mathfrak{L}\left(L_{0}^{2}(\Omega), L_{0}^{2}(\Omega)\right)$, respectivamente, tal que:

$$
\begin{aligned}
\langle\mathbf{A u}, \mathbf{v}\rangle & =a(\mathbf{u}, \mathbf{v}) \quad \forall \mathbf{u}, \mathbf{v} \in \mathbf{H}_{0}^{1}(\Omega), \\
\langle\mathbf{B v}, p\rangle & =b(p, \mathbf{v}) \quad \forall \mathbf{v} \in \mathbf{H}_{0}^{1}(\Omega), \quad \forall q \in L_{0}^{2}(\Omega) .
\end{aligned}
$$


Además definiremos a $\mathbf{B}^{\prime} \in \mathfrak{L}\left(L_{0}^{2}(\Omega), L_{0}^{2}(\Omega)\right)$ como el operador dual de $\mathbf{B}$, tal que

$$
\left\langle\mathbf{B}^{\prime} p, \mathbf{v}\right\rangle=\langle p, \mathbf{B v}\rangle=b(p, \mathbf{v}) \quad \forall \mathbf{v} \in \mathbf{H}_{0}^{1}(\Omega), \quad \forall q \in L_{0}^{2}(\Omega)
$$

Al usar estos operadores, el problema (1.15) se puede hacer equivalente al siguiente problema:

$$
\begin{gathered}
\text { Encontrar }(\mathbf{u}, p) \in \mathbf{H}_{0}^{1}(\Omega) \times L_{0}^{2}(\Omega) \text { tal que } \\
\begin{aligned}
\mathbf{A} \mathbf{u}+\mathbf{B}^{\prime} p=\mathbf{f} \quad \text { en } \quad \mathbf{H}^{-1}(\Omega), \\
\mathbf{B u}=0 \quad \text { en } \quad L_{0}^{2}(\Omega) .
\end{aligned}
\end{gathered}
$$

La idea de la demostración es probar que el problema (1.15) está bien planteado, es decir, se debe mostrar que el operador lineal $\Phi: \mathbf{H}_{0}^{1}(\Omega) \times L_{0}^{2}(\Omega) \rightarrow \mathbf{H}^{-1}(\Omega) \times L_{0}^{2}(\Omega)$ definido por

$$
\Phi(\mathbf{v}, q)=\left(\mathbf{A v}+\mathbf{B}^{\prime} q, \mathbf{B} \mathbf{v}\right)
$$

es un isomorfismo entre $\mathbf{H}_{0}^{1}(\Omega) \times L_{0}^{2}(\Omega)$ y $\mathbf{H}^{-1}(\Omega) \times L_{0}^{2}(\Omega)$. Para ello, se verifica primero que si la pareja $(\mathbf{u}, p)$ es solución del problema (1.15) entonces $\mathbf{u}$ es solución del problema (1.14) sin el término temporal, y para probar el recíproco de la proposición se sigue este razonamiento: si u es solución del problema estacionario correspondiente a (1.14) y si además la forma bilineal $a(\cdot, \cdot)$ es $\mathbf{H}$ div-elíptica y $b(\cdot, \cdot)$ satisface la condición inf-sup, se muestra que para cada $\mathbf{f} \in \mathbf{H}^{-1}(\Omega)$ existe un único $p$ tal que el par de funciones $(\mathbf{u}, p)$ es la única solución del problema (1.18) y por lo tanto $\Phi$ es biyectiva, o sea es un isomorfismo entre $\mathbf{H}_{0}^{1}(\Omega) \times L_{0}^{2}(\Omega)$ y $\mathbf{H}^{-1}(\Omega) \times L_{0}^{2}(\Omega)$.

Ahora se tiene el siguiente resultado que muestra la existencia y unicidad de la solución del problema no estacionario dado en (1.14):

Teorema 1.2. Sea $\Omega$ un subconjunto abierto de $\mathbb{R}^{n}$, conexo y acotado con frontera $\Gamma$ Lipschitz-continua $y$ sea $\mathbf{W}=\left\{\mathbf{v} \in \mathbf{L}^{2}(\Omega) \mid \boldsymbol{\nabla} \cdot \mathbf{v}=0\right.$ en $\left.\Omega\right\}$. Dado Re $>0, \mathbf{u}_{0} \in \mathbf{W}$ y $\mathbf{f} \in \mathbf{L}^{2}\left(0, T ;(\mathbf{H} d i v)^{\prime}\right)$, entonces existe una única función $\mathbf{u} \in \mathbf{L}^{2}(0, T ; \mathbf{H}$ div $)$, la cual satisface (1.14) y $\mathbf{u}(\mathbf{x}, 0)=\mathbf{u}_{0}(\mathbf{x})$. Más aún $\mathbf{u} \in \mathbf{C}([0, T] ; \mathbf{W})$.

Demostración. La prueba de este teorema se puede verificar en Temam [27]. 


\subsection{Formulación débil del problema de Navier-Stokes.}

A continuación se darán las formulaciones débiles más comunes de las ecuaciones de NavierStokes. Para ello se consideran condiciones de frontera de tipo Dirichlet homogéneas, es decir, el problema está formado por las ecuaciones $(0.1),(0.2)$ y (1.8).

Estas formulaciones débiles se encuentran en forma análoga al caso de las ecuaciones de Stokes, es decir, se obtiene el producto escalar de $(0.1)$ con $\mathbf{v} \in \mathbf{H}_{0}^{1}(\Omega)$, se aplica la fórmula de Green y se utiliza que $\mathbf{v}=\mathbf{0}$ sobre $\Gamma$. Así, se obtiene la siguiente formulación débil de $(0.1),(0.2)$ y $(1.8)$ :

$$
\begin{gathered}
\text { Encontrar }(\mathbf{u}, p) \in \mathbf{L}^{2}\left(0, T ; \mathbf{H}_{0}^{1}(\Omega)\right) \times L^{2}\left(0, T ; L_{0}^{2}(\Omega)\right) \text { tal que } \\
\begin{array}{c}
\frac{d}{d t}(\mathbf{u}, \mathbf{v})+a_{1}(\mathbf{u} ; \mathbf{u}, \mathbf{v})+a(\mathbf{u}, \mathbf{v})+b(p, \mathbf{v})=L_{f}(\mathbf{v}) \quad \forall \mathbf{v} \in \mathbf{H}_{0}^{1}(\Omega) \\
b(q, \mathbf{u})=0 \quad \forall q \in L_{0}^{2}(\Omega) \\
\mathbf{u}(\mathbf{x}, 0)=\mathbf{u}_{0}(\mathbf{x})
\end{array}
\end{gathered}
$$

en donde

$$
\begin{gathered}
a_{1}(\mathbf{u} ; \mathbf{w}, \mathbf{v})=\sum_{i, j=1}^{n} \int_{\Omega} u_{j}\left(\frac{\partial w_{i}}{\partial x_{j}}\right) v_{i} d \Omega \\
a(\mathbf{u}, \mathbf{v})=\frac{1}{R e} \sum_{i, j=1}^{n} \int_{\Omega} \frac{\partial u_{i}}{\partial x_{j}} \frac{\partial v_{i}}{\partial x_{j}} d \Omega \\
b(p, \mathbf{v})=-\sum_{i=1}^{n} \int_{\Omega} p \frac{\partial v_{i}}{\partial x_{i}} d \Omega \\
L_{f}(\mathbf{v})=\sum_{i=1}^{n} \int_{\Omega} f_{i} v_{i} d \Omega
\end{gathered}
$$

Nuevamente, si se quieren desacoplar las variables $\mathbf{u}$ y $p$ se utilizan funciones de prueba $\mathbf{v}$ en $\mathbf{H} d i v$, entonces el problema (1.19) se transforma en lo siguiente:

$$
\begin{aligned}
& \text { Encontrar } \mathbf{u} \in \mathbf{L}^{2}(0, T ; \mathbf{H} d i v) \quad \text { tal que } \\
& \begin{aligned}
\frac{d}{d t}(\mathbf{u}, \mathbf{v})+a(\mathbf{u} ; \mathbf{u}, \mathbf{v}) & =L_{f}(\mathbf{v}) \quad \forall \mathbf{v} \in \mathbf{H} d i v \\
\mathbf{u}(\mathbf{x}, 0) & =\mathbf{u}_{0}(\mathbf{x})
\end{aligned}
\end{aligned}
$$

en donde

$$
\mathrm{a}(\mathbf{u} ; \mathbf{u}, \mathbf{v})=a_{1}(\mathbf{u} ; \mathbf{u}, \mathbf{v})+a(\mathbf{u}, \mathbf{v})
$$

La formulación variacional (1.21) del problema de Navier-Stokes se utiliza cuando se encuentra primero la velocidad ( o si solamente se quiere encontrar la velocidad) y después la presión, usando el método de divergencia libre (veáse el apartado 1.5.3). 
Respecto a la existencia y unicidad de la solución para la formulación débil del problema estacionario (1.19) sin el término temporal, se tiene el teorema siguiente:

Teorema 1.3. Sea $n \leq 4$ y $\Omega$ un dominio (subconjunto abierto y conexo) y acotado en $\mathbb{R}^{n}$ con frontera $\Gamma$ Lipschitz-continua. Dada $\mathbf{f} \in \mathbf{H}^{-1}(\Omega)$, existe al menos una pareja $(\mathbf{u}, p) \in \mathbf{H}_{0}^{1}(\Omega) \times \mathbf{L}_{0}^{2}(\Omega)$ la cual satisface

$$
\begin{gathered}
a_{1}(\mathbf{u} ; \mathbf{u}, \mathbf{v})+a(\mathbf{u}, \mathbf{v})+b(p, \mathbf{v})=L_{f}(\mathbf{v}) \quad \forall \mathbf{v} \in \mathbf{H}_{0}^{1}(\Omega) \\
b(q, \mathbf{u})=0 \quad \forall q \in L_{0}^{2}(\Omega)
\end{gathered}
$$

o equivalentemente las ecuaciones $(0.1),(0.2)$ y (1.8). Además si se pide que

$$
\begin{gathered}
\left(\operatorname{Re}^{2} \mathcal{N}\right)\|\mathbf{f}\|_{\mathbf{H} d i v^{\prime}}<1, \\
\operatorname{con} \quad \mathcal{N}=\sup _{\mathbf{u}, \mathbf{v} \in \mathbf{H} d i v} \frac{a_{1}(\mathbf{u} ; \mathbf{u}, \mathbf{v})}{|\mathbf{u}|_{1, \Omega}|\mathbf{v}|_{1, \Omega}|\mathbf{u}|_{1, \Omega}} \quad y \quad\|\mathbf{f}\|_{\mathbf{H} d i v^{\prime}}=\sup _{\mathbf{v} \in \mathbf{H} d i v} \frac{(\mathbf{f}, \mathbf{v})}{|\mathbf{v}|_{1, \Omega}}
\end{gathered}
$$

entonces el problema (1.22) tiene una única solución $(\mathbf{u}, p)$ en $\mathbf{H}_{0}^{1}(\Omega) \times \mathbf{L}_{0}^{2}(\Omega)$.

Demostración. Los detalles de la demostración están en Girault y Raviart [9].

La idea de la demostración es análoga a la del teorema 1.1, o sea, se muestra que si $(\mathbf{u}, p)$ es solución del problema estacionario (1.22) entonces $\mathbf{u}$ también es solución del problema estacionario correspondiente a (1.21), y la propiedad inversa se verifica a partir de que la forma bilineal $b(\because)$ satisface la condición inf-sup (veáse relación (1.17)). Lo único que faltaría comprobar es que efectivamente el problema no lineal (1.21) tiene una solución única, está parte es la más importante de la demostración.

Para verificar que el problema (1.21) tiene al menos una solución se construye una sucesión de soluciones aproximadas $\left\{\mathbf{u}_{m}\right\}$ usando el método de Galerkin, es decir, para cada $m \geq 1$ existe un subespacio $\mathbf{V}_{m}$ de $\mathbf{H} d i v$ tal que la $\mathbf{u}_{m}$ es solución de un problema aproximado al problema estacionario correspondiente a $(1.21)$, llamado problema $\left(\mathrm{P}_{m}\right)$ :

$$
\begin{array}{cl}
\text { Encontrar } \mathbf{u}_{m} \in \mathbf{V}_{m} & \text { tal que } \\
\mathrm{a}\left(\mathbf{u}_{m} ; \mathbf{u}_{m}, \mathbf{v}\right)=L_{f}(\mathbf{v}) \quad \forall \mathbf{v} \in \mathbf{V}_{m}
\end{array}
$$

Ahora, para probar que el problema $\left(\mathrm{P}_{m}\right)$ tiene una solución $\mathbf{u}_{m}$, se utiliza una consecuencia 
del teorema del punto fijo de Brouwer (veáse [9]) y el hecho de que la forma bilineal a (w,,$\cdot \cdot)$ es uniformemente $\mathbf{H} d i v$-elíptica, o sea que exite $\alpha>0$ tal que

$$
\mathrm{a}(\mathbf{w}, \mathbf{v}, \mathbf{v}) \geq \alpha\|\mathbf{v}\|_{\mathbf{H} d i v} \quad \forall \mathbf{v}, \mathbf{w} \in \mathbf{H} d i v .
$$

Finalmente para probar que la sucesión $\left\{\mathbf{u}_{m}\right\}$ converge fuertemente a una solución $\mathbf{u}$ del problema (1.21) se ocupa la compacidad del encajamiento (embedding) de $\mathbf{H}_{0}^{1}$ en $\mathbf{L}_{0}^{2}(\Omega)$.

Por otro lado, para demostrar la unicidad de la solución del problema estacionario (1.21), observemos que

$$
\begin{aligned}
\mathbf{a}(\mathbf{u} ; \mathbf{u}, \mathbf{v}) & =\langle\mathbf{A}(\mathbf{u}) \mathbf{u}, \mathbf{v}\rangle=\langle\mathbf{f}, \mathbf{v}\rangle \\
& =\langle\pi \mathbf{A}(\mathbf{u}) \mathbf{u}, \mathbf{v}\rangle=\langle\pi \mathbf{f}, \mathbf{v}\rangle
\end{aligned}
$$

en donde $\pi: \mathbf{H}^{-1} \rightarrow \mathbf{H} d i v^{\prime}$ y $\mathbf{A}(\mathbf{u}): \mathbf{H}_{0}^{1} \rightarrow \mathbf{H}^{-1}, \mathbf{A}(\mathbf{u}) \mathbf{u}$ en $\mathbf{H}^{-1}$ y $\mathbf{A}$ se definió en (1.17') Así el problema (1.21) se puede reescribir como:

$$
\begin{gathered}
\text { Encontrar } \mathbf{u} \in \mathbf{H} \text { div tal que } \\
\pi \mathbf{A}(\mathbf{u}) \mathbf{u}=\pi \mathbf{f} \quad \text { en } \mathbf{H} \text { div }^{\prime} \quad \text { ó bien } \quad \mathbf{u}=\mathbf{T}(\mathbf{u}) \pi \mathbf{f} \text { en } \mathbf{H} \text { div }
\end{gathered}
$$

en donde $\mathbf{T}(\mathbf{u})$ es el mapeo inverso de $\pi \mathbf{A}(\mathbf{u})$. La idea se basa en mostrar que la transformación $\mathbf{v} \rightarrow \mathbf{T}(\mathbf{v}) \pi \mathbf{f}$ es una contracción, es decir, que la transformación tiene un único punto fijo si se cumple la desigualdad (1.23) y la relación siguiente

$$
\|(\mathbf{T}(\mathbf{u})-\mathbf{T}(\mathbf{v})) \pi \mathbf{f}\|_{\mathbf{H}_{0}^{1}} \leq\left(R e^{2} \mathcal{N}\right)\|\mathbf{f}\|_{\mathbf{H} d i v^{\prime}}\|\mathbf{u}-\mathbf{v}\|_{\mathbf{H}_{0}^{1}}
$$

Para ello se necesita que la forma bilineal $a(\mathbf{w}, \cdot, \cdot)$ sea uniformemente $\mathbf{H}$ div-elíptica (1.16) y que el mapeo $\mathbf{w} \rightarrow \pi \mathbf{A}(\mathbf{w})$ sea localmente Lipschitz-continuo en $\mathbf{H} d i v$.

Enseguida, el teorema 1.4 enuncia las condiciones para que exista la solución del problema no estacionario (1.21) y el teorema 1.5 da la condición para que sea única.

Teorema 1.4. Sea $n \leq 4$ y $\Omega$ un dominio acotado en $\mathbb{R}^{n}$ con frontera $\Gamma$ Lipschitzcontinua y sea $\mathbf{W}=\left\{\mathbf{v} \in \mathbf{L}^{2}(\Omega) \mid \nabla \cdot \mathbf{v}=0\right.$ en $\left.\Omega\right\}$. Dado Re $>0, \mathbf{u}_{0} \in \mathbf{W}$ y $\mathbf{f} \in \mathbf{L}^{2}\left(0, T ; \mathbf{H} d i v^{\prime}\right)$, 
entonces existe al menos una función $\mathbf{u} \in \mathbf{L}^{2}(0, T ; \mathbf{H}$ div $)$ que satisface $(1.21)$ y $\mathbf{u}(\mathbf{x}, 0)=$ $\mathbf{u}_{0}(\mathbf{x})$. Más aún $\mathbf{u} \in \mathbf{L}^{\infty}(0, T ; \mathbf{W})$ y u es débilmente continua de $[0, T]$ a $\mathbf{W}$, es decir, $\forall \mathbf{v} \in \mathbf{W}, t \rightarrow(\mathbf{u}(t), \mathbf{v})$ es una función escalar continua.

Demostración. En Temam [27] se puede ver la demostración.

Teorema 1.5. En el caso de dos dimensiones la solución dada en el teorema 1.4 es única. Además u es una función continua casi donde sea $y \mathbf{u}(t) \rightarrow \mathbf{u}_{0}$, en $\mathbf{W}$, cuando $t \rightarrow 0$.

Demostración. Veáse Temam [27].

Observemos que para resolver numéricamente los ejemplos del capítulo 4 , se utilizan las formulaciones variacionales descritas en (1.11) y (1.19).

\subsection{Condiciones de frontera.}

Si se quiere resolver el problema de Stokes o el problema de Navier-Stokes, se debe especificar una condición de frontera en cada punto de $\Gamma$ para cada variable dependiente. Así, para estudiar el comportamiento de un fluido en 2 o 3 dimensiones se necesitan 2 o 3 condiciones de frontera, respectivamente. Las condiciones más usuales en el caso de fronteras cerradas o acotadas son las condiciones de tipo Dirichlet o de tipo Neumann. En general se usa una condición de tipo Dirichlet sobre la frontera de un sólido, ya que físicamente al usar la hipótesis del continuo se considera que la condición que hay en la interface de un sólido y un fluido es de "no resbalamiento" (no-slip), es decir, si U representa la velocidad de la frontera del sólido, la condición de frontera que se impone sobre la velocidad es

$$
\mathbf{u}=\mathbf{U} \quad \text { sobre la frontera del sólido }
$$

Cuando $\mathbf{U}$ es igual a cero se dice que la condición de frontera es homogénea, en otro caso es no-homogénea.

Por otro lado, se dice que la condición es de tipo Neumann si se prescriben los valores de la derivada normal (exterior) sobre la frontera $\Gamma$. En particular, en mecánica de fluidos o en física la condición de Neumann corresponde a dar una fuerza o un flujo g sobre la frontera $\Gamma$. En el 
caso, de las ecuaciones de Navier-Stokes dicha fuerza está dada por la fuerza de superficie $\mathbf{T} \cdot \mathbf{n}$, donde $\mathbf{n}$ es el vector normal y $\mathbf{T}$ es el tensor de esfuerzos para un fluido Newtoniano definido por la ecuación constitutiva

$$
\mathbf{T}=-p \mathbf{I}+2 \mu \mathrm{D}
$$

con $\mathbf{I}$ un tensor unitario y $\mathbf{D}$ es el tensor de deformación (strain tensor) $\mathbf{D}=\frac{1}{2}\left(\frac{\partial u_{i}}{\partial x_{j}}+\frac{\partial u_{j}}{\partial x_{i}}\right)$.

Para determinar como es la fuerza de superficie $\mathbf{T} \cdot \mathbf{n}$ sobre $\Gamma$, basta con especificar el valor de su componente normal $\left(\mathbf{T}_{n} \equiv^{t} \mathbf{n} \cdot \mathbf{T} \cdot \mathbf{n}\right)$ y de su componente tangencial $\left(\mathbf{T}_{t} \equiv^{t} \mathbf{t} \cdot \mathbf{T} \cdot \mathbf{n}\right)$ sobre $\Gamma$. Por ejemplo, si se desprecia la curvatura de la frontera $\Gamma$ y se considera que $\Omega \subset \mathbb{R}^{2}$, entonces las condiciones de Neumann están dadas por:

$$
\begin{aligned}
& \mathbf{T}_{n}=-p+2 \mu \frac{\partial u_{n}}{\partial n}=g_{1} \\
& \mathbf{T}_{t}=\mu\left(\frac{\partial u_{n}}{\partial t}+\frac{\partial u_{t}}{\partial n}\right)=g_{2}
\end{aligned}
$$

en donde $\mathbf{n}$ es el vector normal, $\mathbf{t}$ es el vector tangente, $u_{n}=\mathbf{u} \cdot \mathbf{n}, u_{t}=\mathbf{u} \cdot \mathbf{t}$ y $\mathbf{g}=\left(g_{1}, g_{2}\right)$. Al vector $\mathbf{T}_{n}$ se le llama condición de esfuerzos normales y a $\mathbf{T}_{t}$ condición de esfuerzos cortantes o tangenciales.

En general, las condiciones tipo Neumann se consideran como condiciones de frontera naturales, es decir, se añaden a la formulación débil, y como no aparecen en los espacios definidos para la velocidad, la presión y las funciones prueba, los nodos sobre $\Gamma$ que esten asociados a condiciones de frontera tipo Neumann se toman como grados de libertad. Por lo cual, es más importante verificar que en el código que se presenta se encuentren bien incorporadas las condiciones de frontera tipo Dirichlet. Debido a esto se escogieron ejemplos que sólo tengan este tipo de condiciones (homogéneas y no homogéneas).

\subsection{Métodos numéricos más comunes para resolver las ecua- ciones de N-S para una formulación velocidad-presión.}

Las formulaciones variacionales que se describieron en el apartado anterior son las más comunes cuando se trabaja con la formulación velocidad-presión. Para cada una de ellas existen varias formas o métodos de resolución. Los métodos más utilizados para resolver numéricamente estos 
problemas son: el método mixto o integrado, los métodos de penalización y el método solenoidal o de divergencia libre. A continuación se explicarán cada uno de los métodos anteriores.

\subsubsection{Método integrado o mixto.}

El método mixto aproxima simultáneamente a la velocidad y a la presión usando el método del elemento finito. Para ello, no es necesario desacoplar estas variables, por lo cual, se requieren de formulaciones tipo velocidad-presión como las formulaciones variacionales descritas en (1.11) y (1.19), además se precisa que el elemento finito sea mixto.

Un aspecto que se consideró en la elección del elemento finito mixto fue que los polinomios de interpolación que aproximan a la presión sean al menos de un grado menor que los polinomios que aproximan a la velocidad. La consideración anterior no es suficiente para la admisibilidad del elemento, por lo que se requiere de una condición de "consistencia" sobre los espacios discretos de la velocidad y de la presión. Ésta es conocida por la condición inf-sup discreta (o condición de Ladyzhenskaya-Babuška-Brezzi discreta) (veánse [3], [9]). Uno de los elementos admisibles más usados son los llamados elementos de Taylor-Hood que están claramente descritos en Cuvelier [7], Ciarlet [5] o Gunzburger [10].

En general las matrices que se obtienen al discretizar los problemas variacionales son ralas (sparse). En caso de que se trabaje con el problema de Stokes, el sistema de ecuaciones que se obtiene es lineal y simétrico. Se resuelve usando algún método directo o método iterativo, su elección se hace dependiendo de la estructura y propiedades de la matriz asociada a dicho sistema. Por otro lado, si se está resolviendo el problema de Navier-Stokes el sistema que se obtiene al discretizarlo es no lineal y se puede resolver directamente usando el método de Newton o los métodos cuasi-Newton, lo que resulta ser muy caro. Otra forma de resolverlo es utilizando métodos iterativos de punto fijo. Por ejemplo, uno de tales métodos consiste en: evaluar el término convectivo con la velocidad calculada en la iteración $n-1$, y obtener la velocidad $\mathbf{u}^{n}$ en la n-ésima iteración por medio de

$$
\begin{gathered}
-\frac{1}{R e} \Delta \mathbf{u}^{n}+\nabla p^{n}=\mathbf{f}-\mathbf{u}^{\mathbf{n}-\mathbf{1}} \cdot \nabla \mathbf{u}^{\mathbf{n}-\mathbf{1}}, \\
-\boldsymbol{\nabla} \cdot \mathbf{u}^{n}=0 .
\end{gathered}
$$

Lo atractivo de este método es que el sistema de ecuaciones lineales solamente se resuelve una 
vez y lo único que se va actualizando en cada iteración es el vector del lado derecho.

La principal ventaja del método mixto es que calcula simultáneamente la velocidad y la presión. Sin embargo, su mayor desventaja es que el sistema de ecuaciones que se forma es muy grande, ya que para cada nodo hay 2,3 o 4 grados de libertad dependiendo si estamos trabajando en $\mathbb{R}, \mathbb{R}^{2}$ o $\mathbb{R}^{3}$, respectivamente. Por lo cual se necesita mucho tiempo de cómputo y bastante espacio de memoria, a pesar de que las matrices sean ralas y se usen métodos de perfil (o métodos skyline) para almacenarlas.

\subsubsection{Métodos de penalización.}

La idea de los métodos de penalización es perturbar la ecuación de continuidad con un pequeño término que contenga a la presión:

$$
\varepsilon p+\nabla \cdot \mathbf{u}=0
$$

donde $\varepsilon$ es un parámetro pequeño. Para exhibir el significado físico de este parámetro se considera la derivada material de la densidad relativa dada por

$$
\frac{1}{\rho} \frac{D \rho}{D t}=-\nabla \cdot \mathbf{u}
$$

(veáse Currie [6]). De la relación anterior y dado que el volumen $\widehat{V}$ es igual a $1 / \rho$, se deduce que

$$
\frac{1}{\widehat{V}} \frac{D \widehat{V}}{D t}=\nabla \cdot \mathbf{u} .
$$

Por otro lado, puesto que el volumen depende de la presión y la temperatura se pueden usar algunas relaciones de la termodinámica bajo condiciones isotérmicas de tal manera que se verifica lo siguiente:

$$
\frac{1}{\widehat{V}} \frac{D \widehat{V}}{D t}=-\gamma M^{2} \frac{D p}{D t}
$$

en donde la constante $\gamma=c_{p} / c_{v}, c_{p}$ y $c_{v}$ son las capacidades caloríficas a presión y volumen constante, respectivamente. Además el número de Mach está definido por $M=U / c$, con $U$ la velocidad característica y $c$ la velocidad del sonido en el fluido. 
De (1.25') y (1.25") se obtiene que

$$
\gamma M^{2} \frac{D p}{D t}+\nabla \cdot \mathbf{u}=0
$$

Observemos que si el número de Mach es pequeño $(U<<c)$ y los cambios de presión son pequeños, entonces el término $\varepsilon p$ de la ecuación (1.25) se puede comparar con el primer término de la ecuación (1.25"'). En tal caso se interpreta a la perturbación $\varepsilon p$ como la "desviación de incompresibilidad del fluido" y se usa para desacoplar la velocidad de la presión. Es decir, si sustituimos (1.25) en (0.2) el problema de Stokes estacionario correspondiente a $(0.4),(0.2)$ se puede escribir como

$$
\begin{aligned}
& \text { Encontrar } \mathbf{u}=\mathbf{u}(\mathbf{x}) \text { y } p=p(\mathbf{x}), \quad \mathbf{x} \in \Omega \cup \Gamma \text { tal que } \\
& -\frac{1}{\operatorname{Re}} \Delta \mathbf{u}+\nabla p=\mathbf{f} \quad \text { en } \Omega, \\
& p=-\frac{1}{\varepsilon} \nabla \cdot \mathbf{u} \quad \text { en } \Omega, \\
& \mathbf{u}=\mathbf{g} \quad \text { sobre } \Gamma \text {. }
\end{aligned}
$$

Luego si reemplazamos la ecuación (1.27) en (1.26), está última quedará en función solamente de la velocidad. Entonces la velocidad y la presión se calculan independientemente, por lo tanto el elemento finito no requiere tener una presión continua. La familia de elementos de Crouzeix-Raviart son los que comúnmente se usan en los métodos de penalización, éstos son más sofisticados que los elementos de Taylor-Hood por la discontinuidad de la presión (veánse Cuvelier [7] o Gunzburger [10]).

El sistema de ecuaciones formado por (1.26)-(1.28) es llamado la formulación de la función de penalización donde $\frac{1}{\varepsilon}$ es el parámetro de penalización. Existen varias formas de resolver el problema (1.26)-(1.28), por ejemplo, existe el método de función de penalización discreto, el método de función de penalización iterativa, etc.. Para ver los detalles de estos métodos se pueden consultar [7], [3] y [10].

La ventaja de los métodos de penalización es que desacoplan la velocidad de la presión, en particular el método de función de penalización discreto es muy eficiente para resolver las ecuaciones de Navier-Stokes, ya que permite que los sistemas de ecuaciones sean más pequeños 
y las matrices asociadas tengan mejor estructura que en los métodos mixtos. De esta manera, se pueden utilizar métodos de perfil para almacenarlas y resolver sin utilizar pivoteo parcial. Estas propiedades hacen que se ahorre tiempo en los cálculos y memoria. La principal desventaja del método de penalización es tener que elegir adecuadamente el parámetro de penalización porque una mala elección puede originar que la matriz sea casi singular.

\subsubsection{Método solenoidal o de divergencia libre.}

El método solenoidal se utiliza con el tipo de formulaciones variacionales (1.14) y (1.21) dadas en los apartados 1.2 y 1.3, respectivamente,en donde el espacio $\mathbf{H}$ div de funciones de prueba contienen la condición $\boldsymbol{\nabla} \cdot \mathbf{u}=0$, por lo cual se les llama funciones base de divergencia libre, pues generan una función discreta $\mathbf{u}_{h}$ que tiene divergencia libre.

Este método consiste en construir funciones base de divergencia libre, que respeten el desacoplamiento de la presión y la velocidad, sin necesidad de introducir un parámetro grande como en el método de penalización. Además, el sistema resultante es más pequeño que el obtenido en el método de penalización.

En la literatura consultada no se encontró un ejemplo en donde se construyan funciones base usando elementos Hood-Taylor que satisfagan la condición de incompresibilidad. Sin embargo, se pueden construir funciones base de divergencia libre a través de elementos tipo CrouzeixRaviart. Los cálculos aparecen en [7], ahí mismo se explica la gran dificultad que existe para problemas en tres dimensiones, ya que los cálculos para construir las funciones base son mucho más sofisticados; ésta es la mayor desventaja de este método.

La principal ventaja del método solenoidal es que requiere de menos tiempo de cálculo y memoria comparados con los otros dos métodos. Otra importante ventaja comparada con el método de penalización, es que no necesita del parámetro $\frac{1}{\epsilon}$, el cual puede causar problemas si $\varepsilon$ es muy pequeño, pero no así con la precisión de la máquina.

En este capítulo se presentaron dos formulaciones débiles tipo velocidad-presión, pero solamente se usarán las formulaciones (1.11) ó (1.19), por ser las necesarias para resolver numéricamente los problemas de Stokes ó de Navier-Stokes, respectivamente, utilizando el método mixto o integrado (veáse apartado 1.5.1) 


\section{Capítulo 2}

\section{Discretización de las ecuaciones de Navier-Stokes vía el método del}

\section{Elemento Finito}

En este trabajo se usa la técnica de Elemento Finito para resolver numéricamente los problemas de Stokes y de Navier-Stokes. Y puesto que se quieren obtener simultáneamente los valores de la velocidad y de la presión, se utiliza el método numérico mixto (veáse apartado 1.5.1), por lo cual únicamente se discretizarán las formulaciones variacionales (1.11)-(1.12) y (1.19)-(1.20) deducidas en los apartados 1.2 y 1.3 , respectivamente.

En el apartado 1.5.1 se mencionó que para aproximar simultáneamente la velocidad y la presión se requiere de elementos finitos que sean mixtos. Esta propiedad la cumplen los elementos del tipo Taylor-Hood, los cuales son muy utilizados en la literatura por su sencillez y por cumplir con la condición de Ladyzhenskaya-Babuška-Brezzi. Por ello se decidió usar en este trabajo el elemento rectangular tipo Taylor-Hood que involucra un campo de velocidades bicuadráticas y un campo de presiones bilineales sobre el elemento (veáse Figura 2-1a).

En este capítulo se presentan las definiciones formales del elemento de referencia tipo TaylorHood, así como los elementos equivalentes-afines y los elementos isoparamétricos (veánse aparta- 
dos 2.1 y 2.2). Además se expone cómo se calculan las componentes y cuál es la estructura de la matriz global, así como de las matrices locales (veáse $2.4,2.5$ y 2.6).

\subsection{Discretización de un dominio poligonal.}

En este apartado se considera que $\Omega$ es un dominio en el plano $\mathbb{R}^{2}$ cuya frontera $\Gamma$ tiene forma poligonal, decimos entonces $\Omega$ es un dominio poligonal.

Para construir una triangulación $\mathcal{T}_{h}$ de la región $\bar{\Omega}$, se divide a $\bar{\Omega}$ en subregiones formadas por cuadriláteros $\Omega_{e}$ que satisfacen las propiedades siguientes:

(i) $\bar{\Omega}=\underset{\Omega_{e} \in \mathcal{T}_{h}}{\cup} \Omega_{e}$

(ii) Cada $\Omega_{e} \in \mathcal{T}_{h}$ es cerrado y su interior $\stackrel{\circ}{\Omega}_{e}$ es no vacío.

(iii) Si $\Omega_{e}$ y $\Omega_{l}$ son dos subregiones distintas de $\mathcal{T}_{h}$, entonces $\stackrel{\circ}{\Omega}_{e} \cap \stackrel{\circ}{\Omega}_{l}=\emptyset$.

(iv) La frontera $\Gamma_{e}$ de cada $\Omega_{e} \in \mathcal{T}_{h}$ es Lipschitz continua.

(v) Cualquier lado de cada cuadrilátero $\Omega_{e} \in \mathcal{T}_{h}$ es un subconjunto de la frontera $\Gamma$ o es un lado de otro cuadrilátero $\Omega_{l} \in \mathcal{T}_{h}$.

Por otro lado, cada cuadrilátero $\Omega_{e}$ está asociado a un par de elementos finitos definido por las tripletas $\left(\Omega_{e}, Q_{2}\left(\Omega_{e}\right), \sum_{e}\right)$ y $\left(\Omega_{e}, Q_{1}\left(\Omega_{e}\right), \sum_{e}^{\prime}\right)$, en donde

$\mathrm{Q}_{1}\left(\Omega_{e}\right)=\left\{q: q(\mathbf{x})=c_{1}+c_{2} x+c_{3} y+c_{4} x y\right.$, en donde las $\left.c_{i} \in \mathbb{R} \quad \mathrm{y}(x, y) \in \Omega_{e}\right\}$

$$
\mathrm{Q}_{2}\left(\Omega_{e}\right)=\left\{v: v(\mathbf{x})=c_{1}+c_{2} x+c_{3} x^{2}+c_{4} y+c_{5} x y+c_{6} x^{2} y+c_{7} y^{2}+c_{8} x y^{2}+c_{9} x^{2} y^{2}\right.
$$
en donde las $c_{i} \in \mathbb{R}$ y $\left.(x, y) \in \Omega_{e}\right\}$,

$\mathrm{Q}_{2}\left(\Omega_{e}\right)=\left[\mathrm{Q}_{2}\left(\Omega_{e}\right)\right]^{2} \cdot \sum_{e} \mathrm{y} \sum_{e}^{\prime}$ son los conjuntos de grados de libertad definidos por $\sum_{e}=\left\{\mathbf{v}\left(\mathbf{a}_{i}\right), i=1, \ldots, 9 \mid \mathbf{v} \in \mathbf{Q}_{2}\left(\Omega_{e}\right)\right.$, donde $\mathbf{a}_{i}$ son las coordenadas de los vértices del cuadrilátero $\Omega_{e}$, los puntos medios de cada uno de sus lados y el punto medio de la subregión $\left.\Omega_{e}\right\}$ 


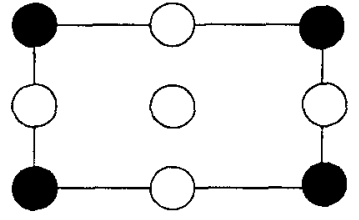

(a)

Nodo para la presión
y la velocidad.
Nodo para la
velocidad.

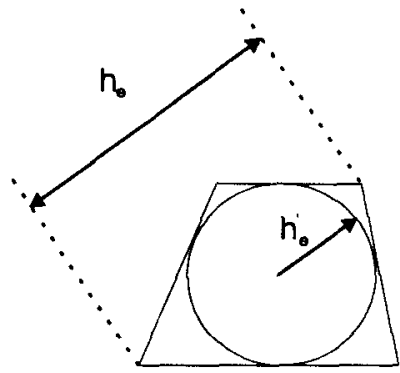

(b) $h$, es el diámetro del elemento e.

h. es el dlámetro del módmo círculo contenido en el elemento e.

Figura 2-1: Elemento rectangular tipo Taylor-Hood y dos parámetros $h_{e} \mathrm{y} h_{e}^{\prime}$.

$\sum_{e}^{\prime}=\left\{q\left(\mathbf{a}_{i}\right), i=1, \ldots, 4 \mid q \in Q_{1}\left(\Omega_{e}\right)\right.$, en donde $\mathbf{a}_{i}$ son las coordenadas de los vértices del cuadrilátero $\left.\Omega_{e}\right\}$. veáse Figura 2-1a.

Cada tripleta es unisolvente, es decir, cada función $\mathbf{v} \in \mathbf{Q}_{2}\left(\Omega_{e}\right)$ y cada función $q \in \mathrm{Q}_{1}\left(\Omega_{e}\right)$ están únicamente determinadas por sus grados de libertad $\sum_{e} \circ \sum_{e}^{\prime}$ correspondientes.

Otra propiedad que satisfacen los cuadriláteros de la triangulación $\mathcal{T}_{h}$ es la siguiente:

(vi) Existe un ángulo $\theta_{0}$ tal que todos los ángulos $\theta$ de cada cuadrilátero cumplen que $0<\theta_{0} \leq \theta$

La propiedad (vi) implica que la familia de elementos asociada a $\mathcal{T}_{h}$ es regular [26], es decir, existe dos constantes $\gamma_{1} \geq 1$ y $0<\gamma_{2}<1$ independientes de $h$ tal que $\frac{h_{e}}{h_{\mathrm{e}}} \leq \gamma_{1}$ y además $\left|\cos \theta_{i e}\right| \leq \gamma_{2}, 1 \leq i \leq 4, \forall e \in \mathcal{T}_{h}, \quad \forall h>0$, en donde $h_{e}$ es el diámetro del elemento $\Omega_{e}$, $h_{e}^{\prime}$ es el supremo de los diámetros de todas las circunferencias contenidas en $\Omega_{e}, \operatorname{los} \theta_{i e}$ con $1 \leq i \leq 4$ son los ángulos interiores de $\Omega_{e}$ y $h=\sup _{\Omega_{e} \in \mathcal{T}_{h}} h_{e}$ (veáse Figura 2-1b).

Observemos que la triangulación $\mathcal{T}_{h}$ tiene un número finito de cuadriláteros, digamos $\mathrm{N}_{e}$, los cuales están asociados a $\mathrm{N}_{e}$ pares de elementos finitos, por lo que abusando de la notación, al par de elementos finitos $\left(\Omega_{e}, \mathrm{Q}_{2}\left(\Omega_{e}\right), \Sigma_{e}\right)$ y $\left(\Omega_{e}, \mathrm{Q}_{1}\left(\Omega_{e}\right), \Sigma_{e}^{\prime}\right)$ se le denotará simplemente 
como el elemento $\Omega_{e}$.

\subsubsection{Elemento de referencia y familia afín.}

Como se verá en el apartado 2.5, para construir las matrices locales es necesario calcular varias integrales sobre cada uno de los elementos $\Omega_{e}$, y puesto que los límites de integración varían dependiendo del elemento que se este integrando, estos cálculos se vuelven muy complicados. Así pues, se utiliza un "elemento típico o característico" (el cual se llama elemento de referencia y se denota por $\Omega_{\hat{e}}$ ) para poder calcular la integral sobre $\Omega_{\hat{e}}$ y vía una transformación $\mathbf{T}_{e}$ se recupere el valor de la integral sobre $\Omega_{e}$.

En particular, el elemento de referencia $\Omega_{\hat{e}}$ tipo Taylor-Hood que se utiliza en este trabajo está definido por el par de tripletas $\left(\Omega_{\hat{e}}, \hat{\mathbf{Q}}_{2}\left(\Omega_{\hat{e}}\right), \hat{\Sigma}_{\hat{e}}\right)$ y $\left(\Omega_{\hat{e}}, \hat{\mathrm{Q}}_{1}\left(\Omega_{\hat{e}}\right), \hat{\Sigma}_{\hat{e}}^{\prime}\right)$, en donde $\Omega_{\hat{e}}$ es un cuadrilátero en el plano $(\xi, \eta)$ con vértices en $\hat{\mathbf{a}}_{1}=(-1,-1), \hat{\mathbf{a}}_{2}=(1,-1), \hat{\mathbf{a}}_{3}=(1,1)$ y $\hat{\mathbf{a}}_{4}=(-1,1)$,

$$
\begin{aligned}
\hat{\mathrm{Q}}_{1}\left(\Omega_{\hat{e}}\right)= & \left\{\hat{q}: \hat{q}(\xi, \eta)=c_{1}+c_{2} \xi+c_{3} \eta+c_{4} \xi \eta, \text { donde las } c_{i} \in \mathbb{R} \text { y }(\xi, \eta) \in \Omega_{\hat{e}}\right\}, \\
\hat{\mathrm{Q}}_{2}\left(\Omega_{\hat{e}}\right)= & \left\{\hat{v}: \hat{v}(\xi, \eta)=c_{1}+c_{2} \xi+c_{3} \xi^{2}+c_{4} \eta+c_{5} \xi \eta+c_{6} \xi^{2} \eta+c_{7} \eta^{2}+c_{8} \xi \eta^{2}+c_{9} \xi^{2} \eta^{2},\right. \\
& \text { donde las } \left.c_{i} \in \mathbb{R} \text { y }(\xi, \eta) \in \Omega_{\hat{e}}\right\}
\end{aligned}
$$

y $\quad \hat{\mathbf{Q}}_{2}\left(\Omega_{\hat{e}}\right)=\left[\hat{\mathrm{Q}}_{2}\left(\Omega_{\hat{e}}\right)\right]^{2}$. Los conjuntos de grados de libertad $\hat{\sum}_{\hat{e}} \mathrm{y} \hat{\sum}_{\hat{e}}^{\prime}$ están definidos como

$$
\hat{\sum}_{\hat{e}}=\left\{\hat{\mathbf{v}}\left(\hat{\mathbf{a}}_{i}\right), i=1, \ldots, 9 \mid \hat{\mathbf{v}} \in \hat{\mathbf{Q}}_{2}\left(\Omega_{\hat{e}}\right)\right\} \quad \text { y } \quad \hat{\sum}_{\hat{e}}^{\prime}=\left\{\hat{q}\left(\hat{\mathbf{a}}_{i}\right), i=1, \ldots, 4 \mid \hat{q} \in \hat{\mathrm{Q}}_{1}\left(\Omega_{\hat{e}}\right)\right\}
$$

donde $\hat{\mathbf{a}}_{5}=(0,-1), \hat{\mathbf{a}}_{6}=(1,0), \hat{\mathbf{a}}_{7}=(0,1), \hat{\mathbf{a}}_{8}=(-1,0), \mathrm{y} \hat{\mathbf{a}}_{9}=(0,0)$ (veáse Figura 2-2).

Una base local para el espacio $\hat{Q}_{1}\left(\Omega_{\hat{e}}\right)$ está dada por:

$$
\begin{aligned}
& \widehat{\varphi}_{1}(\xi, \eta)=\frac{1}{4}(1-\xi)(1-\eta), \quad \widehat{\varphi}_{2}(\xi, \eta)=\frac{1}{4}(1+\xi)(1-\eta), \\
& \widehat{\varphi}_{3}(\xi, \eta)=\frac{1}{4}(1+\xi)(1+\eta) \quad \text { y } \quad \widehat{\varphi}_{4}(\xi, \eta)=\frac{1}{4}(1-\xi)(1+\eta) .
\end{aligned}
$$

Mientras que, las funciones base locales ( llamadas también funciones de forma) para $\hat{Q}_{2}\left(\Omega_{\hat{e}}\right)$ 


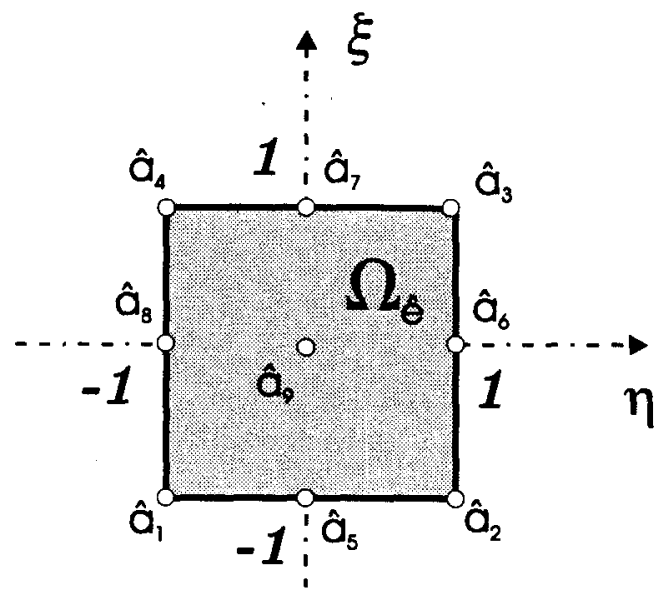

Figura 2-2: Elemento de referencia.

están dadas por:

$$
\begin{array}{ll}
\hat{\psi}_{1}(\xi, \eta)=\frac{1}{4}\left(\xi^{2}-\xi\right)\left(\eta^{2}-\eta\right), & \hat{\psi}_{2}(\xi, \eta)=\frac{1}{4}\left(\xi^{2}+\xi\right)\left(\eta^{2}-\eta\right), \\
\hat{\psi}_{3}(\xi, \eta)=\frac{1}{4}\left(\xi^{2}+\xi\right)\left(\eta^{2}+\eta\right), & \hat{\psi}_{4}(\xi, \eta)=\frac{1}{4}\left(\xi^{2}-\xi\right)\left(\eta^{2}+\eta\right), \\
\hat{\psi}_{5}(\xi, \eta)=\frac{1}{2}\left(1-\xi^{2}\right)\left(\eta^{2}-\eta\right), & \hat{\psi}_{6}(\xi, \eta)=\frac{1}{2}\left(\xi^{2}+\xi\right)\left(1-\eta^{2}\right), \\
\hat{\psi}_{7}(\xi, \eta)=\frac{1}{2}\left(1-\xi^{2}\right)\left(\eta^{2}+\eta\right), & \hat{\psi}_{8}(\xi, \eta)=\frac{1}{2}\left(\xi^{2}-\xi\right)\left(1-\eta^{2}\right) \quad \mathrm{y} \\
\hat{\psi}_{9}(\xi, \eta)=\left(1-\xi^{2}\right)\left(1-\eta^{2}\right) . &
\end{array}
$$

Se sabe que una aplicación afín transforma rectas en rectas, por lo cual, esta transformación es un buen candidato para relacionar al elemento de referencia $\Omega_{\tilde{e}}$ con cada elemento $\Omega_{e}$ contenido en el dominio poligonal $\bar{\Omega}$, es decir, cada elemento $\Omega_{e} \in \mathcal{T}_{h}$ tiene asociada una transformación afín $\mathbf{T}_{e}: \Omega_{\hat{e}} \rightarrow \Omega_{e}$ definida por:

$$
\mathbf{T}_{e}(\widehat{\mathbf{x}})=\mathbf{B}_{e} \widehat{\mathbf{x}}+\mathbf{b}_{e}, \quad \text { en donde } \quad \widehat{\mathbf{x}}=(\xi, \eta) \in \Omega_{\hat{e}} .
$$

Diremos que dos elementos finitos $\Omega_{\hat{e}}$ y $\Omega_{e}$ son equivalentes-afines si existe una transformación afín definida por (2.3) invertible tal que cumpla con las condiciones siguientes:

$$
\Omega_{e}=\mathbf{T}_{e}\left(\Omega_{\hat{e}}\right)
$$




$$
\begin{aligned}
& \mathrm{Q}_{1}\left(\Omega_{e}\right)=\left\{q: \Omega_{e} \rightarrow \mathbb{R} ; \quad q=\hat{q} \circ \mathbf{T}_{e}^{-1}, \quad \hat{q} \in \hat{\mathrm{Q}}_{1}\left(\Omega_{\hat{e}}\right)\right\} \\
& \mathrm{Q}_{2}\left(\Omega_{e}\right)=\left\{v: \Omega_{e} \rightarrow \mathbb{R} ; \quad v=\hat{v} \circ \mathbf{T}_{e}^{-1}, \quad \hat{v} \in \hat{\mathrm{Q}}_{2}\left(\Omega_{\hat{e}}\right)\right\} \\
& \quad \mathbf{a}_{i}=\mathbf{T}_{e}\left(\hat{\mathbf{a}}_{i}\right), \quad \text { con } i=1,2, \ldots, 9 .
\end{aligned}
$$

Una familia de elementos finitos se dice que es una familia afín si todos sus elementos son equivalentes-afines al mismo elemento $\Omega_{\hat{e}}$ (por eso se llama elemento de referencia). Por lo tanto, una forma de trabajar dominios poligonales es usando una familia afin de elementos finitos.

En el apartado 2.2.1 se da un ejemplo de cómo se calculan las componentes de la matriz $\mathbf{B}_{e}$ y del vector $b_{e}$, además en la sección 2.6 se muestra cómo se usa la transformación afín para evaluar las matrices locales.

\subsection{Discretización de un dominio curvo.}

En este apartado se considera que $\Omega$ es un dominio en el plano $\mathbb{R}^{2}$ cuya frontera $\Gamma$ tiene forma curva, en este caso diremos que $\Omega$ es un dominio curvo.

Una forma de trabajar dominios curvos es utilizando una triangulación cuyos elementos finitos tengan lados curvos, esta característica la tienen los elementos isoparamétricos que se describen a continuación.

\subsubsection{Elementos y transformación isoparamétricos.}

En la Figura 2-3 se observa que la malla está formada por cuadriláteros que tienen dos lados curvos. Definiremos este tipo de elementos curvos en forma análoga a lo que se hizo con los elementos equivalentes-afines. Por ello, el elemento de referencia $\Omega_{\hat{e}}$ que usaremos es el mismo que el definido en el apartado 2.1.1.

Para dar una idea intuitiva de lo que hace una transformación isoparamétrica y cómo se forma un elemento isoparamétrico vamos a suponer que queremos que el lado que pasa a través de los puntos $\hat{\mathbf{a}}_{2}, \hat{\mathbf{a}}_{6} \mathrm{y} \hat{\mathbf{a}}_{3}$ del elemento de referencia $\Omega_{\hat{e}}$ dado en la Fig. 2-4 mediante una transformación $\mathbf{T}_{e}$ sea deformado a una parábola, con la restricción de que $\mathbf{T}_{e}\left(\hat{\mathbf{a}}_{2}\right)=\mathbf{a}_{2}$, $\mathbf{T}_{e}\left(\hat{\mathbf{a}}_{6}\right)=\mathbf{a}_{6} \mathrm{y} \mathbf{T}_{e}\left(\hat{\mathbf{a}}_{3}\right)=\mathbf{a}_{3}$. Esto se puede obtener al interpolar los puntos $\mathbf{a}_{2}, \mathbf{a}_{6} \mathrm{y} \mathbf{a}_{3}$ usando 


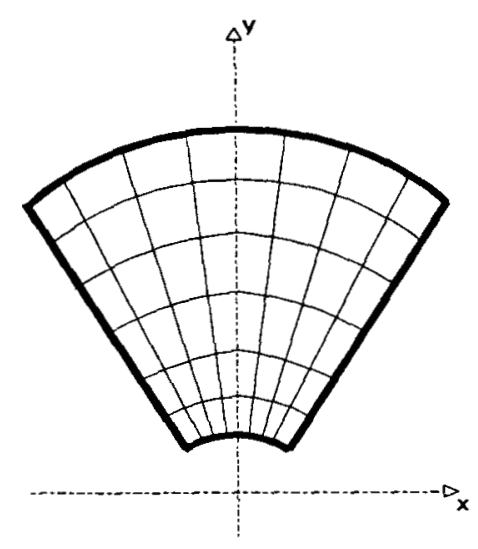

Figura 2-3: Malla.

los polinomios de interpolación de Lagrange cuadráticos dados en (2.2). Si se hace lo mismo para cada uno de los lados de $\Omega_{\hat{e}}$ se obtendrá un elemento "rectangular" con lados curvos. Observemos que si los puntos $\mathbf{a}_{2}, \mathbf{a}_{6} \mathbf{y} \mathbf{a}_{3}$ son colineales el lado de $\Omega_{\hat{e}}$ se reduce a una recta. Supongamos ahora que identificamos $\mathrm{N}_{T}$ nodos de los nueve que hay en el elemento de referencia $\Omega_{\hat{e}}$, entonces para cada elemento $\Omega_{e}$ definimos una transformación $\mathbf{T}_{e}: \Omega_{\hat{e}} \rightarrow \Omega_{e}$ dada por:

$$
\begin{gathered}
\mathbf{T}_{e}(\xi, \eta)=(x, y), \quad \text { en donde } \\
x=\sum_{j=1}^{N_{T}} x_{j} \hat{\psi}_{j}(\xi, \eta), \quad y=\sum_{j=1}^{N_{T}} y_{j} \hat{\psi}_{j}(\xi, \eta)
\end{gathered}
$$

en donde las $\left(x_{j}, y_{j}\right)$ son las $x-y$ coordenadas de los correspondientes $\mathrm{N}_{T}$ puntos nodales del elemento $\Omega_{e}$, y las $\hat{\psi}_{j}(\xi, \eta)$ son las funciones base locales de $\hat{\mathrm{Q}}_{2}\left(\Omega_{\hat{e}}\right)$, definidas en las ecuaciones $(2.2)$.

Si denotamos por $\hat{\mathrm{P}}_{k}\left(\Omega_{\hat{e}}\right)$ al conjunto formado por los polinomios de grado menor o igual que $k$ sobre $\Omega_{\hat{e}} \in \mathbb{R}^{n}$, entonces en caso de que cada componente de la transformación $\mathbf{T}_{e}$ pertenezca al espacio $\hat{\mathrm{Q}}_{1}\left(\Omega_{\hat{e}}\right)$ (o $\hat{\mathrm{P}}_{1}\left(\Omega_{\hat{e}}\right)$ ), se dice que la transformación es subparamétrica y abusando de la notación escribiremos $\mathbf{T}_{e} \in \hat{\mathbf{Q}}_{1}\left(\Omega_{\hat{e}}\right)$. Si las componentes de $\mathbf{T}_{\boldsymbol{e}}$ pertenecen al espacio $\hat{\mathbf{Q}}_{2}\left(\Omega_{\hat{e}}\right)$, se dice que $\mathbf{T}_{e}$ es una transformación isoparamétrica $\left(\mathbf{T}_{e} \in \hat{\mathbf{Q}}_{2}\left(\Omega_{\hat{e}}\right)\right)$. Es decir si $\mathrm{N}_{T}<9$ la transformación es subparamétrica y si $\mathrm{N}_{T}=9$ la transformación es isoparamétrica ( veáse Figura 2-4). 

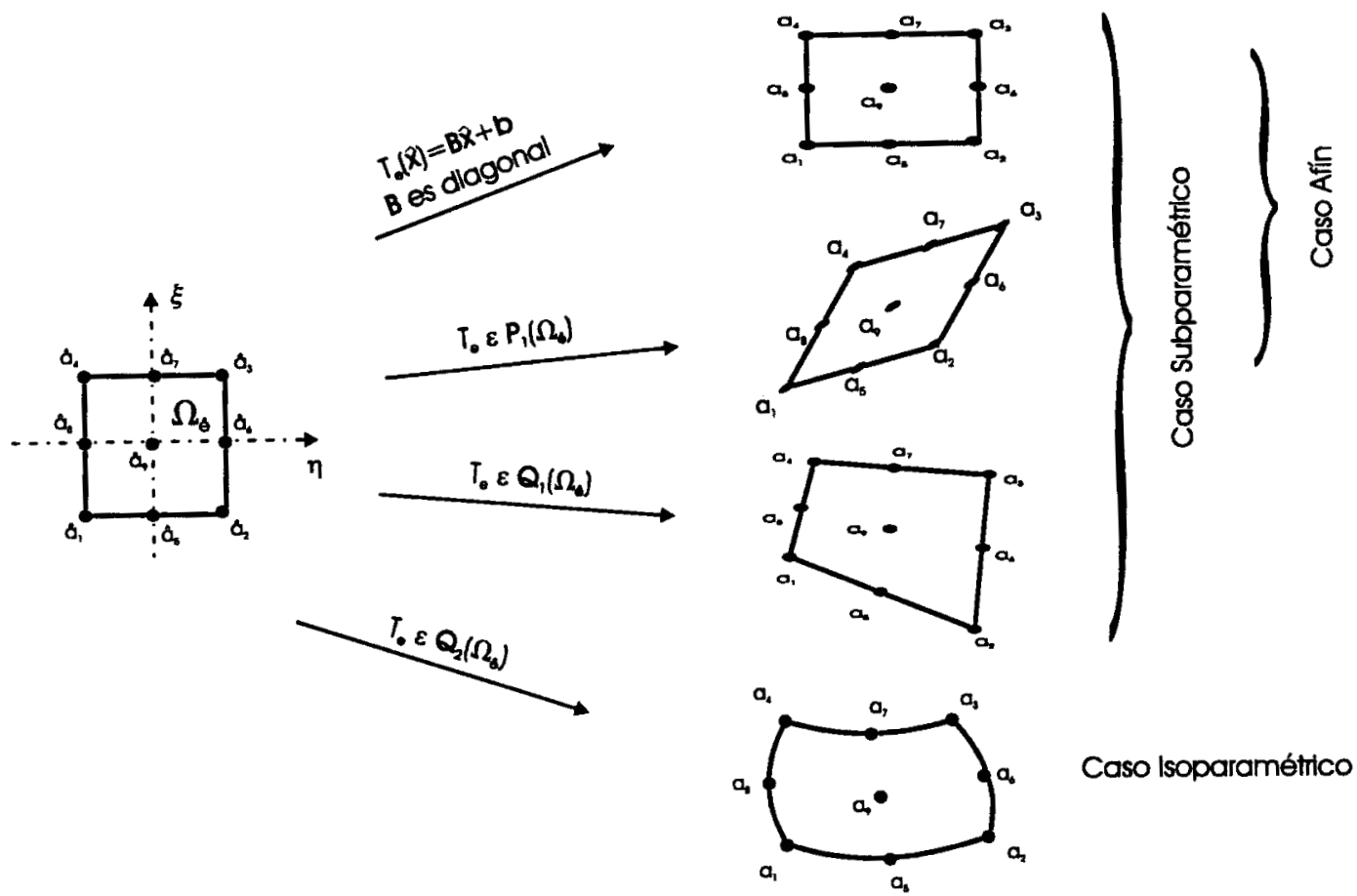

Figura 2-4: Diderentes tipos de cuadriláteros.

En particular una transformación isoparamétrica cumple con las condiciones (2.4)-(2.7), por lo cual la transformación $\mathbf{T}_{e}$ está únicamente determinada por los puntos $\mathbf{a}_{i}=\left(x_{i}, y_{i}\right)$ con $i=1, \ldots, 9$, ya que $\mathbf{T}_{e}$ satisface la propiedad (2.7) .También $\mathbf{T}_{e}$ es uno a uno si $h$ es suficientemente pequeña [5] e invertible si se satisface la propiedad (vi) (veáse [20]) . Luego, se define a un elemento isoparamétrico como la tripleta $\left(\Omega_{e}, \mathbf{Q}_{2}\left(\Omega_{e}\right), \sum_{e}\right)$, en donde

$$
\begin{aligned}
& \mathbf{Q}_{2}\left(\Omega_{e}\right)=\left\{\mathrm{v}: \mathbf{v}(\mathbf{a})=\hat{\mathbf{v}}\left(\mathbf{T}_{e}^{-1}(\mathbf{a})\right), \mathbf{a} \in \Omega_{e}, \quad \hat{\mathbf{v}} \in \hat{\mathbf{Q}}_{2}\left(\Omega_{\hat{e}}\right)\right\} \quad \mathrm{y} \\
& \sum_{e}=\left\{\mathbf{v}\left(\mathbf{a}_{i}\right): \mathbf{a}_{i}=T_{e}\left(\hat{\mathbf{a}}_{i}\right), i=1, \ldots, 9\right\}
\end{aligned}
$$

(veánse las Figuras 2-4 y 2-5).

Denotaremos por $\Omega_{h}$ a la unión de elementos isoparamétricos, es decir, $\Omega_{h}=\underset{\Omega_{e} \in \mathcal{T}_{h}}{\cup} \mathbf{T}_{e}\left(\Omega_{\hat{e}}\right)$. Esta familia de elementos isoparamétricos es regular si:

(i) La familia afín $\mathcal{T}_{h}$ asociada a la familia isoparamétrica $\boldsymbol{\Omega}_{h}$ es regular (veáse apartado 2.1),

(ii) la frontera $\Gamma$ de la región $\Omega$ es suficientemente suave $y$

(iii) si para cada $\Omega_{e} \in \Omega_{h}$ los nodos $\tilde{a}_{i}$, con $i=1, \ldots, 9$ de su elemento equivalente-afín 


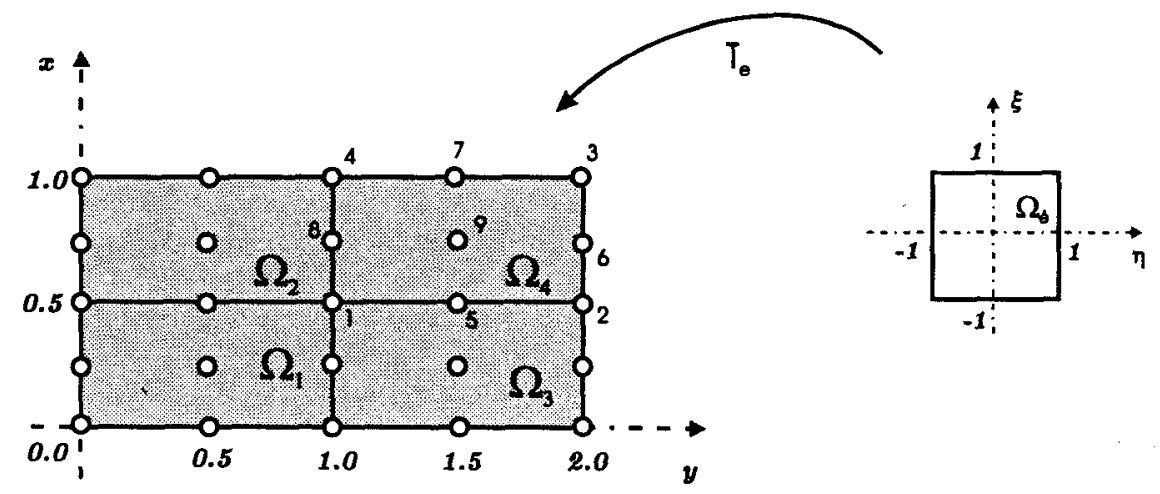

Figura 2-5: Malla formada por cuatro elementos.

asociado $\widetilde{\Omega}_{e} \in \mathcal{T}_{h}$, cumple que:

$$
\begin{aligned}
\widetilde{a}_{i} & =a_{i} \quad \text { para } i=1,2,3,4 \quad \text { y } \\
\left\|\widetilde{a}_{i}-a_{i}\right\| & =O\left(h^{2}\right) \quad \text { para } i=5, \ldots, 9,
\end{aligned}
$$

donde las $a_{i}$ son los nodos correspondientes al elemento isoparamétrico $\Omega_{e}$ (veánse Ciarlet [5], Johnson [15] y Stenberg [26]).

En la Figura 2-4 se observa que las transformaciones afines son un caso particular de las transformaciones subparamétricas, para mostrar esto y ejemplificar cómo se obtiene la matriz $\mathbf{B}_{e}$ y el vector $\mathbf{b}_{e}$ de una transformación afín, se considera el elemento de referencia y la malla descrita en la Figura 2-5.

Supongamos que $\mathbf{T}_{4} \in \hat{\mathbf{Q}}_{1}\left(\Omega_{\hat{e}}\right)$, entonces la transformación $\mathbf{T}_{4}$ asociada al elemento 4 está definida por:

$$
\mathbf{T}_{4}(\xi, \eta)=\left(\begin{array}{c}
\widehat{\varphi}_{1}(\xi, \eta)+2 \widehat{\varphi}_{2}(\xi, \eta)+2 \widehat{\varphi}_{3}(\xi, \eta)+\widehat{\varphi}_{4}(\xi, \eta) \\
\frac{1}{2} \widehat{\varphi}_{1}(\xi, \eta)+\frac{1}{2} \widehat{\varphi}_{2}(\xi, \eta)+\widehat{\varphi}_{3}(\xi, \eta)+\widehat{\varphi}_{4}(\xi, \eta)
\end{array}\right)
$$

en donde, sustituyendo las expresiones para las $\widehat{\varphi}_{i}(\xi, \eta)$ que están definidas en $(2.1)$ y haciendo algunos cálculos sencillos se obtiene la expresión siguiente: 


$$
\mathbf{T}_{4}(\xi, \eta)=\left(\begin{array}{cc}
\frac{1}{2} & 0 \\
0 & \frac{1}{4}
\end{array}\right)\left(\begin{array}{c}
\xi \\
\eta
\end{array}\right)+\left(\begin{array}{c}
\frac{3}{2} \\
\frac{3}{4}
\end{array}\right)
$$

Luego, esta última expresión toma la forma de la ecuación (2.3) con la matriz $\mathbf{B}_{4}$ y el vector $\mathbf{b}_{4}$ definidos por

$$
\mathbf{B}_{4}=\left(\begin{array}{cc}
\frac{1}{2} & 0 \\
0 & \frac{1}{4}
\end{array}\right) \quad y \quad \mathbf{b}_{4}=\left(\begin{array}{c}
\frac{3}{2} \\
\frac{3}{4}
\end{array}\right) .
$$

Ahora, para ejemplificar que las transformaciones afines son un caso degenerado de las transformaciones isoparamétricas se utiliza el mismo elemento 4 . Es decir, si $\mathbf{T}_{\mathbf{4}} \in \hat{\mathbf{Q}}_{2}\left(\Omega_{\hat{e}}\right)$, la transformación isoparamétrica asociada al elemento 4 está dada por

$$
\mathbf{T}_{4}(\xi, \eta)=\left(\begin{array}{c}
\hat{\psi}_{1}+2 \hat{\psi}_{2}+2 \hat{\psi}_{3}+\hat{\psi}_{4}+\frac{3}{2} \hat{\psi}_{5}+2 \hat{\psi}_{6}+\frac{3}{2} \hat{\psi}_{7}+\hat{\psi}_{8}+\frac{3}{2} \hat{\psi}_{9} \\
\frac{1}{2} \hat{\psi}_{1}+\frac{1}{2} \hat{\psi}_{2}+\hat{\psi}_{3}+\hat{\psi}_{4}+\frac{1}{2} \hat{\psi}_{5}+\frac{3}{2} \hat{\psi}_{6}+\hat{\psi}_{7}+\frac{3}{2} \hat{\psi}_{8}+\frac{3}{2} \hat{\psi}_{9}
\end{array}\right)
$$

Usando las definiciones de las $\hat{\psi}_{i}(\xi, \eta)$ dadas en (2.2) y realizando los cálculos indicados en la igualdad anterior se tiene que

$$
\mathbf{T}_{4}(\xi, \eta)=\left(\begin{array}{cc}
\frac{1}{2} & 0 \\
0 & \frac{1}{4}
\end{array}\right)\left(\begin{array}{c}
\xi \\
\eta
\end{array}\right)+\left(\begin{array}{c}
\frac{3}{2} \\
\frac{3}{4}
\end{array}\right)
$$

que es lo que se esperaba, ya que $\hat{\mathbf{Q}}_{1}\left(\Omega_{\hat{e}}\right) \subset \hat{\mathbf{Q}}_{2}\left(\Omega_{\hat{e}}\right)$. Por lo tanto, los dominios poligonales también se pueden calcular a través de transformaciones isoparamétricas, por ello en este trabajo sólo se ocupan dichas transformaciones.

\subsubsection{Discretización de la velocidad y de la presión.}

Para aproximar las funciones en los espacios de soluciones $H^{1}(\Omega), H_{0}^{1}(\Omega)$ y $L_{0}^{2}(\Omega)$ de las formulaciones débiles (1.11) o (1.19) se usa la familia de elementos isoparamétricos $\Omega_{h}$. De esta manera se obtienen los siguientes espacios discretos: 


$$
\begin{aligned}
& \mathbf{V}_{h}=\left\{\mathbf{v}_{h} \in \mathbf{C}^{0}(\bar{\Omega})\left|\mathbf{v}_{h}\right|_{\Omega_{e}} \in \mathbf{Q}_{2}\left(\Omega_{e}\right), \Omega_{e} \in \Omega_{h}\right\} \\
& \mathbf{V}_{h}^{0}=\left\{\mathbf{v}_{h} \in \mathbf{V}_{h} \mid \quad \mathbf{v}_{h}=0 \text { sobre } \Gamma_{h}\right\}, \\
& Q_{h}^{0}=\left\{q_{h} \in C^{0}(\Omega)\left|q_{h}\right|_{\Omega_{e}} \in \mathrm{Q}_{1}\left(\Omega_{e}\right), \Omega_{e} \in \Omega_{h} ; \quad \int_{\Omega_{h}} q_{h} d \Omega=0\right\},
\end{aligned}
$$

en donde $\mathrm{Q}_{2}\left(\Omega_{e}\right)$ está definido en $(2.9), \Gamma_{h}$ es la frontera de $\Omega_{h}$ y

$$
\mathrm{Q}_{1}\left(\Omega_{e}\right)=\left\{q: q(\mathbf{a})=\hat{q}\left(\mathbf{T}_{e}^{-1}(\mathbf{a})\right), \mathbf{a} \in \Omega_{e}, \quad \hat{q} \in \hat{\mathbf{Q}}_{1}\left(\Omega_{\hat{e}}\right)\right\} \text {. }
$$

Como los elementos $\Omega_{e} \in \Omega_{h} \subset \Omega$ satisfacen la propiedad (iv) y las funciones base de $\mathbf{V}_{h}$ son continuamente diferenciales sobre cada elemento $\Omega_{e}$ y continuas sobre todo el dominio $\Omega_{h}$, además de que las funciones base de $Q_{h}^{0}$ son continuas sobre cada elemento $\Omega_{e}$ e integrables sobre $\Omega_{h}$, se cumple que $\mathbf{V}_{h} \subset \mathbf{H}^{1}(\Omega), \mathbf{V}_{h}^{0} \subset \mathbf{H}_{0}^{1}(\Omega)$ y $Q_{h}^{0} \subset L_{0}^{2}(\Omega)$. En este caso se dice que los espacios $\mathbf{V}_{h}, \mathbf{V}_{h}^{0}$ y $Q_{h}^{0}$ son conformes, siempre que $\Omega_{h} \subseteq \bar{\Omega}$ (veánse Cuvelier [7] y Ciarlet [5]).

\subsection{Discretización de los problemas de Stokes y N-S.}

Para obtener soluciones aproximadas de los problemas (1.11) y (1.19) se tienen que discretizar dichos problemas (recordemos que aún estamos considerando condiciones a la frontera de tipo Dirichlet homogéneas). Para ello, primero daremos la discretización de las variables espaciales empleando los espacios discretos definidos en (2.11). Una formulación débil del problema de Stokes discreto asociado a la familia de elementos isoparamétricos se enuncia como sigue:

$$
\begin{gathered}
\text { Encontrar }\left(\mathbf{u}_{h}, p_{h}\right) \in \mathbf{V}_{h}^{0} \times Q_{h}^{0} \text { tal que } \\
\frac{d}{d t}\left(\mathbf{u}_{h}, \mathbf{v}_{h}\right)+a\left(\mathbf{u}_{h}, \mathbf{v}_{h}\right)+b\left(p_{h}, \mathbf{v}_{h}\right)=L_{f}\left(\mathbf{v}_{h}\right) \quad \forall \mathbf{v}_{h} \in \mathbf{V}_{h}^{0} \\
b\left(q_{h}, \mathbf{u}_{h}\right)=0 \quad \forall q_{h} \in Q_{h}^{0}
\end{gathered}
$$

Ahora para discretizar la variable temporal dividimos el intervalo $[0, T]$ en subintervalos igualmente espaciado de longitud $\Delta t$ y usamos un esquema de Euler hacia atrás implícito, de tal manera que una formulación débil del problema discreto (2.12) es: 
Asumiendo que $\left(\mathbf{u}_{h}^{0}, p_{h}^{0}\right), \ldots,\left(\mathbf{u}_{h}^{\mathrm{N}-1}, p_{h}^{\mathrm{N}-1}\right)$ en $\mathbf{V}_{h}^{0} \times Q_{h}^{0}$ son conocidas, encontrar

$$
\begin{gathered}
\left(\mathbf{u}_{h}^{\mathrm{N}}, p_{h}^{\mathrm{N}}\right) \in \mathbf{V}_{h}^{0} \times Q_{h}^{0} \quad \text { tal que } \\
\begin{array}{c}
\left(\frac{\mathbf{u}_{h}^{\mathrm{N}}-\mathbf{u}_{h}^{\mathrm{N}-1}}{\Delta t}, \mathbf{v}_{h}\right)+a\left(\mathbf{u}_{h}^{\mathrm{N}}, \mathbf{v}_{h}\right)+b\left(p_{h}^{\mathrm{N}}, \mathbf{v}_{h}\right)=L_{f^{\mathrm{N}}}\left(\mathbf{v}_{h}\right) \quad \forall \mathbf{v}_{h} \in \mathbf{V}_{h}^{0}, \\
b\left(q_{h}, \mathbf{u}_{h}^{\mathbb{N}}\right)=0 \quad \forall q_{h} \in Q_{h}^{0} .
\end{array}
\end{gathered}
$$

Dado que la fuerza volumétrica $\mathbf{f}$ en general depende de $\mathbf{x} \mathrm{y}$ de $t$, entonces el operador lineal $L_{f}$ depende del tiempo y se define como $L_{f^{\mathbb{N}}}\left(\mathbf{v}_{h}\right)=L_{f}\left(\mathbf{v}_{h}\right)$.

Análogamente, la discretización completa de la formulación débil del problema de NavierStokes (ecs. (1.19)) es:

$$
\begin{gathered}
\text { Asumiendo que }\left(\mathbf{u}_{h}^{0}, p_{h}^{0}\right), \ldots,\left(\mathbf{u}_{h}^{\mathrm{N}-1}, p_{h}^{\mathrm{N}-1}\right) \text { en } \mathbf{V}_{h}^{0} \times Q_{h}^{0} \text { son conocidas, encontrar } \\
\left(\mathbf{u}_{h}^{\mathrm{N}}, p_{h}^{\mathbb{N}}\right) \in \mathbf{V}_{h}^{0} \times Q_{h}^{0} \quad \text { tal que } \\
\left(\frac{\mathbf{u}_{h}^{\mathbb{N}}-\mathbf{u}_{h}^{\mathrm{N}-1}}{\Delta t}, \mathbf{v}_{h}\right)+a_{1}\left(\mathbf{u}_{h}^{\mathbb{N}} ; \mathbf{u}_{h}^{\mathbb{N}}, \mathbf{v}_{h}\right)+a\left(\mathbf{u}_{h}^{\mathbb{N}}, \mathbf{v}_{h}\right)+b\left(p_{h}^{\mathbb{N}}, \mathbf{v}_{h}\right)=L_{f^{\mathbb{N}}}\left(\mathbf{v}_{h}\right) \quad \forall \mathbf{v}_{h} \in \mathbf{V}_{h}^{0} \\
b\left(q_{h}, \mathbf{u}_{h}^{\mathbb{N}}\right)=0 \quad \forall q_{h} \in Q_{h}^{0} .
\end{gathered}
$$

Las condiciones para la convergencia y estabilidad de este esquema se dan en el siguiente capítulo.

Con respecto a la existencia y unicidad de la solución de la "formulación discreta" (2.13), para el caso de un dominio poligonal, acotado y convexo, se tiene en el teorema siguiente:

Teorema 2.1. Si se satisfacen las condiciones siguientes:

(i) Existe un operador lineal $r_{h} \in \mathfrak{L}\left(\mathbf{H}^{2}(\Omega) ; \mathbf{V}_{h}\right) \cap \mathfrak{L}\left(\left(\mathbf{H}^{2}(\Omega) \cap \mathbf{H}_{0}^{1}(\Omega)\right) ; \mathbf{V}_{h}^{0}\right)$ y un entero $\ell$ tal que

$$
\left\|\mathbf{v}-r_{h} \mathbf{v}\right\|_{1} \leq C h^{m}\|\mathbf{v}\|_{m+1} \quad \forall \mathbf{v} \in \mathbf{H}^{m+1}, \quad 1 \leq m \leq \ell
$$

(ii) Existe un operador lineal $S_{h} \in \mathfrak{L}\left(L^{2}(\Omega) ; Q_{h}\right)$ tal que

$$
\left\|q-S_{h} q\right\|_{0} \leq C h^{m}\|q\|_{m} \quad \forall q \in H^{m}, \quad 1 \leq m \leq \ell
$$

(iii) (Condicióninf-sup discreta) Existe una constante $0<\beta=1 / C$, independiente de la $h$, 
tal que se cumple la relación siguiente

$$
\sup _{\mathbf{0} \neq \mathbf{v}_{h} \in \mathbf{V}_{h}^{0}}\left(\frac{b\left(\mathbf{v}_{h}, q_{h}\right)}{\left|\mathbf{v}_{h}\right|_{1}}\right) \geq \beta\left\|q_{h}\right\|_{0}, \quad \forall q_{h} \in Q_{h}^{0}
$$

entonces el problema (2.13) admite una única solución $\left(\mathbf{u}_{h}, p_{h}\right) \in \mathbf{V}_{h}^{0} \times Q_{h}^{0}$ que tiende a la solución $(\mathbf{u}, p)$ del problema (1.15), es decir,

$$
\lim _{h \rightarrow 0}\left\{\left|\mathbf{u}-\mathbf{u}_{h}\right|_{1}+\left\|q-q_{h}\right\|_{0}\right\}=0
$$

Más aún, cuando $(\mathbf{u}, p)$ pertenece a $\mathbf{H}^{m+1}(\Omega) \times\left(H^{m}(\Omega) \cap L_{0}^{2}(\Omega)\right)$ para algún entero $m$ con $1 \leq m \leq \ell$, la cota de error tiene la forma siguiente:

$$
\left|\mathbf{u}-\mathbf{u}_{h}\right|_{1}+\left\|q-q_{h}\right\|_{0} \leq C_{1} h^{m}\left\{\|\mathbf{u}\|_{m+1}+\|q\|_{m}\right\}
$$

Si $\Omega$ es un dominio poligonal, acotado y convexo la cota de error está dada por:

$$
\left\|\mathbf{u}-\mathbf{u}_{h}\right\|_{0, \Omega} \leq C_{2} h^{m+1}\left\{\|\mathbf{u}\|_{m+1}+\|q\|_{m, \Omega}\right\}
$$

Demostración. En general la demostración se hace de manera similar que la prueba del teorema 1.1, además de probar algunos resultados sobre cotas de error como se ve en Girault y Raviart [9].

Análogamente, en Girault y Raviart [9] se enuncia la existencia y unicidad de la solución de la formulación discreta de Navier-Stokes en un nivel de tiempo fijo y para un dominio poligonal, acotado y convexo.

Se debe mencionar que en particular, las condiciones $(i)-(i i i)$ se satisfacen para una familia de elementos isoparamétricos que sea regular, ya que Stenberg [26] muestra que si

$$
\left\|\widetilde{\boldsymbol{a}}_{i}-a_{i}\right\| \leq C h_{e} \quad \text { con } i=5, \ldots, 9, \quad \forall e \in \boldsymbol{\Omega}_{h},
$$

donde $C$ es una constante positiva suficientemente pequeña, entonces la condición inf-sup discreta (2.15) se cumple. Es decir, basta pedir que $h$ sea suficientemente pequeña, para que (2.10) se satisfaga y por ende (2.18). 
Stenberg [26] también muestra que si se cumple la condición inf-sup discreta (2.15) se tienen las siguientes estimaciones de los errores para una familia de elementos isoparamétricos tipo Taylor-Hood:

$$
\begin{aligned}
& \left\|\mathbf{u}-\mathbf{u}_{h}\right\|_{0} \leq C_{1} h^{3}\|\mathbf{u}\|_{3} \quad \mathrm{y} \\
& \left\|q-q_{h}\right\|_{0} \leq C_{2} h^{2}\|q\|_{2}
\end{aligned}
$$

\subsection{Definición de las matrices globales.}

Para obtener el sistema de ecuaciones asociado al problema discreto (2.14), es necesario definir las bases correspondientes a los espacios $\mathbf{V}_{h}, \mathbf{V}_{h}^{0}$ y $Q_{h}^{0}$.

Para ello, se considera que la frontera de $\boldsymbol{\Omega}_{h}$ tiene diferentes condiciones de frontera, es decir, supondremos que $\Gamma_{h}=\Gamma_{\mathrm{D}} \cup \Gamma_{l}$, en donde $\Gamma_{\mathrm{D}}$ contiene los nodos de la frontera que satisfacen las condiciones de Dirichlet (homogéneas y no homoéneas) y $\Gamma_{l}$ involucra a todos los nodos que tienen otras condiciones de frontera, incluyendo las de tipo Neumann.

Definimos a la base usual de $\mathbf{V}_{h}$ por

$$
\mathcal{B}=\left\{\left(\phi_{j}(\mathbf{x}), 0\right),\left(0, \phi_{j}(\mathbf{x})\right)\right\}_{j=1}^{\mathrm{M}},
$$

en donde $\mathrm{M}$ es el número de nodos totales para la velocidad (recordemos que cada elemento $\Omega_{e}$ tiene 9 nodos) y las $\phi_{j}$ son funciones base de Lagrange. La base de $\mathbf{V}_{h}^{0}$ inducida por $\mathbf{V}_{h}$ está definida por

$$
\mathcal{B}^{0}=\left\{\left(\phi_{j}(\mathbf{x}), 0\right),\left(0, \phi_{j}(\mathbf{x})\right)\right\}_{j=1}^{M-L}
$$

en donde $\mathrm{L}$ es el número de nodos que hay sobre $\Gamma_{\mathrm{D}}$. Por último, la base de $Q_{h}$ está dada por

$$
\mathcal{P}=\left\{\gamma_{j}(\mathbf{x})\right\}_{j=1}^{\mathrm{P}}
$$

en donde $\mathrm{P}$ es el número de vértices totales (nodos para la presión) y las $\gamma_{j}$ son las funciones base usuales de Lagrange. El espacio $Q_{h}^{0}$ (veáse (2.11)) tiene la misma base que $Q_{h}$, aunque se fije el valor de la presión en algún vértice para que se satisfaga que dos elementos distintos de $Q_{h}^{0}$ no difieran por una constante. Al valor de la presión que se fija le llamaremos $\widetilde{g}_{\mathrm{p}}$.

Como las funciones bases citadas anteriormente son de Lagrange, cumplen con las propiedades 
siguientes:

$$
\phi_{j}\left(\mathbf{a}_{i}\right)=\delta_{i j}, \quad \text { para } i, j=1, \ldots, \mathrm{M}, \quad y \quad \gamma_{j}\left(\mathbf{a}_{i}\right)=\delta_{i j}, \quad \text { para } i, j=1, \ldots, \mathrm{P},
$$

en donde $\delta_{i j}$ es definida como

$$
\delta_{i j}= \begin{cases}1 & \text { si } i=j \\ 0 & \text { si } i \neq j\end{cases}
$$

Además satisfacen que tanto el soporte de $\phi_{j}$ como el soporte de $\gamma_{j}$ consisten de todos los elementos isoparamétricos $\Omega_{e}$ que tienen como nodo común a $\mathbf{a}_{j}$.

Usando las bases anteriores expresamos a $\mathbf{u}_{h}=\left(\widetilde{u}_{1}, \tilde{u}_{2}\right)$ y a $p_{h}=\tilde{p}$ (por simplicidad se ha suprimido el superíndice $\mathrm{N}$ ) como una combinación lineal de sus respectivas bases, es decir,

$$
\begin{aligned}
\widetilde{u}_{i}(\mathbf{x}) & =\sum_{k=1}^{\mathrm{L}} \widetilde{g}_{i}\left(\mathbf{a}_{k}\right) \phi_{k}(\mathbf{x})+\sum_{j=1}^{\mathrm{M}-\mathrm{L}} \widetilde{u}_{i}\left(\mathbf{a}_{j}\right) \phi_{j}(\mathbf{x}), \quad i=1,2 \quad \mathrm{y} \\
\widetilde{p}(\mathbf{x}) & =\sum_{j=1}^{\mathrm{P}-1} \widetilde{p}\left(\mathbf{a}_{j}\right) \gamma_{j}(\mathbf{x})+\widetilde{g}_{\mathrm{P}} \gamma_{\mathrm{P}}(\mathbf{x})
\end{aligned}
$$

en donde los nodos sobre $\Gamma_{\mathrm{D}}$ cumplen con la condición de Dirichlet: $\mathbf{u}_{h}\left(\mathbf{a}_{j}\right)=\left(\widetilde{g}_{1}\left(\mathbf{a}_{j}\right), \widetilde{g}_{2}\left(\mathbf{a}_{j}\right)\right)$ con $\mathbf{a}_{j} \in \Gamma_{\mathrm{D}}$. Ahora bien, puesto que el problema (2.14) es válido para toda $\mathbf{v}_{h} \in \mathbf{V}_{h}^{0}$, y en particular es cierto para cada una de las funciones base de $\mathbf{V}_{h}^{0}$, se obtiene el siguiente sistema de ecuaciones de Galerkin equivalente a dicho problema (se usa un razonamiento análogo para toda $\left.q_{h} \in Q_{h}^{0}\right)$ :

$$
\begin{array}{rr}
\frac{1}{\Delta t} \int_{\Omega_{h}} \widetilde{u}_{1} \phi_{i} d \Omega+\int_{\Omega_{h}}\left(\widetilde{u}_{1} \frac{\partial \widetilde{u}_{1}}{\partial x}+\widetilde{u}_{2} \frac{\partial \widetilde{u}_{1}}{\partial y}\right) \phi_{i} d \Omega+\frac{1}{R e} \int_{\Omega_{h}}\left(\frac{\partial \widetilde{u}_{1}}{\partial x}\right) \frac{\partial \phi_{i}}{\partial x} d \Omega-\int_{\boldsymbol{\Omega}_{h}} \widetilde{p} \frac{\partial \phi_{i}}{\partial x} d \Omega \\
=\int_{\Omega_{h}} f_{1} \phi_{i} d \Omega+\frac{1}{\Delta t} \int_{\Omega_{h}} \widetilde{u}_{1}^{\mathrm{N}-1} \phi_{i} d \Omega, & i=1, \ldots, \mathrm{M}-\mathrm{L}, \\
\frac{1}{\Delta t} \int_{\boldsymbol{\Omega}_{h}} \widetilde{u}_{2} \phi_{i} d \Omega+\int_{\boldsymbol{\Omega}_{h}}\left(\widetilde{u}_{1} \frac{\partial \widetilde{u}_{2}}{\partial x}+\widetilde{u}_{2} \frac{\partial \widetilde{u}_{2}}{\partial y}\right) \phi_{i} d \Omega+\frac{1}{R e} \int_{\Omega_{h}}\left(\frac{\partial \widetilde{u}_{2}}{\partial y}\right) \frac{\partial \phi_{i}}{\partial y} d \Omega-\int_{\Omega_{h}} \tilde{p} \frac{\partial \phi_{i}}{\partial y} d \Omega \\
=\int_{\Omega_{h}} f_{2} \phi_{i} d \Omega+\frac{1}{\Delta t} \int_{\Omega_{h}} \widetilde{u}_{2}^{\mathrm{N}-1} \phi_{i} d \Omega, & i=1, \ldots, \mathrm{M}-\mathrm{L}, \\
-\int_{\Omega_{h}} \gamma_{i}\left(\frac{\partial \widetilde{u}_{1}}{\partial x}+\frac{\partial \widetilde{u}_{2}}{\partial y}\right) d \Omega=0, & i=1, \ldots, \mathrm{P}-1 .
\end{array}
$$

El sistema no lineal $(2.21)$ tiene $m=2(M-L)+(P-1)$ ecuaciones por $m$ incógnitas. De la misma manera, el sistema lineal asociado al problema discreto (2.13) llamado cuasi-stokes está dado por: 
$\frac{1}{\Delta t} \int_{\Omega_{h}} \widetilde{u}_{1} \phi_{i} d \Omega+\frac{1}{R e} \int_{\Omega_{h}}\left(\frac{\partial \widetilde{u}_{1}}{\partial x}\right) \frac{\partial \phi_{i}}{\partial x} d \Omega-\int_{\Omega_{h}} \widetilde{p} \frac{\partial \phi_{i}}{\partial x} d \Omega=\int_{\Omega_{h}} f_{1} \phi_{i} d \Omega+\frac{1}{\Delta t} \int_{\Omega_{h}} \widetilde{u}_{1}^{N-1} \phi_{i} d \Omega$,

$$
i=1, \ldots, \mathrm{M}-\mathrm{L} \text {, }
$$

$\frac{1}{\Delta t} \int_{\boldsymbol{\Omega}_{h}} \widetilde{u}_{2} \phi_{i} d \Omega+\frac{1}{R e} \int_{\boldsymbol{\Omega}_{h}}\left(\frac{\partial \tilde{u}_{2}}{\partial y}\right) \frac{\partial \phi_{i}}{\partial y} d \Omega-\int_{\boldsymbol{\Omega}_{h}} \tilde{p} \frac{\partial \phi_{i}}{\partial y} d \Omega=\int_{\boldsymbol{\Omega}_{h}} f_{2} \phi_{i} d \Omega+\frac{1}{\Delta t} \int_{\boldsymbol{\Omega}_{h}} \widetilde{u}_{2}^{\mathrm{N}-1} \phi_{i} d \Omega$,

$$
-\int_{\Omega_{h}} \gamma_{i}\left(\frac{\partial \widetilde{u}_{1}}{\partial x}+\frac{\partial \widetilde{u}_{2}}{\partial y}\right) d \Omega=0 \quad i=1, \ldots, \mathrm{P}-1
$$

que en forma matricial se puede escribir como

$$
\left[\begin{array}{ccc}
\mathbf{R} 11 & \mathbf{0} & { }^{t} \mathbf{B} 1 \\
\mathbf{0} & \mathbf{R 2 2} & { }^{t} \mathbf{B 2} \\
\mathbf{B} 1 & \mathbf{B} 2 & \mathbf{0}
\end{array}\right]\left[\begin{array}{c}
\widetilde{\mathbf{u}}_{1}(\mathbf{a}) \\
\widetilde{\mathbf{u}}_{2}(\mathbf{a}) \\
\widetilde{\mathbf{p}}(\mathbf{a})
\end{array}\right]=\left[\begin{array}{l}
\mathbf{b} 1 \\
\mathbf{b} 2 \\
\mathbf{b 3}
\end{array}\right]
$$

en donde

$$
\begin{aligned}
& { }^{t} \widetilde{\mathbf{u}}_{1}(\mathbf{a})=\left(\widetilde{u}_{11}\left(\mathbf{a}_{1}\right), \widetilde{u}_{12}\left(\mathbf{a}_{2}\right), \ldots, \widetilde{u}_{1(\mathbf{M}-\mathrm{L})}\left(\mathbf{a}_{M-L}\right)\right), \\
& { }^{t} \widetilde{\mathbf{u}}_{2}(\mathbf{a})=\left(\widetilde{u}_{21}\left(\mathbf{a}_{1}\right), \widetilde{u}_{22}\left(\mathbf{a}_{2}\right), \ldots, \widetilde{u}_{2(\mathbf{M}-\mathrm{L})}\left(\mathbf{a}_{\mathbf{M}-\mathrm{L}}\right)\right), \\
& { }^{t} \widetilde{\mathbf{p}}(\mathbf{a})=\left(\widetilde{p}_{1}\left(\mathbf{a}_{1}\right), \widetilde{p}_{2}\left(\mathbf{a}_{2}\right), \ldots, \widetilde{p}_{\mathrm{P}-1}\left(\mathbf{a}_{\mathrm{P}-1}\right)\right), \\
& \mathbf{R} 11=\left\{\sum_{e=1}^{\mathbb{N}_{e}} R 11_{i j}^{e}\right\}_{j=1}^{\mathrm{M}-\mathrm{L}}, i=1, \ldots, \mathrm{M}-\mathrm{L} ; \quad \text { con } \quad R 11_{i j}^{e}=\int_{\Omega_{e}}\left[\frac{1}{\Delta t} \phi_{j} \phi_{i}+\frac{1}{R e} \frac{\partial \phi_{j}}{\partial x} \frac{\partial \phi_{i}}{\partial x}\right] d \Omega, \\
& \mathbf{R 2 2}=\left\{\sum_{e=1}^{N_{e}} R 22_{i j}^{e}\right\}_{j=1}^{M-L}, i=1, \ldots, M-L ; \quad \text { con } \quad R 22_{i j}^{e}=\int_{\Omega_{e}}\left[\frac{1}{\Delta t} \phi_{j} \phi_{i}+\frac{1}{R e} \frac{\partial \phi_{j}}{\partial y} \frac{\partial \phi_{i}}{\partial y}\right] d \Omega, \\
& { }^{t} \mathbf{B} 1=\left\{\sum_{e=1}^{\mathrm{N}_{e}}{ }^{t} B 1_{i j}^{e}\right\}_{j=1}^{\mathrm{P}-1}, i=1, \ldots, \mathrm{M}-\mathrm{L} ; \quad \text { con }{ }^{t} B 1_{i j}^{e}=-\int_{\Omega_{e}} \gamma_{j} \frac{\partial \phi_{i}}{\partial x} d \Omega, \\
& \mathbf{B} 1=\left\{\sum_{e=1}^{N_{e}} B 1_{i j}^{e}\right\}_{j=1}^{M-L}, i=1, \ldots, \mathrm{P}-1 ; \quad \text { con } B 1_{i j}^{e}=-\int_{\Omega_{e}} \gamma_{i} \frac{\partial \phi_{j}}{\partial x} d \Omega, \\
& { }^{t} \mathbf{B 2}=\left\{\sum_{e=1}^{\mathrm{N}_{e}}{ }^{t} B 2_{i j}^{e}\right\}_{j=1}^{\mathrm{P}-1}, i=1, \ldots, \mathrm{M}-\mathrm{L} ; \quad \text { con } \quad{ }^{t} B 2_{i j}^{e}=-\int_{\Omega_{e}} \gamma_{j} \frac{\partial \phi_{i}}{\partial y} d \Omega, \\
& \mathbf{B 2}=\left\{\sum_{e=1}^{\mathrm{N}_{e}} B 2_{i j}^{e}\right\}_{j=1}^{\mathrm{M}-\mathrm{L}}, i=1, \ldots, \mathrm{P}-1 ; \quad \text { con } \quad B 2_{i j}^{e}=-\int_{\Omega_{e}} \gamma_{i} \frac{\partial \phi_{j}}{\partial y} d \Omega,
\end{aligned}
$$




$$
\begin{aligned}
& \mathbf{b} \mathbf{1}=\left\{\sum_{e=1}^{\mathrm{N}_{e}} b 1_{i j}^{e}\right\}_{j=1}^{1}, i=1, \ldots, \mathrm{M}-\mathrm{L} ; \quad \text { con } \quad b 1_{i j}^{e}=\int_{\Omega_{e}} f_{1} \phi_{i} d \Omega+\frac{\widetilde{u}_{1 j}^{\mathrm{X}-\mathbf{1}}\left(\mathbf{a}_{j}\right)}{\Delta t} \int_{\Omega e} \phi_{j} \phi_{i} d \Omega \\
& \text { excepto si } \mathbf{a}_{j} \in \Gamma_{\mathrm{D}} \quad \text { entonces } \quad b 1_{i j}^{e}=\int_{\Omega_{e}} f_{1} \phi_{i} d \Omega- \\
& \widetilde{g}_{1}\left(\mathbf{a}_{j}\right) \int_{\Omega_{e}}\left[\frac{1}{\Delta t} \phi_{j} \phi_{i}+\frac{1}{R e} \frac{\partial \phi_{j}}{\partial x} \frac{\partial \phi_{i}}{\partial x}\right] d \Omega-\widetilde{g}_{\mathrm{p}}\left(\mathbf{a}_{\mathrm{P}}\right) \int_{\Omega_{e}} \gamma_{\mathrm{P}} \frac{\partial \phi_{i}}{\partial x} d \Omega+\frac{\widetilde{u}_{1 j}^{N-1}\left(\mathbf{a}_{j}\right)}{\Delta t} \int_{\Omega e} \phi_{j} \phi_{i} d \Omega, \\
& \mathbf{b} 2=\left\{\sum_{e=1}^{\mathrm{N}_{e}} b 2_{i j}^{e}\right\}_{j=1}^{1}, i=1, \ldots, \mathrm{M}-\mathrm{L} ; \quad \text { con } \quad b 2_{i j}^{e}=\int_{\Omega_{e}} f_{2} \phi_{i} d \Omega+\frac{\widetilde{u}_{2 j}^{\mathrm{M}-1}\left(\mathbf{a}_{j}\right)}{\Delta t} \int_{\Omega e} \phi_{j} \phi_{i} d \Omega \\
& \text { excepto si } \mathrm{a}_{j} \in \Gamma_{\mathrm{D}} \quad \text { entonces } \quad b 2_{i j}^{e}=\int_{\Omega_{e}} f_{2} \phi_{i} d \Omega- \\
& \widetilde{g}_{2}\left(\mathbf{a}_{j}\right) \int_{\Omega_{e}}\left[\frac{1}{\Delta t} \phi_{j} \phi_{i}+\frac{1}{R e} \frac{\partial \phi_{j}}{\partial y} \frac{\partial \phi_{i}}{\partial y}\right] d \Omega-\widetilde{g}_{\mathrm{P}}\left(\mathbf{a}_{\mathrm{p}}\right) \int_{\Omega_{e}} \gamma_{\mathrm{p}} \frac{\partial \phi_{i}}{\partial y} d \Omega+\frac{\widetilde{u}_{2 j}^{\mathrm{N}-1}\left(\mathbf{a}_{j}\right)}{\Delta t} \int_{\Omega e} \phi_{j} \phi_{i} d \Omega, \\
& \mathbf{b} \mathbf{3}=\left\{\sum_{e=1}^{N_{e}} b 3_{i j}^{e}\right\}_{j=1}^{1}, i=1, \ldots, \mathrm{P}-1 ; \quad \text { con } \quad b 3_{i j}^{e}=0, \text { excepto si } \mathbf{a}_{j} \in \Gamma_{\mathrm{D}} \text { entonces } \\
& b 3_{i j}^{e}=\widetilde{g}_{1}\left(\mathbf{a}_{j}\right) \int_{\Omega_{e}} \gamma_{i} \frac{\partial \phi_{j}}{\partial x} d \Omega+\widetilde{g}_{2}\left(\mathbf{a}_{j}\right) \int_{\Omega_{e}} \gamma_{i} \frac{\partial \phi_{j}}{\partial y} d \Omega \text {. }
\end{aligned}
$$

La matriz del problema (2.23) de tamaño $\mathrm{m} \times \mathrm{m}$ se denotará por $\mathbf{A}$, y al vector constante se denotará por b. Observemos que la matriz $\mathbf{A}$ es simétrica, ya que $\mathbf{R 1 1}={ }^{t} \mathbf{R} 11,{ }^{t} \mathbf{R 2 2}=\mathbf{R 2 2}$, ${ }^{t}\left({ }^{t} \mathbf{B 1}\right)=\mathbf{B 1} \quad \mathrm{y}^{t}\left({ }^{t} \mathbf{B 2}\right)=\mathbf{B 2}$. Más aún, la matriz $\mathbf{A}$ es rala (sparse), puesto que $A_{i j}=0$ a menos que $\mathbf{a}_{i}$ y $\mathbf{a}_{j}$ sean nodos del mismo elemento (recordemos que el soporte de las funciones base $\phi_{j}$ y $\gamma_{j}$ es pequeño).

\subsection{Definición de las matrices locales.}

En el apartado anterior se hizo ver que el cálculo de las componentes $A_{i j}$ de la matriz $\mathbf{A}$ se efectúa a través de cada una de las contribuciones de los elementos $\Omega_{e}$, es decir,

$$
A_{i j}=\sum_{e=1}^{\mathrm{N}_{e}} A_{i j}^{e} .
$$

Por otro lado, definiremos a la matriz $\mathbf{A}^{e}=\left\{A_{i j}^{e}\right\}_{j=1}^{m}$ con $i=1, \ldots, \mathrm{m}$, como la matriz elemental del elemento $\Omega_{e}$. Luego la matriz A se puede escribir como la suma de las matrices elementales $\mathbf{A}^{e}$, o sea, 


$$
\mathbf{A}=\sum_{e=1}^{\mathbf{N}_{e}} \mathbf{A}^{e}
$$

Cada matriz elemental $\mathrm{A}^{e}$ es rala puesto que $A_{i j}^{e}=0$ a menos que $\mathbf{a}_{i}$ y $\mathbf{a}_{j}$ pertenezcan al elemento $\Omega_{e}$. Observese que $A_{i j}^{e}$ puede ser $R 11_{i j}^{e}, R 22_{i j}^{e},{ }^{t} B 1_{i j}^{e}, B 1_{i j}^{e},{ }^{t} B 2_{i j}^{e}$ o $B 2_{i j}^{e}$. Definimos a la matriz local $\overline{\mathbf{A}}^{e}$ como aquella matriz de tamaño $22 \times 22$ que contiene la información asociada a los nodos $\mathbf{a}_{i}$ y $\mathbf{a}_{j}$ que están en el elemento $\Omega_{e}$, es decir,

$$
\overline{\mathbf{A}}^{e}=\left[\begin{array}{ccc}
\overline{\mathbf{R} 11}^{e} & 0 & \bar{t}^{{ }^{\mathrm{B1}}}{ }^{e} \\
0 & \overline{\mathbf{R 2 2}}^{e} & \bar{t}^{\overline{\mathbf{B 2}}} \\
\overline{\mathbf{B 1}}^{e} & \overline{\mathbf{B 2}}^{e} & 0
\end{array}\right]
$$

en donde $\overline{\mathbf{R 1 1}}^{e}$, y $\overline{\mathbf{R 2 2}}^{e}$ son submatrices de tamaño $9 \times 9$ tal que

$$
\begin{aligned}
& \overline{R 11}_{i j}^{e}=\int_{\Omega_{e}}\left[\frac{1}{\Delta t} \psi_{j}^{e} \psi_{i}^{e}+\frac{1}{R e} \frac{\partial \psi_{j}^{e}}{\partial x} \frac{\partial \psi_{i}^{e}}{\partial x}\right] d \Omega \\
& \overline{R 22}_{i j}^{e}=\int_{\Omega_{e}}\left[\frac{1}{\Delta t} \psi_{j}^{e} \psi_{i}^{e}+\frac{1}{R e} \frac{\partial \psi_{j}^{e}}{\partial y} \frac{\partial \psi_{i}^{e}}{\partial y}\right] d \Omega
\end{aligned}
$$

mientras que $\bar{t}^{e}{ }^{e}$ y $\overline{ }^{t} \overline{\mathbf{B 2}}^{e}$ son submatrices de tamaño $9 \times 4$ tales que

$$
\begin{aligned}
& \overline{ }_{{ }^{t} B 1_{i j}^{e}}^{e}=-\int_{\Omega_{e}} \varphi_{j}^{e} \frac{\partial \psi_{i}^{e}}{\partial x} d \Omega, \\
& \overline{ }_{{ }^{t} B 2_{i j}^{e}}^{e}=-\int_{\Omega_{e}} \varphi_{j}^{e} \frac{\partial \psi_{i}^{e}}{\partial y} d \Omega .
\end{aligned}
$$

Por último, $\overline{\mathbf{B 1}}^{e}$ y $\overline{\mathbf{B 2}}^{e}$ son submatrices de tamaño $4 \times 9$ tal que

$$
\begin{aligned}
& \overline{B 1}_{i j}^{e}=-\int_{\Omega_{e}} \varphi_{i}^{e} \frac{\partial \psi_{j}^{e}}{\partial x} d \Omega \\
& \overline{B 2}_{i j}^{e}=-\int_{\Omega_{e}} \varphi_{i}^{e} \frac{\partial \psi_{j}^{e}}{\partial y} d \Omega .
\end{aligned}
$$

Usando un procedimiento análogo, se define el vector local $\overline{\mathbf{b}}^{e}$ como

$$
\overline{\mathbf{b}}^{e}=\left[\begin{array}{c}
\overline{\mathbf{b}}_{1}^{e} \\
\overline{\mathbf{b}}_{2}^{e} \\
\overline{\mathbf{b}}_{3}^{e}
\end{array}\right]
$$


en donde los subvectores $\overline{\mathbf{b}}_{1}^{e}$ y $\overline{\mathbf{b}}_{2}^{e}$ de tamaño $9 \times 1$, y el subvector $\overline{\mathbf{b}}_{3}^{e}$ de tamaño $4 \times 1$ son tales que

$$
\begin{aligned}
& \overline{b 1}_{i j}^{e}=\int_{\Omega_{e}} f_{1} \psi_{i}^{e} d \Omega-\widetilde{g}_{1}\left(\mathbf{a}_{j}\right) \int_{\Omega_{e}}\left[\frac{1}{\Delta t} \psi_{j}^{e} \psi_{i}^{e}+\frac{1}{R e} \frac{\partial \psi_{j}^{e}}{\partial x} \frac{\partial \psi_{i}^{e}}{\partial x}\right] d \Omega- \\
& \tilde{g}_{\mathrm{P}}\left(\mathrm{a}_{\mathrm{P}}\right) \int_{\Omega_{e}} \varphi_{\mathrm{P}}^{e} \frac{\partial \psi_{i}^{e}}{\partial x} d \Omega+\frac{\tilde{u}_{1 j}^{N-1}\left(\mathbf{a}_{j}\right)}{\Delta t} \int_{\Omega e} \psi_{j}^{e} \psi_{i}^{e} d \Omega, \\
& \overline{b 2}_{i j}^{e}=\int_{\Omega_{e}} f_{2} \psi_{i}^{e} d \Omega-\widetilde{g}_{2}\left(\mathbf{a}_{j}\right) \int_{\Omega_{e}}\left[\frac{1}{\Delta t} \psi_{j}^{e} \psi_{i}^{e}+\frac{1}{R e} \frac{\partial \psi_{j}^{e}}{\partial y} \frac{\partial \psi_{i}^{e}}{\partial y}\right] d \Omega- \\
& \tilde{g}_{\mathrm{P}}\left(\mathrm{a}_{\mathrm{P}}\right) \int_{\Omega_{e}} \varphi_{\mathrm{P}}^{e} \frac{\partial \psi_{i}^{e}}{\partial y} d \Omega+\frac{\tilde{u}_{2 j}^{\mathrm{N}-1}\left(\mathbf{a}_{j}\right)}{\Delta t} \int_{\Omega e} \psi_{j}^{e} \psi_{i}^{e} d \Omega, \\
& {\overline{b \overline{3}_{i j}}}_{i j}=\widetilde{g}_{1}\left(\mathbf{a}_{j}\right) \int_{\Omega_{e}} \varphi_{i}^{e} \frac{\partial \psi_{j}^{e}}{\partial x} d \Omega+\widetilde{g}_{2}\left(\mathbf{a}_{j}\right) \int_{\Omega_{e}} \varphi_{i}^{e} \frac{\partial \psi_{j}^{e}}{\partial y} d \Omega \text {. }
\end{aligned}
$$

Por lo tanto, para calcular las componentes de la matriz global $\mathbf{A}$ y el vector global $\mathbf{b}$, basta con evaluar las matrices locales $\overline{\mathbf{A}}^{e}$ y $\overline{\mathbf{b}}^{e}$ para cada $e=1, \ldots, N_{e}$. Por ello, es suficiente trabajar con las restricciones de las funciones base $\phi_{j}$ y $\gamma_{j}$ sobre cada elemento isoparamétrico $\Omega_{e}$, las cuales las denotamos por

$$
\begin{gathered}
\left.\phi_{j}\right|_{\Omega_{e}}=\psi_{i}^{e} \\
\left.\gamma_{j}\right|_{\Omega_{e}}=\varphi_{i}^{e}
\end{gathered}
$$

en donde las funciones base locales $\psi_{i}^{e} \mathrm{y} \varphi_{i}^{e}$ se definen en el apartado siguiente.

\subsection{Definición de las funciones base locales y descripción de sus derivadas.}

En el apartado 2.2 .1 se vio que cada $\Omega_{e} \in \Omega_{h}$ es un elemento isoparamétrico, obtenido a través de la transformación $T_{e}$, es decir que $T_{e}\left(\Omega_{\hat{e}}\right)=\Omega_{e}$. Además en (2.2) se exhibió la forma de las funciones $\hat{\psi}_{i}, i=1, \ldots, 9$, que generan al espacio $\hat{\mathrm{Q}}_{2}\left(\Omega_{\hat{e}}\right)$. Estas funciones se utilizan para definir las funciones base $\psi_{i}^{e}$, con $i=1, \ldots, 9$, que generan al espacio $Q_{2}\left(\Omega_{e}\right)$, ya que por la condición (2.6), $\psi_{i}^{e}=\hat{\psi}_{i} \circ \mathbf{T}_{e}^{-1}$, es decir,

$$
\psi_{i}^{e}(x, y)=\hat{\psi}_{i}(\xi(x, y), \eta(x, y)) \quad \text { para } i=1, \ldots, 9
$$

Las funciones $\psi_{i}^{e}$, con $i=1, \ldots, 9$, son llamadas funciones base locales del elemento $\Omega_{e}$ para la velocidad.

De manera similar las funciones base locales del elemento $\Omega_{e}$, para la presión, que generan 
el espacio $Q_{1}\left(\Omega_{e}\right)$ están dadas por

$$
\varphi_{i}^{e}(x, y)=\widehat{\varphi}_{i}(\xi(x, y), \eta(x, y)) \quad \text { para } i=1, \ldots, 4
$$

en donde las funciones $\widehat{\varphi}_{i}$ están definidas en (2.1).

Para exhibir cómo se calculan las derivadas $\frac{\partial \psi_{i}^{e}}{\partial x}$ y $\frac{\partial \psi_{i}^{e}}{\partial y}$ se necesitan los resultados o relaciones que a continuación se desarrollan:

De (2.8) con $\mathrm{N}_{T}=9$ se observa que $x=x(\xi, \eta), y=y(\xi, \eta)$ son continuamente diferenciables con respecto a $\xi$ y $\eta$. Luego, las diferenciales $d x$ y $d y$ son dadas por

$$
d x=\frac{\partial x}{\partial \xi} d \xi+\frac{\partial x}{\partial \eta} d \eta \quad \text { y } \quad d y=\frac{\partial y}{\partial \xi} d \xi+\frac{\partial y}{\partial \eta} d \eta
$$

o en forma matricial

$$
\left[\begin{array}{l}
d x \\
d y
\end{array}\right]=\left[\begin{array}{ll}
\frac{\partial x}{\partial \xi} & \frac{\partial x}{\partial \eta} \\
\frac{\partial y}{\partial \xi} & \frac{\partial y}{\partial \eta}
\end{array}\right]\left[\begin{array}{l}
d \xi \\
d \eta
\end{array}\right]
$$

A la matriz en la relación anterior se le llama la matriz Jacobiana de la transformación (2.8) y es denotada por $\mathbf{J}(\xi, \eta)$ y a su determinante $|\mathbf{J}(\xi, \eta)|$ se le conoce como el Jacobiano de la transformación $T_{e}$, definido por

$$
|\mathbf{J}(\xi, \eta)|=\operatorname{det} \mathbf{J}(\xi, \eta)=\frac{\partial x}{\partial \xi} \frac{\partial y}{\partial \eta}-\frac{\partial x}{\partial \eta} \frac{\partial y}{\partial \xi}
$$

Se sabe que si $|\mathbf{J}(\xi, \eta)| \neq 0$ la transformación $T_{e}^{-1}$ existe, y como no se quiere que alguna porción de $\Omega_{\hat{e}}$ "cambie de cara" al mapearse en el plano $x-y$, se pide que $|\mathbf{J}(\xi, \eta)|>0$ en todo $\Omega_{\hat{e} \text {. }}$

Luego si $|\mathbf{J}(\xi, \eta)|>0$, se puede escribir

$$
\left[\begin{array}{l}
d \xi \\
d \eta
\end{array}\right]=\mathbf{J}(\xi, \eta)^{-1}\left[\begin{array}{l}
d x \\
d y
\end{array}\right]=\frac{1}{|\mathbf{J}(\xi, \eta)|}\left[\begin{array}{cc}
\frac{\partial y}{\partial \eta} & -\frac{\partial x}{\partial \eta} \\
-\frac{\partial y}{\partial \xi} & \frac{\partial x}{\partial \xi}
\end{array}\right]\left[\begin{array}{l}
d x \\
d y
\end{array}\right]
$$

Por otro lado, dado que

$$
(\xi, \eta)=T_{e}^{-1}(x, y)
$$

es una transformación que mapea a $\Omega_{e}$ en el elemento de referencia $\Omega_{\hat{e}}$, se obtiene en forma 
análoga a (2.39) que

$$
\left[\begin{array}{l}
d \xi \\
d \eta
\end{array}\right]=\left[\begin{array}{ll}
\frac{\partial \xi}{\partial x} & \frac{\partial \xi}{\partial y} \\
\frac{\partial \eta}{\partial x} & \frac{\partial \eta}{\partial y}
\end{array}\right]\left[\begin{array}{l}
d x \\
d y
\end{array}\right]
$$

Igualando los términos de las ecuaciones (2.41) y (2.42) se llega a que

$$
\begin{array}{ll}
\frac{\partial \xi}{\partial x}=\frac{1}{|\mathbf{J}(\xi, \eta)| \frac{\partial y}{\partial \eta},} & \frac{\partial \xi}{\partial y}=-\frac{1}{|\mathbf{J}(\xi, \eta)|} \frac{\partial x}{\partial \eta}, \\
\frac{\partial \eta}{\partial x}=-\frac{1}{|\mathbf{J}(\xi, \eta)| \frac{\partial y}{\partial \xi},} & \frac{\partial \eta}{\partial y}=\frac{1}{|\mathbf{J}(\xi, \eta)|} \frac{\partial x}{\partial \xi} .
\end{array}
$$

Utilizando la definición de la trasformación (2.8) se tienen las ecuaciones

$$
\begin{array}{ll}
\frac{\partial x}{\partial \xi}=\sum_{k=1}^{9} x_{k} \frac{\partial \hat{\psi}_{k}}{\partial \xi}, & \frac{\partial x}{\partial \eta}=\sum_{k=1}^{9} x_{k} \frac{\partial \hat{\psi}_{k}}{\partial \eta}, \\
\frac{\partial y}{\partial \xi}=\sum_{k=1}^{9} y_{k} \frac{\partial \hat{\psi}_{k}}{\partial \xi}, & \frac{\partial y}{\partial \eta}=\sum_{k=1}^{9} y_{k} \frac{\partial \hat{\psi}_{k}}{\partial \eta} .
\end{array}
$$

Las derivadas de $\psi_{i}^{e}$ son obtenidas aplicando la regla de la cadena a (2.37):

$$
\frac{\partial \psi_{i}^{e}}{\partial x}=\frac{\partial \hat{\psi}_{i}}{\partial \xi} \frac{\partial \xi}{\partial x}+\frac{\partial \hat{\psi}_{i}}{\partial \eta} \frac{\partial \eta}{\partial x} ; \quad \frac{\partial \psi_{i}^{e}}{\partial y}=\frac{\partial \hat{\psi}_{i}}{\partial \xi} \frac{\partial \xi}{\partial y}+\frac{\partial \hat{\psi}_{i}}{\partial \eta} \frac{\partial \eta}{\partial y}
$$

Sustituyendo las ecuaciones (2.44) en (2.43), y estos resultados a su vez, en las igualdades (2.45) se deduce que

$$
\begin{gathered}
\frac{\partial \psi_{i}^{e}}{\partial x}=\frac{1}{|\mathbf{J}(\xi, \eta)|}\left\{\frac{\partial \hat{\psi}_{i}}{\partial \xi} \sum_{k=1}^{9} y_{k} \frac{\partial \hat{\psi}_{k}}{\partial \eta}-\frac{\partial \hat{\psi}_{i}}{\partial \eta} \sum_{k=1}^{9} y_{k} \frac{\partial \hat{\psi}_{k}}{\partial \xi}\right\}, \\
\frac{\partial \psi_{i}^{e}}{\partial y}=\frac{1}{|\mathbf{J}(\xi, \eta)|}\left\{-\frac{\partial \hat{\psi}_{i}}{\partial \xi} \sum_{k=1}^{9} x_{k} \frac{\partial \hat{\psi}_{k}}{\partial \eta}+\frac{\partial \hat{\psi}_{i}}{\partial \eta} \sum_{k=1}^{9} x_{k} \frac{\partial \hat{\dot{\psi}}_{k}}{\partial \xi}\right\},
\end{gathered}
$$

en donde

$$
|\mathbf{J}(\xi, \eta)|=\left\{\sum_{k=1}^{9} x_{k} \frac{\partial \hat{\psi}_{k}}{\partial \xi}\right\}\left\{\sum_{k=1}^{9} y_{k} \frac{\partial \hat{\psi}_{k}}{\partial \eta}\right\}-\left\{\sum_{k=1}^{9} x_{k} \frac{\partial \hat{\psi}_{k}}{\partial \eta}\right\}\left\{\sum_{k=1}^{9} y_{k} \frac{\partial \hat{\psi}_{k}}{\partial \xi}\right\} .
$$

Esta última relación se encuentra de sustituir (2.44) en (2.40).

Por lo tanto, para evaluar las funciones de forma $\psi_{i}^{e}$ y $\varphi_{i}^{e}$ se utilizan las relaciones (2.37) y (2.38) respectivamente. Además, para calcular las derivadas parciales $\frac{\partial \psi_{i}^{e}}{\partial x}$ y $\frac{\partial \psi_{i}^{e}}{\partial y}$ se ocupan las relaciones $(2.46)$ y $(2.47)$. 
En este capítulo se ha obtenido la discretización de los problemas de Stokes y de NavierStokes, se expreso cómo se construían los elementos y transformaciones isoparamétricas y cómo se formaban las matrices globales y locales. En el capítulo siguiente se verá cómo se calculan numéricamente las componentes de estas matrices. 


\section{Capítulo 3}

\section{Métodos de solución y descripción del programa NAVSTOKE}

En este capítulo se especifica cual es el método de integración numérica que se utiliza para calcular las componentes de las matrices locales (2.31), se describe el algoritmo que se usa para almacenar la matriz global y se presentan los algoritmos que resuelven los sistemas de ecuaciones lineales y no lineales. Además se mencionan las condiciones para que el esquema de discretización sea estable, y para que el método iterativo para resolver los sistemas no lineales resultantes sea convergente. Por último se da una breve descripción del programa NAVSTOKE que usando el método de elemento finito, resuelve numéricamente las ecuaciones de NavierStokes o de Stokes estacionarias y no estacionarias con condiciones de frontera tipo Dirichlet y Neumann sobre regiones curvas.

\subsection{Método de integración numérica.}

Para evaluar las matrices locales (2.31) y los vectores locales (2.35), se requiere calcular las integrales dadas en las relaciones (2.32)-(2.34) y (2.36). Para ello, observemos que si $r$ es una función de $x, y$ definida sobre el elemento $\Omega_{e}$, se puede convertir a $r$ en una función $\widehat{r}$ de $\xi$ y $\eta$ 
definida sobre $\Omega_{\hat{e}}$ por medio de

$$
r(x, y)=r(x(\xi, \eta), y(\xi, \eta))=\widehat{r}(\xi, \eta)
$$

en donde $x(\xi, \eta)$ y $y(\xi, \eta)$ están dadas en (2.8). Luego, es claro que

$$
\int_{\Omega_{e}} r(x, y) d x d y=\int_{\Omega_{\hat{e}}} \widehat{r}(\xi, \eta)|\mathbf{J}(\xi, \eta)| d \xi d \eta
$$

Si se sustituye (2.8) con (3.1), se obtiene que $\widehat{r}$ está determinada por

$$
\widehat{r}(\xi, \eta)=r\left(\sum_{j=1}^{9} x_{j} \hat{\psi}_{j}(\xi, \eta), \sum_{j=1}^{9} y_{j} \hat{\psi}_{j}(\xi, \eta)\right)
$$

Luego, las integrales sobre $\Omega_{e}(2.32)-(2.34)$ y (2.36) se pueden evaluar sobre $\Omega_{\hat{e}}$ usando únicamente las expresiones $(2.37),(2.38),(2.46)$ y $(2.47)$, ya que se cumple (3.3)

La regla de cuadratura Gauss-Legendre para 3 puntos en una dimensión es exacta en el espacio de funciones $\mathrm{P}_{5}([-1,1])$, en donde $\mathrm{P}_{5}$ se definió en el apartado 2.2 .1 (veáse Ciarlet [5]) . Además, siempre que $\mathrm{P}_{2}\left(\Omega_{\hat{e}}\right) \subset \hat{\mathrm{Q}}_{2}\left(\Omega_{\hat{e}}\right) \subset \mathrm{Q}_{2}\left(\Omega_{\hat{e}}\right)$ la fórmula de cuadratura de Gauss de 9 puntos para el cuadrilátero $\Omega_{\hat{e}}$ es exacta en el espacio $\hat{Q}_{5}\left([-1,1]^{2}\right)$.

Debido a que $\psi_{i}^{e} \in Q_{2}\left(\Omega_{e}\right)$ y $\varphi_{i}^{e} \in Q_{1}\left(\Omega_{e}\right)$, y puesto que las componentes de $\overline{\mathbf{A}}^{e}$ y $\overline{\mathbf{b}}^{e}$ dependen básicamente de ellas y de sus derivadas, se usa una fórmula de cuadratura de Gauss de 9 puntos para realizar la integración numérica, la cual se aplica a la ecuación (3.3) de la siguiente manera:

$$
\int_{\Omega_{e}} r(x, y) d x d y=\int_{\Omega_{\hat{e}}} \widehat{r}(\xi, \eta)|\mathbf{J}(\xi, \eta)| d \xi d \eta=\sum_{l=1}^{9} \widehat{r}\left(\xi_{l}, \eta_{l}\right)\left|\mathbf{J}\left(\xi_{l}, \eta_{l}\right)\right| w_{l} d \xi d \eta+\hat{E}
$$

en donde $\hat{E}$ es error de cuadratura, los $\left(\xi_{l}, \eta_{l}\right) \in \Omega_{\hat{e}}$ son los puntos de integración y $w_{l}$ son los pesos de integración cuyos valores son: 


$\begin{array}{cccc}l & \xi_{l} & \eta_{l} & w_{l} \\ 1 & -\sqrt{3 / 5} & -\sqrt{3 / 5} & 25 / 81 \\ 2 & 0 & -\sqrt{3 / 5} & 40 / 81 \\ 3 & \sqrt{3 / 5} & -\sqrt{3 / 5} & 25 / 81 \\ 4 & -\sqrt{3 / 5} & 0 & 40 / 81 \\ 5 & 0 & 0 & 64 / 81 \\ 6 & \sqrt{3 / 5} & 0 & 40 / 81 \\ 7 & -\sqrt{3 / 5} & \sqrt{3 / 5} & 25 / 81 \\ 8 & 0 & \sqrt{3 / 5} & 40 / 81 \\ 9 & \sqrt{3 / 5} & \sqrt{3 / 5} & 25 / 81\end{array}$

\subsection{Método de almacenamiento de la matriz global A.}

Para almacenar la matriz global A (veáse (2.23)), se usa el método de Perfil (o Skyline), ya que en el proceso de eliminación Gaussiana las entradas que están fuera del perfil de la matriz siguen siendo cero, por lo que solamente se usa el perfil de la matriz para la factorización. Además, hay algunos casos en donde resultan ser más baratos estos métodos que los métodos de banda (veáse Cuvelier [7]). A continuación se definirá lo que es el perfil de una matriz y se dirá en que consiste el método de perfil (o Skyline).

El perfil de una matriz se define en la forma siguiente: consideramos el renglón i-ésimo, entonces todos los elementos del renglón i-ésimo de la matriz triangular inferior y todos los elementos que estén en la columna i-ésima sobre la matriz triangular superior, situados entre dos elementos exteriores distintos de cero pertenecen al perfil de la matriz incluyendo a estos últimos. Por ejemplo, si se tiene la matriz dada en (3.7). El perfil de la matriz $\mathbf{A}$ almacenado en un arreglo unidimensional es:

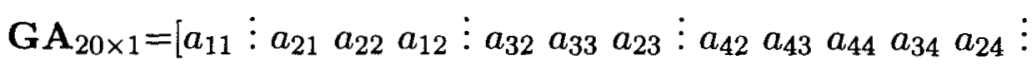

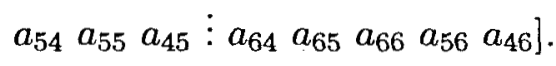




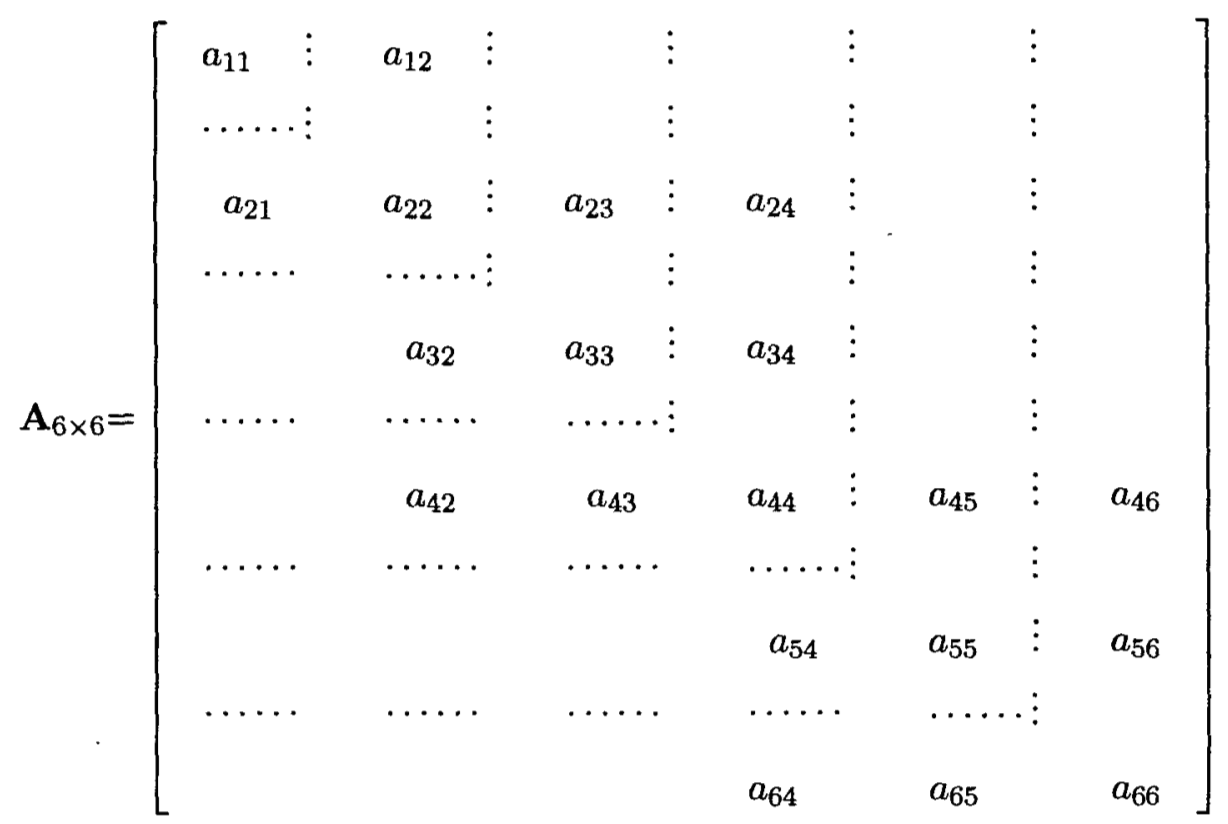

En caso de que la matriz A sea simétrica, sólo se almacena la parte inferior del perfil de la matriz, es decir,

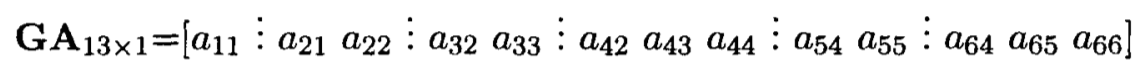

El método del perfil consiste en construir dos vectores $\mathrm{GA}_{\mathrm{G} \times 1}$ y $\mathbf{M U A 4 _ { \mathrm { m } + 1 \times 1 }}$ (en donde $\mathrm{G}$ es el número de elementos que pertenecen al perfil), en tal forma que GA almacene los elementos del perfil en la forma descrita en (3.6) o (3.8), según sea el caso. Y el vector apuntador MUA4 nos dice en que posición del vector GA se encuentra cada elemento de la diagonal de $\mathbf{A}$, es decir, si MUA4 $(5+1)=13$ indica que el elemento de la diagonal del quinto renglón de la matriz global se encuentra en la decimatercera posición del vector GA. Observemos que en algunos compiladores de Fortrán los arreglos comienzan en "cero", por lo cual tendríamos que MUA4 (5) $=13$.

\subsection{Métodos de resolución.}

Este apartado se divide básicamente en dos partes. En la primera se describen los métodos que se utilizan para resolver el sistema de ecuaciones lineales (2.22), obtenido del problema discreto de cuasi-Stokes (2.13). En la segunda parte se presenta cómo se trata la parte no lineal del 
problema discreto de Navier-Stokes (2.14) a partir de la solución del problema correspondiente de cuasi-Stokes.

\subsubsection{Sistemas de ecuaciones lineales.}

Observemos que el sistema de ecuaciones lineales descrito en (2.23)-(2.30) tiene asociada la matriz A que es simétrica y rala, pero que no tiene estructura, lo que hace difícil encontrar un método numérico que resuelva eficientemente al sistema, para ello se necesitaría otra tesis de maestría. Por lo tanto se utilizó en este trabajo el método de Crout adaptado para el método del perfil, porque explota las características de la matriz A de ser simétrica y rala.

El método de Crout se divide básicamente en dos etapas. La primera etapa factoriza la matriz $\mathbf{A}$ como $\mathbf{L D}^{t} \mathbf{L}$, donde $\mathbf{L}$ es una matriz triangular inferior y $\mathbf{D}$ es una matriz diagonal, y la segunda etapa resuelve los sistemas

$$
\begin{aligned}
& \mathbf{L y}=\mathbf{b} \\
& \mathbf{D z}=\mathbf{y} \\
& \mathbf{L u}=\mathbf{z}
\end{aligned}
$$

Además como la matriz A es almacenada en el vector GA, ambas etapas toman en cuenta dicha condición. El algoritmo de este método se encuentra en Perronnet [22], el cual se modifica para que la factorización de Crout $\mathbf{L D}^{t} \mathbf{L}$ sea almacenada en el vector $\mathbf{G A}$.

\subsubsection{Sistemas de ecuaciones no lineales.}

El método utilizado para resolver el sistema de ecuaciones no lineales descrito en (2.21), que es equivalente a la discretación del problema de Navier-Stokes "estacionario" definido en (2.14), es el método iterativo de Punto Fijo. Se escogió este método porque se conoce que es convergente como lo muestran Karakashian [17] y Juárez-Valencia [16].

El método iterativo de punto fijo consiste en los pasos siguientes:

(i) En la inicialización $K=0$, se resuelve el problema cuasi-Stokes en la forma descrita en el 
apartado 3.3.1. La solución de este problema es el vector $\mathbf{u}_{h}^{(0)}=\left[\begin{array}{c}\widetilde{\mathbf{u}}_{1}(\mathbf{a})^{(0)} \\ \widetilde{\mathbf{u}}_{2}(\mathbf{a})^{(0)} \\ \widetilde{\mathbf{p}}(\mathbf{a})^{(0)}\end{array}\right]$, en donde $\widetilde{\mathbf{u}}_{1}(\mathbf{a}), \widetilde{\mathbf{u}}_{2}(\mathbf{a})$ y $\widetilde{\mathbf{p}}(\mathbf{a})$ están definidas en $(2.24)$.

(ii) Para $K=1,2,3, \ldots \ldots$, se resuelve

$$
\begin{gathered}
a\left(\mathbf{u}_{h}^{(\mathrm{K})}, \mathbf{v}_{h}\right)+b\left(p_{h}^{(\mathrm{K})}, \mathbf{v}_{h}\right)=L_{\boldsymbol{f}}\left(\mathbf{v}_{h}\right)-a_{1}\left(\mathbf{u}_{h}^{(\mathrm{K}-1)} ; \mathbf{u}_{h}^{(\mathrm{K}-1)}, \mathbf{v}_{h}\right) \quad \forall \mathbf{v}_{h} \in \mathbf{V}_{h}^{0}, \\
b\left(q_{h}, \mathbf{u}_{h}^{(\mathrm{K})}\right)=0 \quad \forall q_{h} \in Q_{h}^{0} .
\end{gathered}
$$

hasta que $\frac{\left\|\mathbf{u}_{h}^{(K)}-\mathbf{u}_{h}^{(\mathrm{K}-1)}\right\|_{0}}{\left\|\mathbf{u}_{h}^{(\kappa)}\right\|_{0}} \leq 10^{-8} \quad$ o $\quad \mathrm{K}>$ MAXIT1.

Notemos que los operadores $a(\cdot, \cdot)$ y $L_{f}(\cdot)$ están definidos en $(1.20)$ y que las relaciones (3.10) son equivalentes al sistema lineal:

$$
\left[\begin{array}{ccc}
\mathbf{R} 11 & \mathbf{0} & { }^{t} \mathbf{B 1} \\
\mathbf{0} & \mathbf{R 2 2} & { }^{t} \mathbf{B 2} \\
\mathbf{B 1} & \mathbf{B 2} & \mathbf{0}
\end{array}\right]\left[\begin{array}{c}
\widetilde{\mathbf{u}}_{1}(\mathbf{a})^{(\mathrm{K})} \\
\widetilde{\mathbf{u}}_{2}(\mathbf{a})^{(\mathrm{K})} \\
\widetilde{\mathbf{p}}(\mathbf{a})^{(\mathrm{K})}
\end{array}\right]=\left[\begin{array}{c}
\mathbf{b} 1+\mathbf{k} \mathbf{1}^{(\mathrm{K}-1)} \\
\mathbf{b} 2+\mathbf{k 2}^{(\mathrm{K}-1)} \\
\mathbf{b 3}
\end{array}\right]
$$

en donde las matrices $\mathbf{R 1 1}, \mathbf{R 2 2},{ }^{t} \mathbf{B 1}, \mathbf{B 1},{ }^{t} \mathbf{B 2}, \mathbf{B 2}, \mathbf{b 1}$, b2 y b3 están definidas en (2.25)(2.30), respectivamente. Además

$$
\begin{array}{r}
\mathbf{k} \mathbf{1}^{(\mathrm{K}-1)}=\left\{\sum_{e=1}^{\mathrm{N}_{e}} k 1_{i j}^{e,(\mathrm{~K}-1)}\right\}_{j=1}^{1}, \quad i=1, \ldots, \mathrm{M}-\mathrm{L} ; \quad \text { con } \\
k \mathbf{1}_{i j}^{e,(\mathrm{~K}-1)}=-\int_{\Omega_{e}}\left[\left(\sum_{k=1}^{\mathrm{M}} \widetilde{u}_{1 k}^{(\mathrm{K}-1)}\left(\mathbf{a}_{k}\right) \phi_{k}\right)\left(\sum_{l=1}^{\mathrm{M}} \widetilde{u}_{1 l}^{(\mathrm{K}-1)}\left(\mathbf{a}_{l}\right) \frac{\partial \phi_{l}}{\partial x}\right)+\right. \\
\left.\left(\sum_{k=1}^{\mathrm{M}} \widetilde{u}_{2 k}^{(\mathrm{K}-1)}\left(\mathbf{a}_{k}\right) \phi_{k}\right)\left(\sum_{l=1}^{\mathrm{M}} \widetilde{u}_{1 l}^{(\mathrm{K}-1)}\left(\mathbf{a}_{l}\right) \frac{\partial \phi_{l}}{\partial y}\right)\right] \phi_{i} d \Omega, \\
\mathbf{k} \mathbf{2}^{(\mathrm{K}-1)}=\left\{\sum_{e=1}^{\mathrm{N}_{e}} k 2_{i j}^{e_{i}(\mathrm{~K}-1)}\right\}_{j=1}^{1}, \quad i=1, \ldots, \mathrm{M}-\mathrm{L} ; \quad \text { con } \\
k 2_{i j}^{e,(\mathrm{~K}-1)}=-\int_{\Omega_{e}}\left[\left(\sum_{k=1}^{\mathrm{M}} \widetilde{u}_{1 k}^{(\mathrm{K}-1)}\left(\mathbf{a}_{k}\right) \phi_{k}\right)\left(\sum_{l=1}^{\mathrm{M}} \widetilde{u}_{2 l}^{(\mathrm{K}-1)}\left(\mathbf{a}_{l}\right) \frac{\partial \phi_{l}}{\partial x}\right)+\right. \\
\left.\left(\sum_{k=1}^{\mathrm{M}} \widetilde{u}_{2 k}^{(\mathrm{K}-1)}\left(\mathbf{a}_{k}\right) \phi_{k}\right)\left(\sum_{l=1}^{\mathrm{M}} \widetilde{u}_{2 l}^{(\mathrm{K}-1)}\left(\mathbf{a}_{l}\right) \frac{\partial \phi_{l}}{\partial y}\right)\right] \phi_{i} d \Omega
\end{array}
$$

El calculo de los vectores $\mathbf{k} 1^{(\mathrm{K}-1)}$ y $\mathbf{k} \mathbf{2}^{(\mathrm{K}-1)}$ se hace a nivel local (veáse apartado 2.5 ), $\mathrm{y}$ 
como $\left.\phi_{k}\right|_{\Omega_{e}}=\psi_{k}^{e}$, se usan las ecuaciones (2.2), (2.46) y (2.47) junto con la fórmula (3.4)-(3.5) para evaluar las integrales contenidas en (3.12). Observemos que la matriz asociada al sistema (3.11), es la matriz A definida en (2.23), entonces, como la factorización de Crout ya se hizo en el paso (i), lo único que se hace para resolver los sistemas del paso (ii) es aplicar la segunda etapa del método de Crout (3.9).

Se hicieron diferentes pruebas en las que se resolvieron numéricamente problemas de Navier-

Stokes estacionarios (no se tomó en cuenta el término temporal $\frac{\partial \mathrm{u}}{\partial t}$ ) usando un método de punto fijo. Por ejemplo, se resolvió numéricamente el ejemplo 4.1, dado en el capítulo 4, para una malla de 100 elementos usando diferentes números de Reynolds. Se encontró que este método. comienza a diverger para números de Reynolds mayores que 75 , además de que para Reynolds cercanos a 75 la convergencia es muy lenta (del orden de 1583 iteraciones).

Luego, una gran desventaja de usar el método de punto fijo es que su radio de convergencia depende del número de Reynolds, como lo confirma Karakashian [17] , ya que muestra que este método converge a una única solución siempre que se satisfaga la condición siguiente:

$$
\|\mathbf{f}\|_{-1}<\frac{L^{2} U^{2} C_{a}^{2}}{\operatorname{Re}^{2} C_{b}}
$$

en donde $C_{a}$ y $C_{b}$ son constantes que provienen de la $\mathbf{H}$ div-elípticidad de la forma bilineal $a(\cdot, \cdot)$ y de la continuidad del operador trilineal $a_{1}(\cdot, \cdot, \cdot)$, respectivamente.

Conforme el número de Reynolds se incremente será difícil que la condición (3.13) se satisfaga. Entonces, si se quieren hacer simulaciones sobre el comportamiento de diferentes fluidos viscosos, se recomienda incorporar a la modelación el término no estacionario $\frac{\partial \mathbf{u}}{\partial t}$, para que el radio de convergencia del método de punto fijo aumente como se exhibe en la sección siguiente.

\subsection{Problema no estacionario.}

Para resolver el problema no estacionario se utiliza un esquema implícito de Euler hacia atrás para discretizar la variable temporal y un esquema de punto fijo para resolver el "problema de Navier-Stokes estacionario" que se obtiene en cada iteración temporal, como se vio en el apartado 2.3 .

Esta propuesta se utiliza porque Temam [27] demuestra que el esquema implícito de Euler 
hacia atrás (descrito en el apartado 3.4.1) es incondicionalmente estable y que la solución permanece acotada en la norma $L^{2}$ independientemente de $\Delta t$ y de la viscosidad $\nu$. Además en Juárez-Valencia [16] se muestra que el método iterativo descrito en el apartado 3.4.2, tiene un punto fijo si se cumple que:

$$
\Delta t<\frac{\nu}{\mathrm{U}^{2}}
$$

en donde

$$
\mathrm{U} \geq \max \left\{\left\|\mathbf{u}_{h}^{(\mathrm{N}, \mathrm{K}-1)}\right\|_{\infty},\left\|\mathbf{u}_{h}^{(\mathrm{N})}\right\|_{\infty}\right\}
$$

(obsérvese que $\mathbf{u}_{h}^{(\cdot \cdot \cdot)}$ está definida enseguida en $(3.16)-(3.17)$ ). Es decir, en la práctica basta con pedir que

$$
\Delta t \approx \frac{1}{\operatorname{Re}}
$$

para asegurar convergencia. (En las escalas usuales $U=1$ y $L=1$ ).

\subsubsection{Esquema implícito de Euler hacia atrás.}

Dado $\mathbf{u}_{h}^{(0)}$. Iterar para $\mathrm{N}=1,2,3, \ldots \ldots$, lo siguiente

$$
\begin{array}{cc}
\frac{1}{\Delta t}\left(\mathbf{u}_{h}^{(\mathrm{N})}, \mathbf{v}_{h}\right)+a_{1}\left(\mathbf{u}_{h}^{(\mathbb{N})} ; \mathbf{u}_{h}^{(\mathbb{N})}, \mathbf{v}_{h}\right)+a\left(\mathbf{u}_{h}^{(\mathrm{N})}, \mathbf{v}_{h}\right)+b\left(p_{h}^{(\mathbb{N})}, \mathbf{v}_{h}\right)= \\
L_{f}^{(\mathbb{N})}\left(\mathbf{v}_{h}\right)+\frac{1}{\Delta t}\left(\mathbf{u}_{h}^{(\mathbb{N}-1)}, \mathbf{v}_{h}\right) & \forall \mathbf{v}_{h} \in \mathbf{V}_{h}^{0}, \\
b\left(q_{h}, \mathbf{u}_{h}^{(\mathbb{N})}\right)=0 & \forall q_{h} \in Q_{h}^{0} .
\end{array}
$$

hasta que $\frac{\left\|\mathbf{u}_{h}^{(\mathrm{I})}-\mathbf{u}_{h}^{(\mathrm{N}-1)}\right\|_{0}}{\Delta t\left\|\mathbf{u}_{h}^{(\mathrm{N})}\right\|_{0}} \leq 10^{-6} \quad$ o $\quad \mathrm{N}>$ MAXIT2.

Observemos que (3.15) es una clase de "sistemas de ecuaciones de Navier-Stokes estacionarias".

\subsubsection{Esquema de Punto Fijo.}

El esquema de Punto Fijo que se utiliza para resolver el sistema de ecuaciones (3.15) es:

(i) Asumiendo que se conoce $\mathbf{u}_{h}^{(\mathrm{N}-1)}$ y $p_{h}^{(\mathrm{N}-1)}$, definimos

$$
\mathbf{u}_{h}^{(\mathrm{N}, 0)}:=\mathbf{u}_{h}^{(\mathrm{N}-1)} \quad \mathrm{y} \quad p_{h}^{(\mathrm{N}, 0)}:=p_{h}^{(\mathrm{N}-1)}
$$


(ii) Para $\mathrm{K}=1,2,3, \ldots \ldots$, resolver

$$
\begin{gathered}
\frac{1}{\Delta t}\left(\mathbf{u}_{h}^{(\mathrm{N}, \mathrm{K})}, \mathbf{v}_{h}\right)+a\left(\mathbf{u}_{h}^{(\mathrm{N}, \mathrm{K})}, \mathbf{v}_{h}\right)+b\left(p_{h}^{(\mathrm{N}, \mathrm{K})}, \mathbf{v}_{h}\right)=L_{f}^{(\mathrm{N})}\left(\mathbf{v}_{h}\right)+ \\
\frac{1}{\Delta t}\left(\mathbf{u}_{h}^{(\mathrm{N}-1)}, \mathbf{v}_{h}\right)+a_{1}\left(\mathbf{u}_{h}^{(\mathrm{N}, \mathrm{K}-1)} ; \mathbf{u}_{h}^{(\mathrm{N}, \mathrm{K}-1)}, \mathbf{v}_{h}\right) \quad \forall \mathbf{v}_{h} \in \mathbf{V}_{h}^{0}, \\
b\left(q_{h}, \mathbf{u}_{h}^{(\mathrm{N}, \mathrm{K})}\right)=0 \quad \forall q_{h} \in Q_{h}^{0}
\end{gathered}
$$

hasta que $\frac{\left\|\mathbf{u}_{h}^{(\mathrm{N}, \mathrm{K})}-\mathbf{u}_{h}^{(\mathrm{N}, \mathrm{K}-1)}\right\|_{0}}{\left\|\mathbf{u}_{h}^{(\mathrm{N}, \mathrm{K})}\right\|_{0}} \leq 10^{-8} \quad$ o $\quad \mathrm{N}>$ MAXIT1.

Se observa de (3.17) que en cada paso del tiempo (N) se resuelve un problema cuasi-Stokes. Una ventaja es que en su implantación sólo se usa (3.9), ya que la factorización de Crout se hace una vez, cuando se inicia el método para calcular $\mathbf{u}_{h}^{(0,0)}$, en donde se resuelve un problema cuasi-Stokes sin considerar al término $\frac{1}{\Delta t}\left(\mathbf{u}_{h}^{(N-1)}, \mathbf{v}_{h}\right)$.

\subsection{Descripción del Programa NAVSTOKE}

El programa NAVSTOKE es un código que resuelve numéricamente por medio de la técnica de Elemento Finito sobre regiones no estándares el problema (0.1)-(0.2) definido en la introducción, o bien, los problemas estacionarios de Stokes o Navier-Stokes. La estructura del programa se da en 3.5.2.

Una parte importante para que el programa comience a trabajar es dar todos los datos de la malla del dominio de estudio. El modo de construir estos datos se explica a continuación.

\subsubsection{Programas que discretizan el dominio $\bar{\Omega}$.}

Por un momento nos referiremos al dominio $\bar{\Omega}$ del ejemplo 4.1 del capítulo 4 . Se trata de un rectángulo de una unidad de largo por una unidad de ancho y es mallado utilizando las subrutinas COMEFI y GENNOD que forman parte del Paquete Malla creado por P. Saavedra [23].

La subrutina COMEFI cuadricula el dominio $\bar{\Omega}$ que se muestra en la Figura 4-1 . La subrutina GENNOD ocupa los datos de la malla rectangular para generar los nodos o grados de libertad de cada elemento $\Omega_{e}$ y crea la estructura de datos que requiere el programa NAVSTOKE. 
La forma del dominio $\bar{\Omega}$ que se usa en el ejemplo 4.2 se exhibe en la Figura 4-10. Para mallar este dominio fue necesario crear un programa exclusivo para dicho fin, el cual se llama MALCILI.

El programa MALCILI (elaborado por la autora de este libro), subdivide la región $\bar{\Omega}$ en cuadriláteros con dos lados curvos como se ve en la Fig. 4-10. Para construir estos cuadriláteros primero se hizo coincidir el origen de los ejes coordenados con el centro del cilindro. Se dividió tanto el semicuadrado superior (la curva exterior) como el semicírculo superior (el que representa al cilindro) en igual número de partes. Se formaron rayos con la unión de las divisiones de ambas gráficas y se intersectaron con cada una de las curvas siguientes:

$$
\left(\frac{x}{a_{n}}\right)^{2}+\left(\frac{y}{b_{n}}\right)^{n}=1, \quad \text { para } \quad n=3,4,5,6,8,10,12,14,16
$$

Después se reflejó la región con respecto al eje horizontal y se aplicó una transformación que contrae a la región. De este modo se generan los nodos de la presión y sólo se aumentan los puntos medios de cada cuadrilátero para obtener los nodos de la velocidad.

Hay que mencionar que ambos programas forman cuadriláteros respetando las propiedades (i)-(vi) descritas en el apartado 2.1. Además, la estructura de datos de salida que tienen es la que necesita NAVSTOKE y se da a continuación:

- Nombre del proyecto.

- Nombre del autor.

- Fecha.

- Número de vértices en $x$, número de vértices en $y$, número de nodos de la velocidad, número de elementos, número de grados de frontera libre, número de nodos de la presión.

- La numeración global de los nodos de la velocidad para cada elemento.

- La numeración global de los nodos de la presión para cada elemento.

- Número de referencia y coordenadas de los nodos de la velocidad. 


\subsubsection{Estructura del código NAVSTOKE.}

En la Fig. 3-1 se muestra el esquema del programa principal del código NAVSTOKE. A continuación describiremos las subrutinas más importantes a las que llama:

- PIDEDATO.

Lectura de datos iniciales, los cuales son:

Nombre del archivo de entrada con los datos de la malla.

Nombre del archivo de impresión con los datos de salida.

Número de Reynolds, número de iteraciones para el método de punto fijo, tolerancia.

Incremento del tiempo, número de iteraciones para el tiempo, tolerancia.

Tipo de norma: $\mathbf{L}_{\infty}$ o $\mathbf{L}_{2}$.

Valor de la presión y número de nodo en donde se fija.

Si se iniciar con Stokes o con un archivo de datos.

El nivel de impresión

- RECUPER.

Lectura de datos de la malla que se creó con cualquiera de los programas descritos en el apartado 3.5.1.

- CUADGAUS.

Evalúa los puntos de integración $\left(\xi_{l}, \eta_{l}\right)$ y los pesos de integración $w_{l}$ dados en la tabla (3.5) .

- FORMAPRE.

Calcula los valores de las funciones base locales para la presión $\left(\hat{Q}_{1}\left(\Omega_{\hat{e}}\right)\right)$ y de sus derivadas en el punto de integración $\left(\xi_{l}, \eta_{l}\right)$. Dichas funciones están definidas en (2.1) y sus derivadas parciales son las siguientes: 


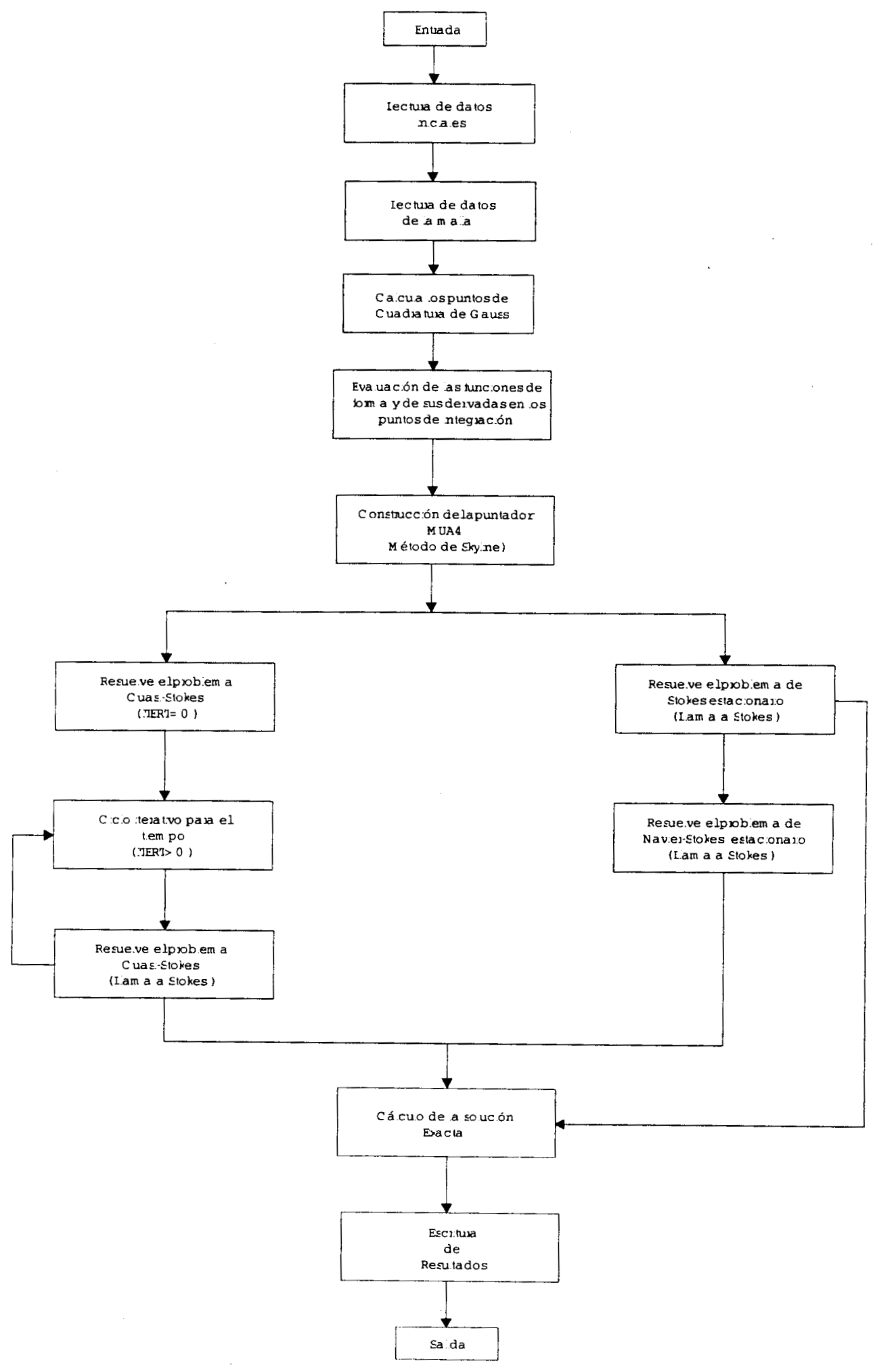

Figura 3-1: Esquema del programa principal de NAVSTOKE. 


$$
\begin{array}{ll}
\frac{\partial \widehat{\varphi}_{1}(\xi, \eta)}{\partial \xi}=-\frac{1}{4}(1-\eta), & \frac{\partial \widehat{\varphi}_{1}(\xi, \eta)}{\partial \eta}=-\frac{1}{4}(1-\xi), \\
\frac{\partial \widehat{\varphi}_{2}(\xi, \eta)}{\partial \xi}=\frac{1}{4}(1-\eta), & \frac{\partial \widehat{\varphi}_{2}(\xi, \eta)}{\partial \eta}=-\frac{1}{4}(1+\xi), \\
\frac{\partial \widehat{\varphi}_{3}(\xi, \eta)}{\partial \xi}=\frac{1}{4}(1+\eta), & \frac{\partial \widehat{\varphi}_{3}(\xi, \eta)}{\partial \eta}=\frac{1}{4}(1+\xi), \\
\frac{\partial \widehat{\varphi}_{4}(\xi, \eta)}{\partial \xi}=-\frac{1}{4}(1+\eta), & \frac{\partial \widehat{\varphi}_{4}(\xi, \eta)}{\partial \eta}=\frac{1}{4}(1-\xi) .
\end{array}
$$

\section{- FORMAVEL.}

Calcula los valores de las funciones base locales para $\hat{\mathrm{Q}}_{2}\left(\Omega_{\hat{e}}\right)$ en el punto de integración $\left(\xi_{l}, \eta_{l}\right)$, estas funciones se definieron en (2.2). También evalúa sus derivadas parciales con respecto a $\xi$ y a $\eta$ en los nueve puntos de integración. Las derivadas parciales son las siguientes:

$$
\begin{array}{ll}
\frac{\partial \hat{\psi}_{1}(\xi, \eta)}{\partial \xi}=\left(\frac{\xi}{2}-\frac{1}{4}\right)\left(\eta^{2}-\eta\right), & \frac{\partial \hat{\psi}_{1}(\xi, \eta)}{\partial \eta}=\left(\xi^{2}-\xi\right)\left(\frac{\eta}{2}-\frac{1}{4}\right), \\
\frac{\partial \hat{\psi}_{2}(\xi, \eta)}{\partial \xi}=\left(\frac{\xi}{2}+\frac{1}{4}\right)\left(\eta^{2}-\eta\right), & \frac{\partial \hat{\psi}_{2}(\xi, \eta)}{\partial \eta}=\left(\xi^{2}+\xi\right)\left(\frac{\eta}{2}-\frac{1}{4}\right), \\
\frac{\partial \hat{\psi}_{3}(\xi, \eta)}{\partial \xi}=\left(\frac{\xi}{2}+\frac{1}{4}\right)\left(\eta^{2}+\eta\right), & \frac{\partial \hat{\psi}_{3}(\xi, \eta)}{\partial \eta}=\left(\xi^{2}+\xi\right)\left(\frac{\eta}{2}+\frac{1}{4}\right), \\
\frac{\partial \hat{\psi}_{4}(\xi, \eta)}{\partial \xi}=\left(\frac{\xi}{2}-\frac{1}{4}\right)\left(\eta^{2}+\eta\right), & \frac{\partial \hat{\psi}_{4}(\xi, \eta)}{\partial \eta}=\left(\xi^{2}-\xi\right)\left(\frac{\eta}{2}+\frac{1}{4}\right), \\
\frac{\partial \hat{\psi}_{5}(\xi, \eta)}{\partial \xi}=(-\xi)\left(\eta^{2}-\eta\right), & \frac{\partial \hat{\psi}_{5}(\xi, \eta)}{\partial \eta}=\left(1-\xi^{2}\right)\left(\eta-\frac{1}{2}\right), \\
\frac{\partial \hat{\psi}_{6}(\xi, \eta)}{\partial \xi}=\left(\xi+\frac{1}{2}\right)\left(1-\eta^{2}\right), & \frac{\partial \hat{\psi}_{6}(\xi, \eta)}{\partial \eta}=-\eta\left(\xi^{2}+\xi\right), \\
\frac{\partial \hat{\psi}_{7}(\xi, \eta)}{\partial \xi}=(-\xi)\left(\eta^{2}+\eta\right), & \frac{\partial \hat{\psi}_{7}(\xi, \eta)}{\partial \eta}=\left(1-\xi^{2}\right)\left(\eta+\frac{1}{2}\right), \\
\frac{\partial \hat{\psi}_{8}(\xi, \eta)}{\partial \xi}=\left(\xi-\frac{1}{2}\right)\left(1-\eta^{2}\right), & \frac{\partial \hat{\psi}_{8}(\xi, \eta)}{\partial \eta}=-\eta\left(\xi^{2}-\xi\right), \\
\frac{\partial \hat{\psi}_{9}(\xi, \eta)}{\partial \xi}=-2 \xi\left(1-\eta^{2}\right), & \frac{\partial \hat{\psi}_{9}(\xi, \eta)}{\partial \eta}=-2 \eta\left(1-\xi^{2}\right) .
\end{array}
$$

\section{- PUPERFIL.}

Una pequeña modificación al algoritmo del método de perfil descrito en [22] fue implementada en esta subrutina. Esta adaptación consiste en poner "unos" sobre la diagonal de los renglones que esten asociados a condiciones de Dirichlet homogéneas y no homogéneas. Básicamente aquí se construye el vector MUA4 y se calcula el tamaño del vector GA.

\section{- STOKES.}

Esta subrutina es muy importante porque construye las matrices locales, las ensambla y resuelve el sistema lineal del problema de Stokes o de cuasi-Stokes, por 
lo que en la Fig. 3-2 se describe su estructura.

Las subrutinas que forman parte de la subrutina STOKES son las siguientes:

- VECRESTR.

Construye el vector de restricciones VRES $_{\mathbf{m} \times 1}$, el cual contiene los valores de la velocidad en los nodos de la frontera donde exista una condición de frontera tipo Dirichlet no homogénea, es decir, tanto VRES como GB almacenan en el renglón $j$-ésimo el valor de $\widetilde{g}_{1}\left(\mathbf{a}_{j}\right)$ y en el renglón $\mathrm{M}+j$ el valor de $\widetilde{g}_{2}\left(\mathbf{a}_{j}\right) . \mathrm{GB}_{\mathrm{m} \times 1}$ es un arreglo que representa al vector global b. Además fija el valor de la presión $\widetilde{g}_{\mathrm{P}}(\mathrm{ap})$ en el nodo elegido por el usuario.

- CONSTIFF.

Arma la matriz local $\overline{\mathbf{A}}^{e}$ definida en (2.31)-(2.34) y se le llama STIFF $_{22 \times 22}$. Si ISTOLIK=1, STIFF es la matriz local asociada al problema de cuasi-Stokes, de lo contrario STIFF es la matriz local asociada al problema de Stokes.Dado que se utiliza la fórmula de integración (3.4), se calculan para cada punto de integración $\left(\xi_{l}, \eta_{l}\right)$ los valores de todas las componentes de $\overline{\mathbf{A}}^{e}$ y se incrementan estos a los valores de las componentes ya calculados. Para ello, se ocupan las subrutinas TRANFDERV y VECONEX. Por otro lado, la aplicación de las condiciones de Dirichlet homogéneas se hace directamente en esta rutina, en la cual a través del vector de enlace LM se localizan los nodos que pertenecen a la frontera con estas condiciones, para después bloquear los renglones o columnas correspondientes (veáse subrutina VECONEX).

- TRANFDERV.

Calcula las derivadas $\frac{\partial \psi_{i}^{e}}{\partial x}, \frac{\partial \psi_{i}^{e}}{\partial y}, \frac{\partial \hat{\varphi}_{l}^{e}}{\partial x}$ y $\frac{\partial \widehat{\varphi}_{1}^{e}}{\partial y}$ usando las ecuaciones (2.46)-(2.47) y el factor de integración dado por $\left|\mathbf{J}\left(\xi_{l}, \eta_{l}\right)\right| w_{l}$ (veáse ec. (3.4)).

- VECONEX.

Forma los vectores $\mathrm{LM}_{22 \times 1}$ y $\mathrm{RESCERO} \mathrm{C}_{2 \times 1}$ que continen la información necesaria para ensamblar y bloquear las matrices locales. Los primeros 9 renglones contienen los datos sobre la primera componente de la velocidad, los siguientes 9 


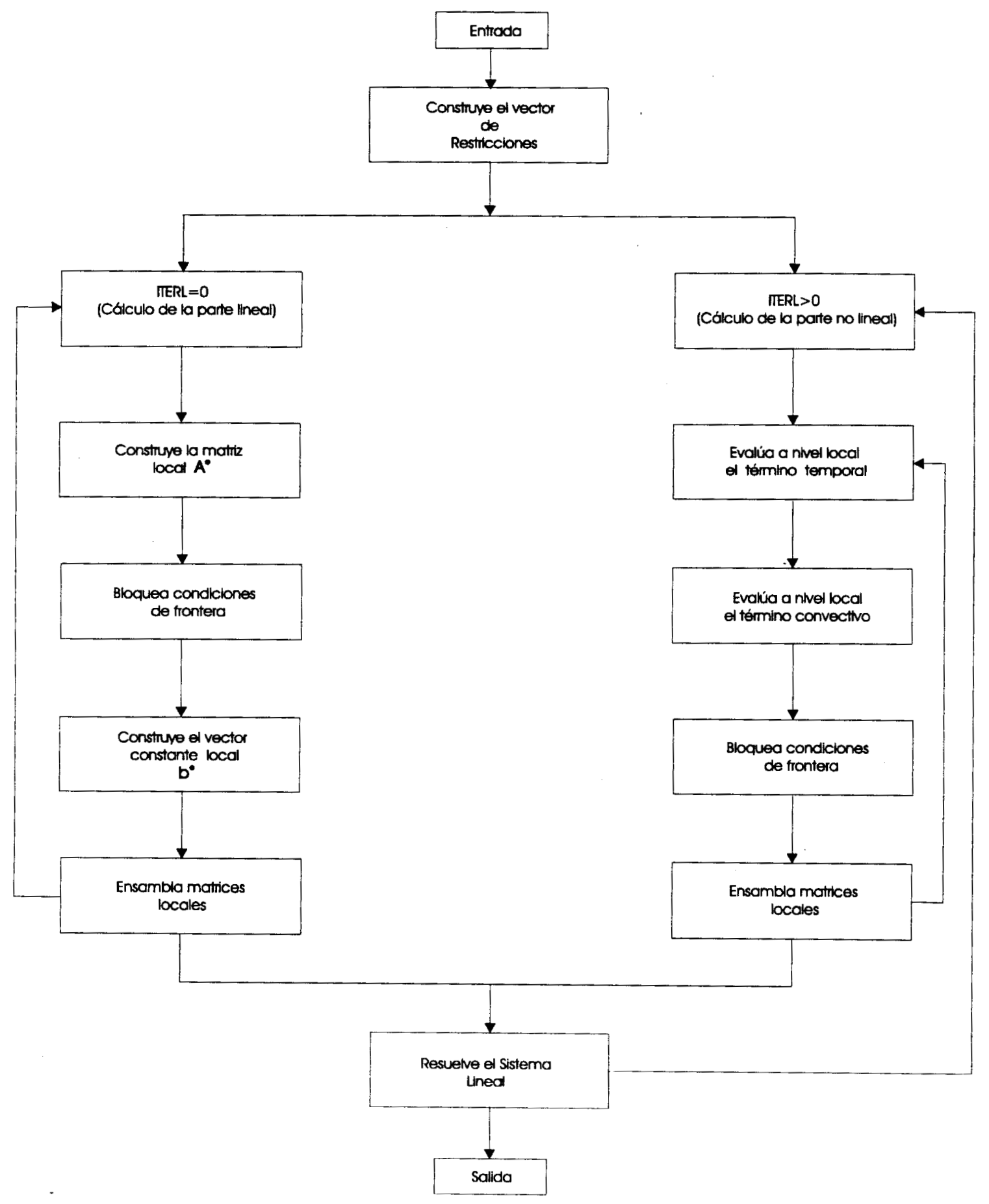

Figura 3-2: Esquema de la rutina STOKES. 
renglones tienen la información de la segunda componente de la velocidad y los 4 restantes los datos de la presión. En general los vectores satisfacen lo siguiente:

- Si $L M(I)=N$ el renglón I de la matriz STIFF contribuye al renglón $\mathrm{N}$ de la matriz global GA.

- Si $\mathrm{LM}(\mathrm{I})=0$ indica que el grado de libertad relacionado con el reglón I tiene una condición de frontera igual a cero. El renglón $\mathrm{N}$ global se almacena en el renglón $\mathrm{I}$ del vector $\mathrm{RESCERO}(\mathrm{I})=\mathrm{N}$.

- Si $L M(I)=-N$ indica que el grado de libertad relacionado con el reglón I tiene una condición de frontera distinta de cero. Este valor es almacenado en el vector VRES en la posición $\mathrm{N}$, es decir, $\operatorname{VRES}(\mathrm{N})=\mathrm{U}$ (el vector VRES está descrito en la subrutina VECRESTR).

\section{- VECONST.}

Construye el vector local $\bar{b}^{e}$ definido en (2.35)-(2.36) y almacenado en $\mathrm{BE}_{22 \times 1}$, usando la información de los arreglos VRES y STIFF si ITERL=0; sino BE guarda el término convectivo y/o el término temporal.

- NAVIER.

Forma un vector local $\mathrm{BENL}_{22 \times 1}$, el cual contiene la parte no lineal $\mathbf{k} \mathbf{1}^{(\mathrm{K}-1)} \mathrm{y}$ $\mathbf{k 2}^{(K-1)}$ definidas en (3.12). Se hacen los calculos a nivel local.

- FZAVOL.

Construye el vector local $\mathrm{FVOL}_{22 \times 1}$, el cual está formado por la parte de la fuerza volumétrica.

- FZALOC.

Almacena en el vector local $\mathrm{FL}_{22 \times 1}$, la parte correspondiente al término $\frac{1}{\Delta t}\left(\mathbf{u}_{h}^{(\mathrm{N}-1)}, \mathbf{v}_{h}\right)$.

- ENSAMBLE.

Para formar la matriz global $\mathbf{A}$, se calculan una por una las matrices locales $\overline{\mathbf{A}}^{e}$ para cada $\Omega_{e} \in \boldsymbol{\Omega}_{h}$ definidas en (2.31)-(2.34) y se guarda dicha información en la matriz elemental $\mathbf{A}^{e}$ correspondiente. Después se suman estas matrices a las contribuciones de cada elemento de acuerdo al apartado 2.5. Análogamente, se 
calcula el vector constante $\mathbf{b}$. Al proceso anterior se le llama ensamble de $\mathbf{A}$ y b, y es implementado en esta rutina con ayuda del vector de enlace LM.

- CROUT.

Factoriza la matriz $\mathbf{A}$ usando el método de Crout adaptado para el método de perfil, es decir, $\mathbf{A}=\mathbf{L} \mathbf{D}^{t} \mathbf{L}$. En el vector $\mathbf{G A}$ se almacenan las entrada de las matrices $\mathbf{L D}$ y $\mathbf{D}$.

- RESLDtL.

Esta rutina realiza la segunda etapa del método de Crout, es decir hace los cálculos correspondientes a $(3.9)$

- calerror.

Calcula el error relativo para la velocidad y la presión. Para ello se ayuda de la rutina CALNORMA, que evalúa la norma $\mathbf{L}_{\infty}$ o $\mathbf{L}_{2}$ de un vector.

En este capítulo se expusieron las razones por las que se usa una fórmula de cuadratura de Gauss de 9 puntos para resolver las integrales, el método de Crout adaptado para el método de perfil (skyline) para resolver el sistema de ecuaciones lineales y el método iterativo de punto fijo para resolver los sistemas no lineales. Además se han mencionado las condiciones para que el esquema implícito de Euler hacia atrás sea estable y para que el método iterativo de punto fijo sea convergente. Finalmente, se ha presentado una breve descripción del programa NAVSTOKE el cual se validó con varios ejemplos, pero solamente se presentarán en el capítulo siguiente los más interesantes. 


\section{Capítulo 4}

\section{Ejemplos Numéricos}

En este capitulo se resuelven numéricamente con el programa NAVSTOKE dos problemas clásicos de flujo de fluidos que normalmente aparecen en la literatura como problemas de prueba (benchmark) . El dominio del primer problema se refiere a una cavidad cuadrada, para el cual se resuelve numéricamente el problema de Stokes y Navier-Stokes. Con los resultados numéricos obtenidos se realizan curvas de convergencia del elemento finito utilizado (veánse ecs. 2.19). El segundo ejemplo es un problema de un flujo de un fluido viscoso que pasa sobre un cilindro cerca de una pared en movimiento. En este caso se resuelve el problema de Stokes en dicho dominio, el cual es no trivial y presenta una frontera curva (el cilindro). Para resolver este problema es necesario usar elementos isoparamétricos.

\subsection{Aplicación al flujo en una cavidad cuadrada.}

Este ejemplo es presentado en la literatura como un problema prueba (veánse Shih-Tan-Hwang [25] y Klouček-Rys [18]). Consiste en una cavidad cuadrada que contiene un fluido cuyo flujo recircula, como se advierte en la Fig. 4-1.

La forma de modelar este problema es a través de las ecuaciones de Navier-Stokes y si el fluido es muy viscoso se puede modelar como un problema de Stokes como se detalla a continuación. 


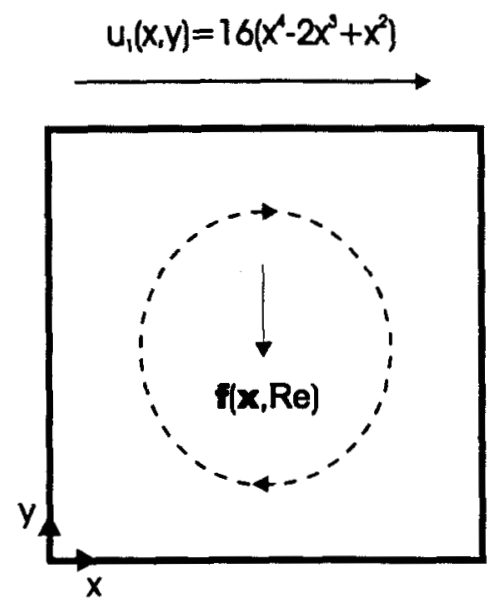

Figura 4-1: Problema de la cavidad cuadrada.

\subsubsection{Problema de Stokes.}

Si el fluido es muy viscoso y su flujo es bidimensional, estacionario, homogéneo, isotérmico e incompresible, entonces el problema de la cavidad cuadrada en este caso se puede modelar por medio de las ecuaciones de Stokes:

$$
\begin{aligned}
& -\frac{1}{\operatorname{Re}} \Delta \mathbf{u}(\mathbf{x})+\nabla p(\mathbf{x}, \operatorname{Re})=\mathbf{f}(\mathbf{x}, \operatorname{Re}), \quad \forall \mathbf{x} \in[0,1] \times[0,1], \\
& \nabla \cdot \mathbf{u}(\mathbf{x})=0, \quad \forall \mathbf{x} \in[0,1] \times[0,1],
\end{aligned}
$$

junto con las condiciones de frontera:

$$
\begin{gathered}
\mathbf{u}(0, y)=\mathbf{u}(1, y)=0 \quad \forall y \in[0,1] ; \quad \mathbf{u}(x, 0)=0 \quad \forall x \in[0,1] \\
u_{1}(x, 1)=16\left(x^{4}-2 x^{3}+x^{2}\right) \quad \text { y } \quad u_{2}(x, 1)=0 \quad \forall x \in[0,1]
\end{gathered}
$$

donde $\mathbf{x}=(x, y), \mathbf{u}=\left(u_{1}, u_{2}\right), \mathbf{f}=\left(f_{1},-f_{2}\right)$ tal que

$$
\begin{aligned}
& f_{1}(x, y, \operatorname{Re})=0 \quad \text { y } \\
& f_{2}(x, y, \operatorname{Re})=-\frac{8}{\operatorname{Re}}\left[f^{\prime \prime \prime}(x) g(y)+2 f^{\prime}(x) g^{\prime \prime}(y)+24 F(x)\right]
\end{aligned}
$$

con 


$$
\begin{aligned}
& f(x)=x^{2}(x-1)^{2}, \\
& F(x)=\int_{0}^{x} f(x) d x=\frac{1}{5} x^{5}-\frac{1}{2} x^{4}+\frac{1}{3} x^{3}, \\
& f^{\prime}(x)=4 x^{3}-6 x^{2}+2 x, \\
& f^{\prime \prime}(x)=12 x^{2}-12 x+2, \\
& f^{\prime \prime \prime}(x)=24 x-12, \\
& g(y)=y^{2}\left(y^{2}-1\right) \\
& g^{\prime}(y)=4 y^{3}-2 y \\
& g^{\prime \prime}(y)=12 y^{2}-2, \\
& g^{\prime \prime \prime}(y)=24 y .
\end{aligned}
$$

\section{Solución analítica.}

La solución analítica para este problema es:

$$
\begin{aligned}
& u_{1}(x, y)=8 f(x) g^{\prime}(y) \\
& u_{2}(x, y)=-8 f^{\prime}(x) g(y) \\
& p(\mathbf{x}, \operatorname{Re})=-\frac{8}{\operatorname{Re}}\left[f^{\prime}(x) g^{\prime}(y)+F(x) g^{\prime \prime \prime}(y)\right]
\end{aligned}
$$

en donde las funciones $F(x), f(x)$ y $g(y)$, así como sus derivadas están definidas en (4.4). Observemos que la condición $\int_{\Gamma} \mathbf{g} \cdot \mathbf{n} d \Omega=0$ se cumple, ya que $\mathbf{g}(x, y)=\left(u_{1}(x, y), u_{2}(x, y)\right)$ representa la condición de frontera y $\mathbf{n}$ el vector normal en cada punto $(x, y)$ de la frontera de la cavidad.

También observemos que la fuerza total ejercida sobre el fluido es

$$
\mathcal{F}=\mathbf{F}_{v i s \cos a}+\mathbf{f}=\left(f_{1}-T_{y x}\right) \mathbf{i}+\left(-f_{2}-T_{y y}\right) \mathbf{j}
$$

en donde $T_{y x}$ y $T_{y x}$ son las componentes del tensor de esfuerzos $\mathbf{T}$ (veáse ec. (1.24)) definidas por

$$
\begin{aligned}
& T_{y y}=\frac{8}{\operatorname{Re}}\left[-f^{\prime}(x) g^{\prime}(y)+F(x) g^{\prime \prime \prime}(y)\right] \quad y \\
& T_{y x}=\frac{8}{\operatorname{Re}}\left[-f^{\prime \prime}(x) g(y)+f(x) g^{\prime \prime}(y)\right]
\end{aligned}
$$


Luego, la fuerza total sobre $y=0$ en la dirección $y$ es:

$$
\mathcal{F}_{y}(x, 0)=-f_{2}(x, 0, \operatorname{Re})-T_{y y}(x, 0)=\frac{192}{\operatorname{Re}} F(x)
$$

Análogamente, la fuerza total sobre $y=0.5$ en la dirección $y$ es:

$$
\mathcal{F}_{y}(x, 0.5)=-f_{2}(x, 0.5, \operatorname{Re})-T_{y y}(x, 0.5)=\frac{3}{\operatorname{Re}}\left[4 f^{\prime}(x)-\frac{1}{2} f^{\prime \prime \prime}(x)+32 F(x)\right]
$$

De lo anterior se puede concluir que el flujo del fluido contenido sobre la cavidad recircula debido a la fuerza de corte y a la fuerza volumétrica.

\section{Resultados numéricos。}

Se resolvió el problema de Stokes para $R e=1$ en diferentes mallas. Las mallas utilizadas son uniformes y contienen 100, 400, 625 y 1600 elementos, es decir, el parámetro de discretización $h$ es igual a $0.1,0.05,0.04$ y 0.025 respectivamente.

En la Fig. 4-2 se muestran los campos de velocidades tanto para la solución aproximada como para la solución exacta del problema descrito en (4.1)-(4.4) para $R e=1$ y para la mallas con $h$ igual a 0.1. En ella se ve claramente como la fuerza de corte ejercida en la parte superior de la cavidad cuadrada, provoca la recirculación del flujo.

En la Fig. 4-3 se exhiben los contornos de presión para una malla de 400 elementos $(h=0.05)$ tanto para la solución aproximada como para la exacta. El nodo en el que se fijó el valor de la presión tiene coordenadas $(0,0)$ y el valor que se le dió fue de cero en todas las corridas. El valor más alto de la presión se alcanzó en el punto $(1,1)$ con un valor de 6.40 para $R e=1$, que era lo que se esperaba como se ve en la solución analítica dada en (4.5). En dicha figura mientras más claro sea el tono de gris, más alto será el valor de la presión.

\begin{tabular}{|c|c|c|c|}
\hline \multicolumn{2}{|c|}{ Velocidad } & \multicolumn{2}{c|}{ Presión } \\
\hline$h$ & Error Relativo & $h$ & Error Relativo \\
\hline 0.1 & 0.00353435 & 0.1 & 0.0290371 \\
0.05 & 0.00353863 & 0.05 & 0.0259648 \\
\hline
\end{tabular}

Tabla 4-1: Comparación entre la solución numérica del problema de Stokes $\mathbf{u}_{S}$ con $\operatorname{Re}=1$ para la cavidad cuadrada y la solución exacta del problema de Navier-Stokes $\mathbf{u}_{E N S}$. 

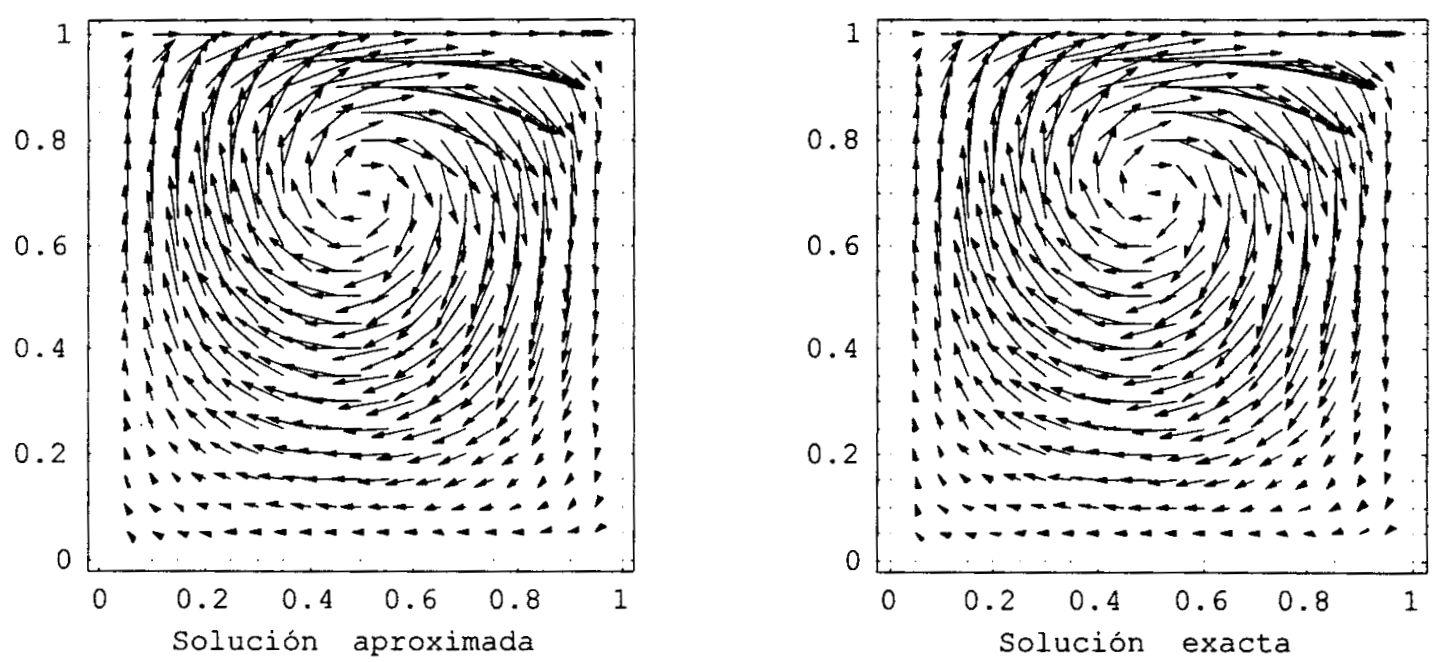

Figura 4-2: Campos de velocidades para el problema de Stokes. $R e=1, h=0.1$.
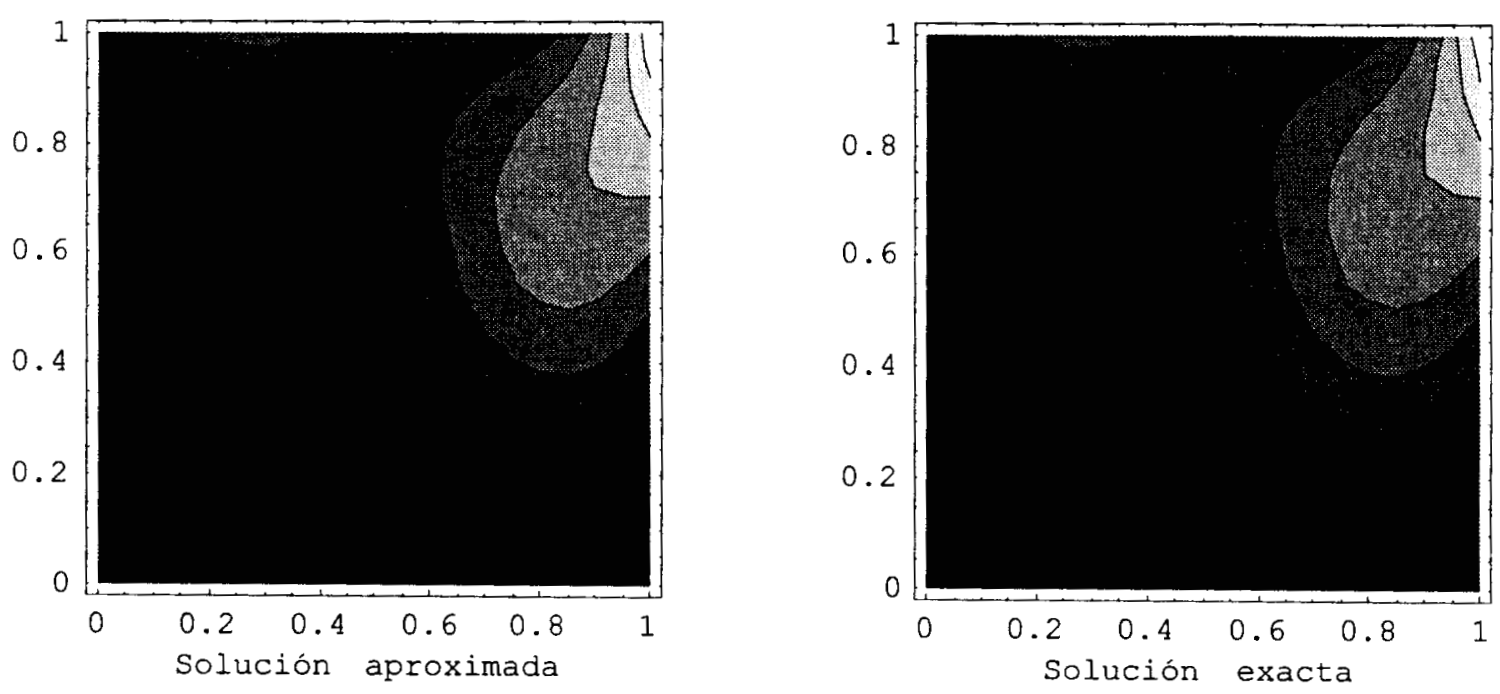

Figura 4-3: Contornos de presión para el problema de Stokes. $R e=1, h=0.05$. 
Con objeto de verificar que también se modela bien este problema $(R e=1)$ con las ecuaciones de Stokes, se comparó esta solución con la solución exacta de las ecuaciones de Navier-Stokes (veáse sección 4.1.2) en diferentes mallas. En la Tabla 4-1 se muestran las diferencias relativas para $h=0.1,0.05$. La diferencia relativa está dada por $\frac{\left\|\mathbf{u}_{S}-\mathbf{u}_{E N S}\right\|_{0}}{\left\|\mathbf{u}_{E N S}\right\|_{0}}$. En dicha tabla puede observarse que el mayor porcentaje del error para el cálculo de la velocidad es de $0.35 \%$, y para el cálculo de la presión de 3\% aproximadamente. Es de esperarse que estos porcentajes sean menores entre menor es Re, sin embargo en este trabajo no se hicieron comparaciones para soluciones numéricas del problema de Stokes con Re menores que 1.

\subsubsection{Problema de Navier-Stokes.}

Consideremos ahora el problema de Navier-Stokes para la cavidad cuadrada. Si el flujo es bidimensional, estacionario, homogéneo, isotérmico e incompresible, las ecuaciones que lo modelan son:

$$
\begin{gathered}
\mathbf{u} \cdot \boldsymbol{\nabla} \mathbf{u}-\frac{1}{\operatorname{Re}} \Delta \mathbf{u}(\mathbf{x})+\nabla p(\mathbf{x}, \operatorname{Re})=\mathbf{f}(\mathbf{x}, \operatorname{Re}), \quad \forall \mathbf{x} \in[0,1] \times[0,1], \\
\boldsymbol{\nabla} \cdot \mathbf{u}(\mathbf{x})=0, \quad \forall \mathbf{x} \in[0,1] \times[0,1]
\end{gathered}
$$

junto con las condiciones de frontera:

$$
\begin{gathered}
\mathbf{u}(0, y)=\mathbf{u}(1, y)=0 \quad \forall y \in[0,1] ; \quad \mathbf{u}(x, 0)=0 \quad \forall x \in[0,1] \\
u_{1}(x, 1)=16\left(x^{4}-2 x^{3}+x^{2}\right) \quad \text { y } \quad u_{2}(x, 1)=0 \quad \forall x \in[0,1]
\end{gathered}
$$

en donde $\mathbf{x}=(x, y), \mathbf{u}=\left(u_{1}, u_{2}\right)$. El lado derecho $\mathbf{f}=\left(f_{1},-f_{2}\right)$ en (4.6) es tal que

$$
\begin{array}{cl}
f_{1}(x, y, \mathrm{Re})= & 0 \quad \mathrm{y} \\
f_{2}(x, y, \mathrm{Re})=-\frac{8}{\operatorname{Re}}\left[24 F(x)+2 f^{\prime}(x) g^{\prime \prime}(y)+f^{\prime \prime \prime}(x) g(y)\right] \\
\\
-64\left[F_{2}(x) G(x)-g(y) g^{\prime}(y) F_{1}(x)\right]
\end{array}
$$

con 


$$
\begin{array}{ll}
f(x)=x^{2}(x-1)^{2}, & f^{\prime}(x)=4 x^{3}-6 x^{2}+2 x, \\
f^{\prime \prime}(x)=12 x^{2}-12 x+2, & f^{\prime \prime \prime}(x)=24 x-12, \\
g(y)=y^{2}\left(y^{2}-1\right), & g^{\prime}(y)=4 y^{3}-2 y, \\
g^{\prime \prime}(y)=12 y^{2}-2, & g^{\prime \prime \prime}(y)=24 y \\
F(x)=\int_{0}^{x} f(x) d x=\frac{1}{5} x^{5}-\frac{1}{2} x^{4}+\frac{1}{3} x^{3} \\
F_{1}(x)=f(x) f^{\prime \prime}(x)-\left[f^{\prime}(x)\right]^{2} \\
F_{2}(x)=\int_{0}^{x} f(x) f^{\prime}(x) d x=\frac{1}{2}[f(x)]^{2} \\
G(x)=g(y) g^{\prime \prime \prime}(y)-g^{\prime}(y) g^{\prime \prime}(y) .
\end{array}
$$

\section{Solución analítica.}

Se puede verificar que la solución analítica para este problema es:

$$
\begin{aligned}
& u_{1}(x, y)=8 f(x) g^{\prime}(y) \\
& u_{2}(x, y)=-8 f^{\prime}(x) g(y) \\
& p(\mathbf{x}, \operatorname{Re})=\frac{8}{\operatorname{Re}}\left[f^{\prime}(x) g^{\prime}(y)+F(x) g^{\prime \prime \prime}(y)\right]+64 F_{2}(x)\left[g(y) g^{\prime \prime}(y)-\left(g^{\prime}(y)\right)^{2}\right]
\end{aligned}
$$

en donde las funciones $F(x), F_{2}(x), f(x)$ y $g(y)$, así como sus derivadas, están definidas en (4.9). Observemos nuevamente que se cumple la condición $\int_{\Gamma} \mathbf{g} \cdot \mathbf{n} d \Omega=0$, ya que $\mathbf{n}$ es el vector normal en cada punto $(x, y)$ de la frontera de la cavidad y $\mathbf{g}(x, y)=\left(u_{1}(x, y), u_{2}(x, y)\right)$ representa la condición de frontera.

\section{Resultados numéricos}

Se resolvió numéricamente el problema (4.6)-(4.9) usando los métodos numéricos descritos en (3.15)-(3.17) para mallas uniformes con parámetro de discretización $h$ igual a $0.1,0.05,0.04$ y 0.025. Para cada una de estas mallas se resolvió el problema para números de Reynolds igual a $1,40,100,400,700$ y 1000 .

La Tabla 4-2 contiene un resumen de los resultados de las corridas. En dicha tabla se especifíca el número de iteraciones que se requieren para que el esquema de punto fijo converja con un error relativo menor o igual que $10^{-8}$ (veáse $(3.17)$ ), el incremento de tiempo $(\Delta t)$ que 
se utilizó en cada corrida, y el instante de tiempo final en que el esquema de Euler converge a la solución estacionaria con una precisión de $10^{-6}$ (veáse (3.15)). En ella se observa que la condición (3.14) para que el método iterativo de punto fijo converja se satisface. Más aún, $\Delta t$ puede ser más grande del indicado, como en los casos de $\operatorname{Re}=40$ en donde $\Delta t=0.2$. Sin embargo, esto puede provocar que el método de punto fijo realice más iteraciones de las que realizaría con un $\Delta t$ menor. Además, conforme el número de Reynolds crece se producen oscilaciones, las cuales pueden aumentar si $\Delta t$ es grande. Por lo tanto se recomienda que si se utiliza el esquema númerico propuesto en este trabajo para Re relativamente grandes se escoja un incremento del tiempo que cumpla la condición (3.14), para garantizar una mejor captura de las escalas del problema.

\begin{tabular}{|c|c|c|c|c|c|c|c|}
\hline \multicolumn{4}{|c|}{$\operatorname{Re}=1$} & \multicolumn{4}{|c|}{$\operatorname{Re}=40$} \\
\hline$h$ & Iter. Pto. Fijo & $\Delta t$ & $\mathbf{T}$ & $h$ & Iter. Pto. Fijo & $\Delta t$ & $\mathbf{T}$ \\
\hline 0.1 & 7 & .2 & 1.8 & 0.1 & 22 & .2 & 15.6 \\
\hline 0.05 & 7 & .2 & 1.8 & 0.05 & 22 & .2 & 15.6 \\
\hline 0.04 & 7 & .2 & 1.8 & 0.04 & 22 & .2 & 15.6 \\
\hline 0.025 & 7 & .2 & 1.8 & 0.025 & 22 & .2 & 15.6 \\
\hline \multicolumn{4}{|c|}{$R e=100$} & \multicolumn{4}{|c|}{$R e=400$} \\
\hline$h$ & Iter. Pto. Fijo & $\Delta t$ & $\mathbf{T}$ & $h$ & Iter. Pto. Fijo & $\Delta t$ & $\mathbf{T}$ \\
\hline 0.1 & 12 & .02 & 24.28 & 0.1 & 7 & .003 & 44.223 \\
\hline 0.05 & 11 & .02 & 24.30 & 0.05 & 7 & .003 & 15.228 \\
\hline 0.04 & 14 & .02 & 8.18 & 0.04 & 7 & .003 & 13.896 \\
\hline 0.025 & 11 & .02 & 0.4 & 0.025 & 7 & .003 & 4.98 \\
\hline \multicolumn{4}{|c|}{$\mathrm{Re}=700$} & \multicolumn{4}{|c|}{$R e=1000$} \\
\hline$h$ & Iter. Pto. Fijo & $\Delta t$ & $\mathbf{T}$ & $h$ & Iter. Pto. Fijo & $\Delta t$ & $\mathbf{T}$ \\
\hline 0.1 & \begin{tabular}{l|}
7 \\
\end{tabular} & .003 & 82.008 & 0.1 & 7 & .003 & 111.264 \\
\hline 0.05 & 7 & .003 & 26.697 & 0.05 & 7 & .003 & 24.597 \\
\hline 0.04 & 7 & .003 & 28.569 & 0.04 & 7 & .003 & 38.502 \\
\hline 0.025 & 7 & .002 & 11.827 & 0.025 & & & \\
\hline
\end{tabular}

Tabla 4-2: Resumen de los resultados de las corridas del problema de Navier-Stokes para la cavidad cuadrada.

Para verificar que el programa NAVSTOKE resuelve correctamente el problema, se realizó el análisis de convergencia para el problema descrito en (4.6)-(4.9). Las Figuras 4-4 y 4-5 muestran la superposición de las gráficas $\log (h)$ - $\log$ (error) para la velocidad y la presión, respectivamente. En ellas se observa que, para este problema, por lo menos se debe de utilizar 


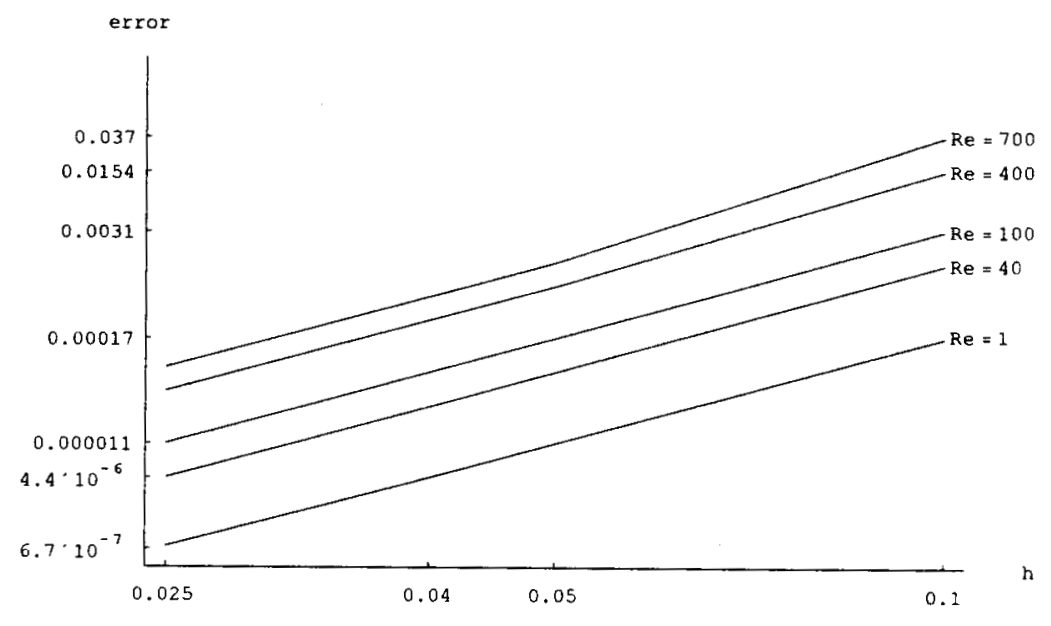

Figura 4-4: Grafica del error de la velocidad contra la resolución $h$ para diferentes números de Re.

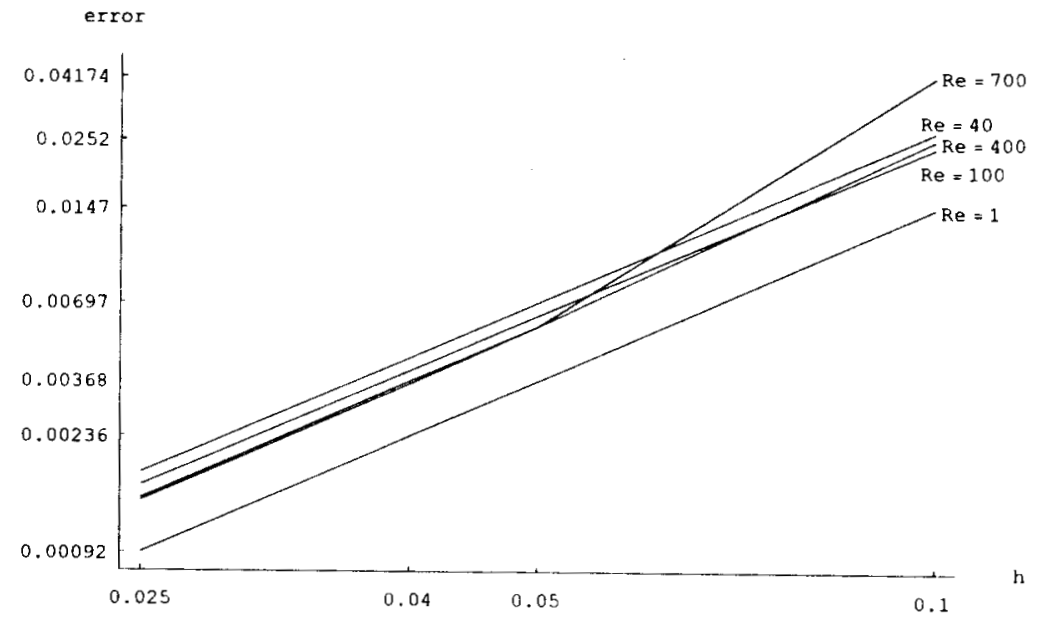

Figura 4-5: Grafica del error de la presión contra la resolución $h$ para diferentes números de Re. 
mallas con $h=0.05$ para asegurar que se obtengan buenos resultados tanto para la velocidad como para la presión. Cada gráfica se obtuvo al unir los cuatro puntos que se tienen cuando $h$ es igual a $0.1,0.04,0.05$ y 0.025 .

La Tabla 4-3 presenta un resumen de las pendientes para los diferentes números de Reynolds. Se obtuvieron de la siguiente forma: $\mathbf{m 1}$ se calculó de los primeros dos puntos $(h=0.1,0.05)$, m2 de los dos puntos intermedios $(h=0.05,0.04)$ y $\mathbf{m} 3$ de los puntos con $h=0.04,0.025 ; \mathbf{~ m p}$ es el promedios de las pendientes: $\mathbf{m p}=(\mathbf{m} 1+\mathbf{m} 2+\mathbf{m} 3) / 3$.

\begin{tabular}{|c|c|c|c|c|c|c|c|c|}
\hline \multicolumn{5}{|c|}{ Velocidad } & \multicolumn{4}{c|}{ Presión } \\
\hline $\mathbf{R e}$ & $\mathbf{m} \mathbf{1}$ & $\mathbf{m} \mathbf{2}$ & $\mathbf{m} \mathbf{3}$ & $\mathbf{m p}$ & $\mathbf{m} \mathbf{1}$ & $\mathbf{m} \mathbf{2}$ & $\mathbf{m} \mathbf{3}$ & $\mathbf{m p}$ \\
\hline 1 & 3,97330 & 3,99210 & 3,99806 & 3,99 & 1,99160 & 1,99640 & 1,99795 & 2,00 \\
\hline 40 & 4,13118 & 4,05107 & 4,02090 & 4,07 & 1,93889 & 1,97753 & 1,98753 & 1,97 \\
\hline 100 & 4,12992 & 4,05131 & 4,02229 & 4,07 & 1,92987 & 1,97615 & 1,98765 & 1,96 \\
\hline 400 & 4,46615 & 4,01106 & 4,02031 & 4,17 & 2,15230 & 1,98410 & 1,98996 & 2,04 \\
\hline 700 & 4,81285 & 3,99029 & 4,09726 & 4,30 & 2,87405 & 2,03469 & 2,00448 & 2,30 \\
\hline 1000 & 4,99291 & 3,84988 & & 2,95 & 3,58208 & 2,10403 & & 1,90 \\
\hline
\end{tabular}

Tabla 4-3: $\mathbf{m} 1 \mathbf{m} 2 \mathbf{m} 3$ son las pendientes de los 4 puntos de las gráficas de las Figs. 4-4 y $4-5$; mp es el promedio de las 3 pendientes.

Tomando como referencia la Tabla 4-3, se asegura que mp satisface las estimaciones de los errores para los elementos isoparamétricos tipo Hood-Taylor (2.19). Obsérvese que el error en el cálculo de la presión aumenta bruscamente para $\mathrm{Re}=400$ y 700 al ir de $h=0.05$ a $h=0.1$ (Fig. $4-5$ ) . Esto es debido a que la malla con $h=0.1$ es muy gruesa para resolver el problema con estos números de Reynolds, y no capta correctamente la escala del problema.

Con la intención de dar una idea gráfica de los resultados se crearon las Figuras 4-6 a la 4-8. La Fig. 4-6 exhibe los campos de velocidad de la solución aproximada y de la exacta del problema de Navier-Stokes, para una malla con $h=0.1$ y un número de Reynolds igual a 1000 . Cualitativamente se ve que los campos de velocidad de la solución aproximada y de la exacta representan el mismo comportamiento del flujo.

Las Figs. 4-7 y 4-8 contienen los contornos de presiones para una malla de 100 elementos $(h=0.1)$ y otra de 625 elementos $(h=0.04)$, así como los contornos de presión de la solución exacta. En la primera fila de gráficas, la Fig. 4-7 muestra los contornos de presión para $\mathrm{Re}=1$, en la segunda los de $R e=40$ y en la tercera para $R e=100$. Análogamente, cada fila de la Fig. 

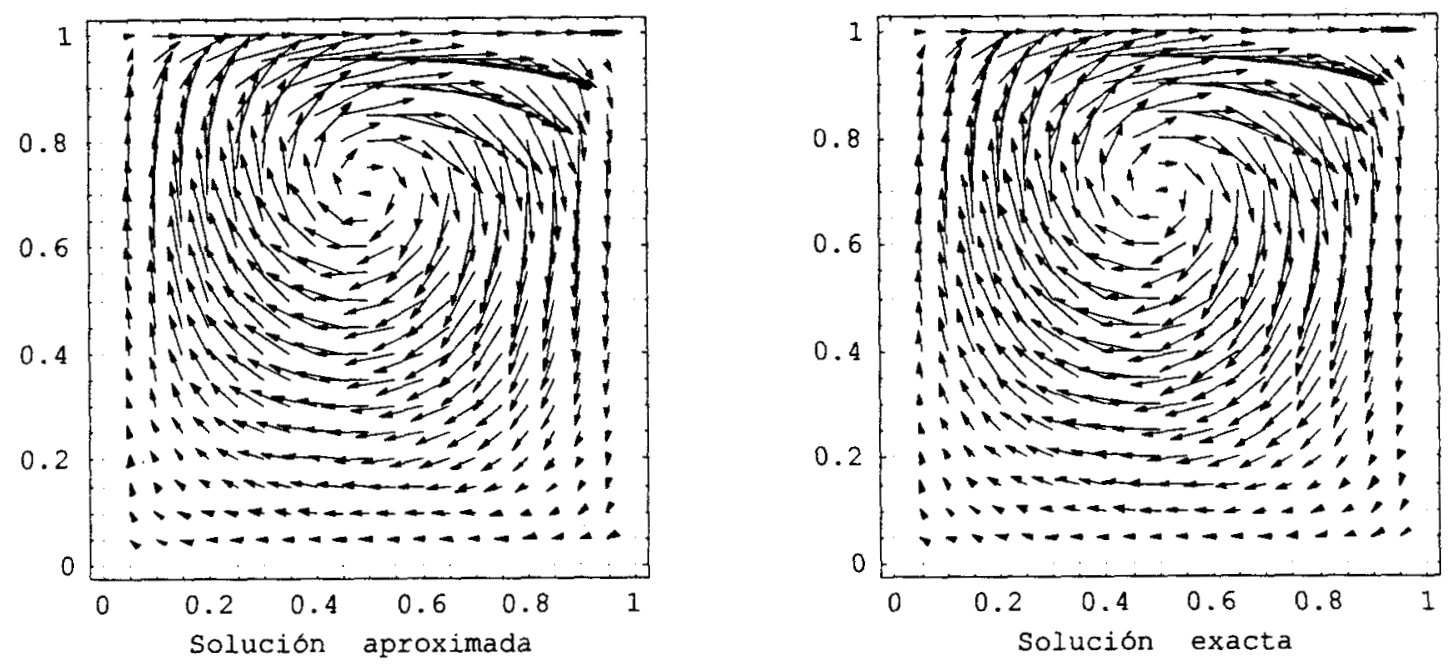

Figura 4-6: Campos de velocidades para el problema de Navier-Stokes. $\operatorname{Re}=1000, h=0.1$.

4-8 muestra los contornos de presión para $R e=400,700$ y 1000 , respectivamente.

Observando de la Fig. 4-7 los contornos de presión para $R e=1$ se ve que la solución aproximada no capta algunos contornos que aparecen en la parte superior de la cavidad y que si los hay en la exacta. En general podemos decir que los contornos de presión de la solución aproximada para una malla de 100 elementos $(h=0.1)$ están un poco "degenerados" con respecto a los de la solución exacta. En cambio, eso no ocurre para una malla de 625 elementos $(h=0.04)$, la gráficas de las soluciones aproximadas y de las exactas que están en las Figs. 4-7 y 4-8 son prácticamente las mismas. Más aún, una malla de 625 elementos puede captar pequeños contornos que una malla de 100 elementos no lo hace, ver por ejemplo las gráficas asociadas a $\mathrm{Re}=400$. Esto es consistente con lo observado en la Figura 4-5.

Los resultados anteriores nos muestran que el método utilizado produce buenos resultados tanto para encontrar velocidades y presiones si se utilizan los parámetros de discretización adecuados. 
a. $h=0.1$
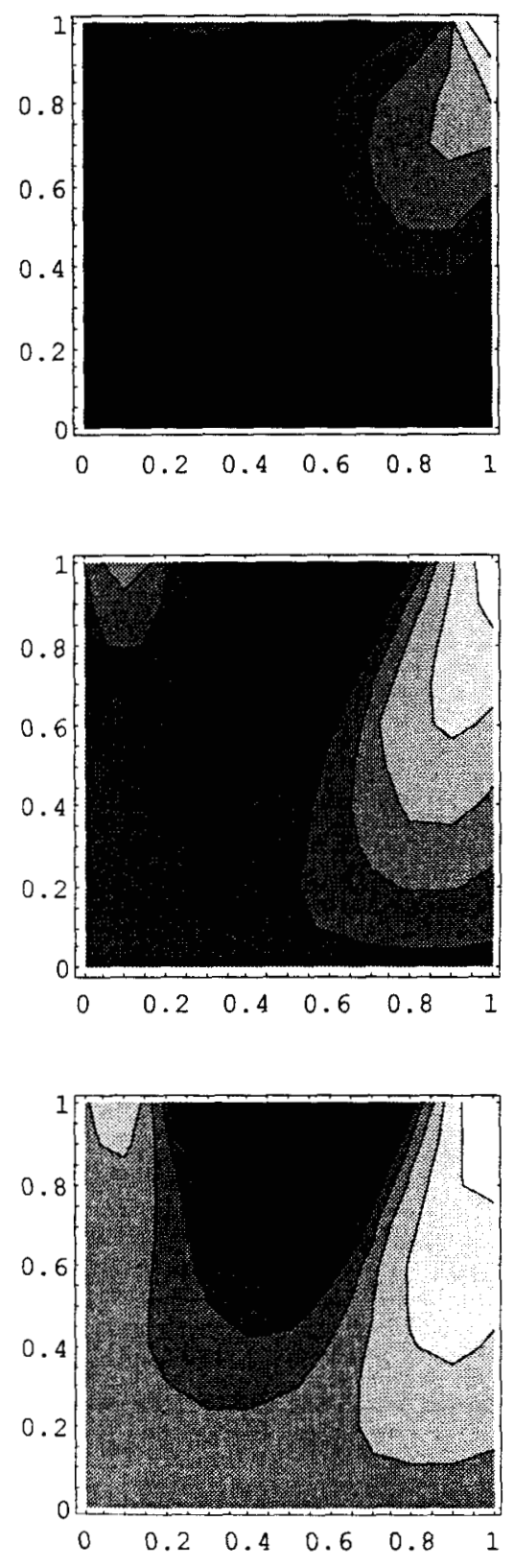

b. $h=0.04$
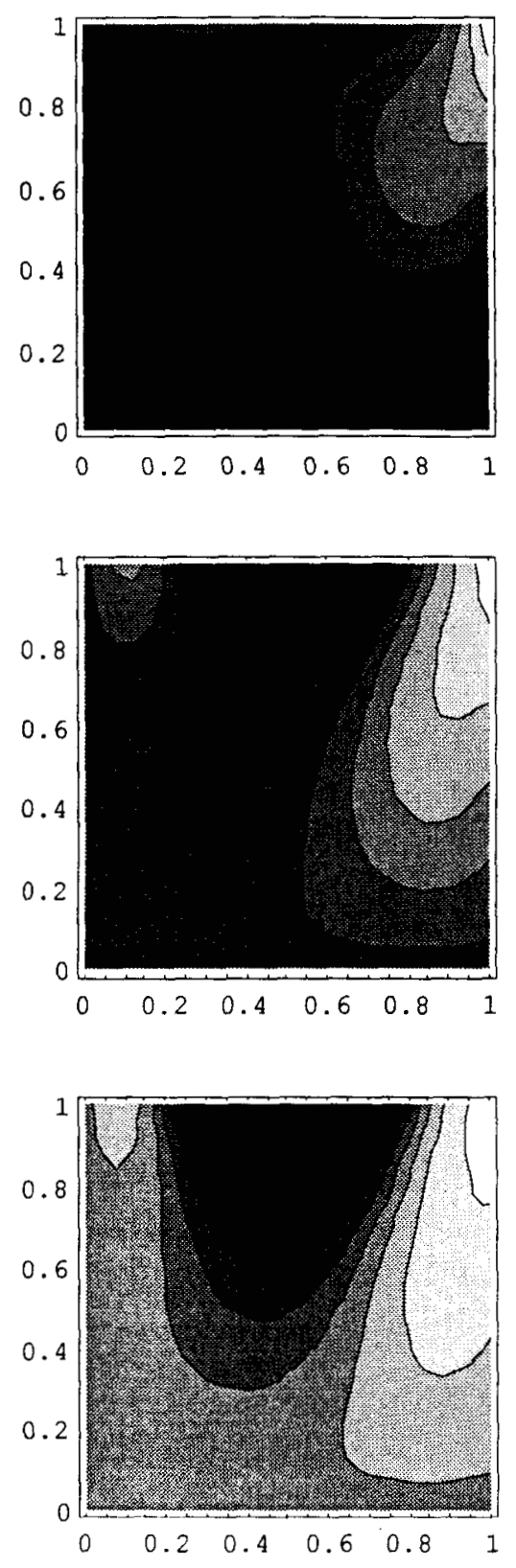

c. Sol exacta
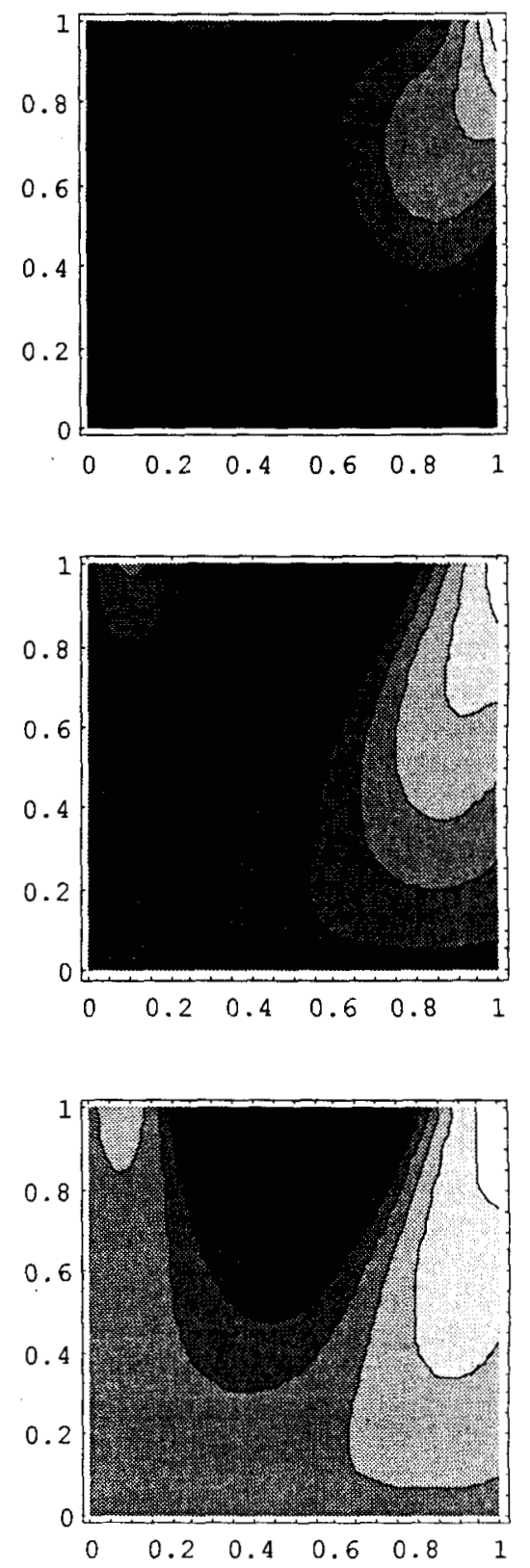

Figura 4-7: Contornos de presión para N-S obtenidos usando mallas de : a. $h=0.1$; b. $h=0.04$; c. Solución exacta. Para $R e=1,40,100$ (veáse de arriba hacia abajo). 
a. $h=0.1$
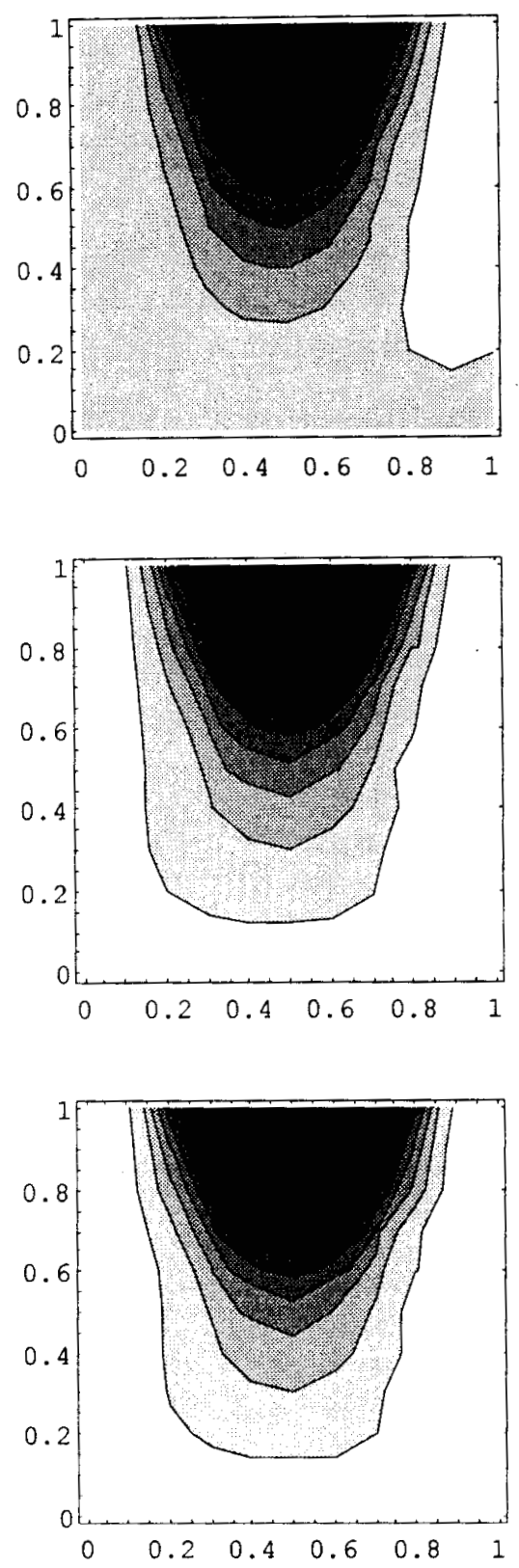

b. $h=0.04$
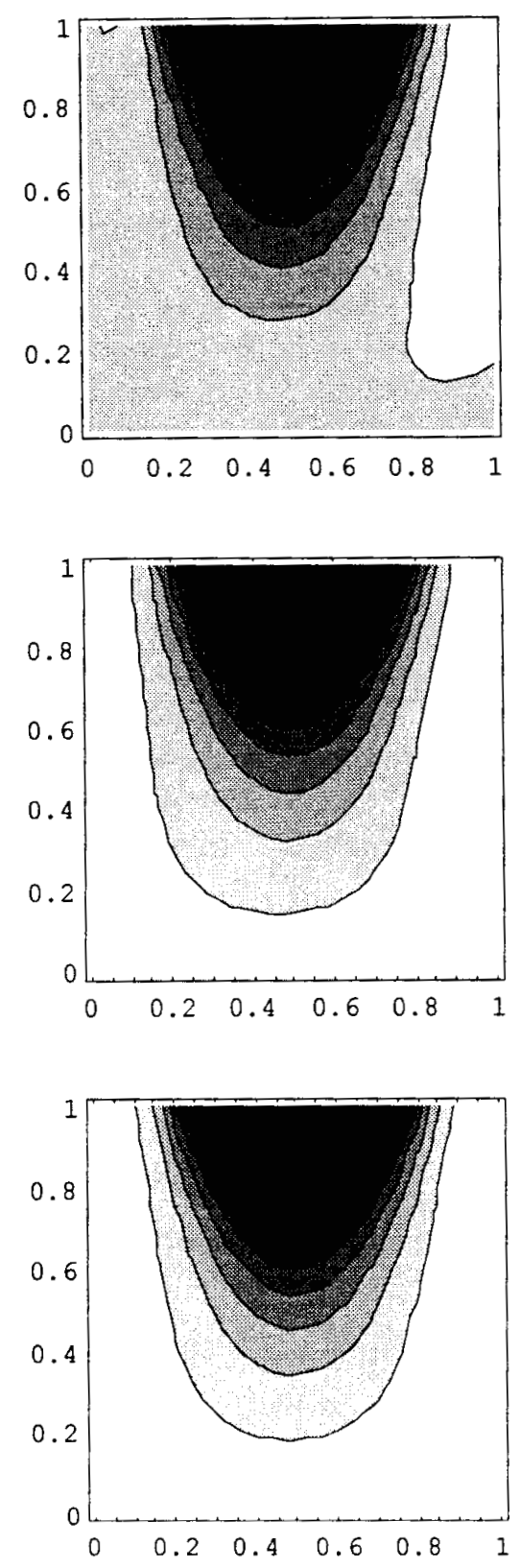

c. Sol - exacta
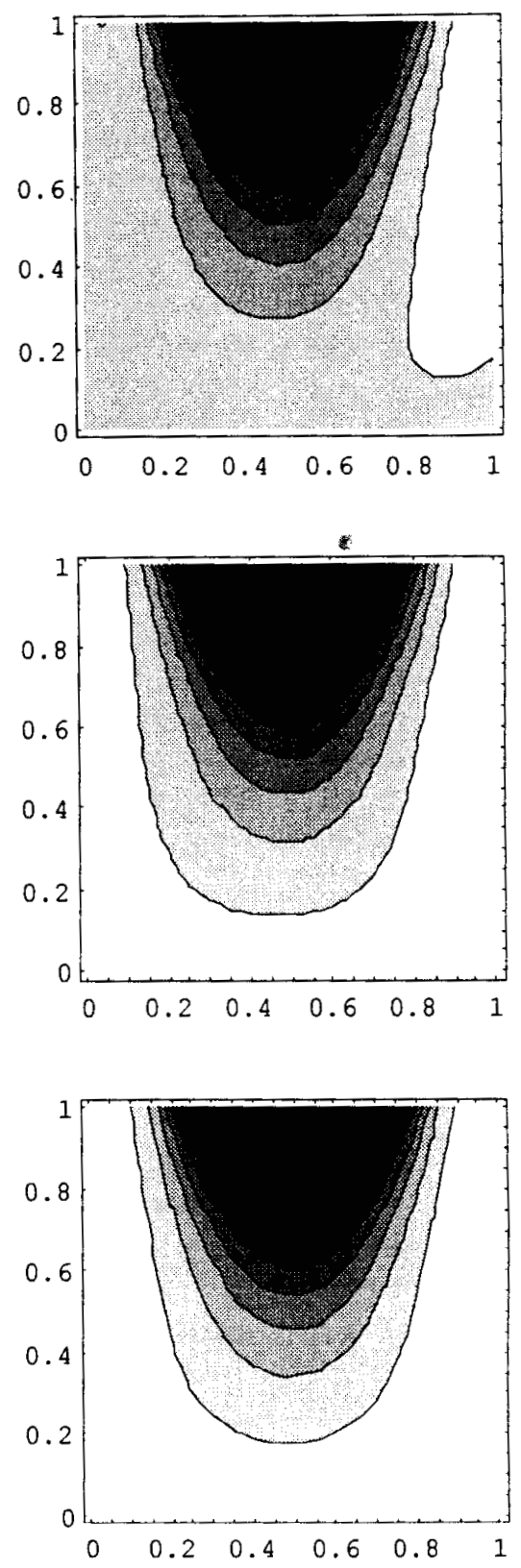

Figura 4-8: Contornos de presión para N-S obtenidos usando mallas de : a. $h=0.1$; b. $h=0.04$; c. Solución exacta. Para $R e=400,700,1000$ (veáse de arriba hacia abajo). 


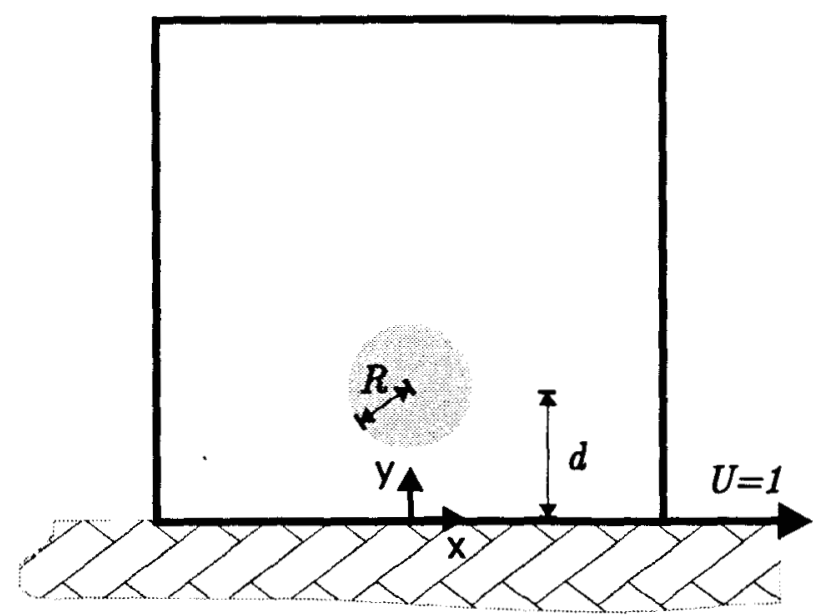

Figura 4-9: Dominio computacional : altura $=2$, ancho $=2, R=0.25$ y $d=0.5$.

\subsection{Aplicación al flujo alrededor de un cilindro cerca de una pared en movimiento.}

Consideremos el flujo de Stokes en dos dimensiones de un fluido Newtoniano, isotérmico, estacionario, incompresible y muy viscoso, que fluye alrededor de un cilindro horizontal situado cerca de una pared en movimiento (veáse Fig. 4-9). Este es un problema de prueba más complejo que el anterior (veánse Maslanik [19] y Wannier [28]), y la solución varía dependiendo de la distancia de separación entre el cilindro y la pared. En este trabajo se fijó al radio del cilindro en 0.25 y la separación que hay entre él y la pared es de 0.5 unidades. Este es un ejemplo de un problema de lubricación y representa el caso límite de un lubricante entre dos cilindros excéntricos en donde el cilindro interior está fijo y el exterior se mueve en sentido contrario a las manecillas del reloj, con radio infinito.

Para resolver este problema numéricamente, se delimita la región computacional a una cavidad cuadrada de dos unidades de longitud por lado, como se ejemplifica en la Figura 4-9.

Las ecuaciones que modelan este problema son las ecuaciones de Stokes: 


$$
\begin{gathered}
\mu \Delta \mathbf{u}=\nabla p, \\
\nabla \cdot \mathbf{u}=0, \\
\mathbf{u}=0 \text { sobre } \quad(y-d)^{2}+x^{2}=R^{2}, \\
\mathbf{u}=(U, 0) \text { sobre } y=0 \quad \text { cuando } y^{2}+x^{2} \rightarrow \infty
\end{gathered}
$$

donde $\mathbf{u}=(u, v)$ es el campo de velocidades, $p$ es la presión, $\mu=1$ es la viscosidad del fluido, $U=1$ es la magnitud constante de la velocidad en la pared y en el infinito, $d(=0.5)$ es la distancia del centro del cilindro a la pared y $R(=0.25)$ es el radio del cilindro. Observemos que cuando nos restringimos al dominio computacional de la Fig. 4-9, se respetan las condiciones de frontera del cilindro y la pared, pero los lados restantes del cuadrado tendrán condiciones de frontera tipo Dirichlet iguales al valor de la solución analítica (veáse la ec. 4.12).

\subsubsection{Solución analítica.}

La solución analítica que se presenta a continuación es un caso límite de la solución obtenida por Wannier [28] para el flujo de Stokes que pasa entre dos cilindros excéntricos rotando, la cual se puede consultar en Maslanik [19]. Los campos de velocidades $(u, v)$ y de presiones $p$ están dados por:

$$
\begin{aligned}
& u=-\frac{2}{K_{1}}(A+F y)\left[s+y+\frac{K_{1}}{K_{2}}(s-y)\right]-F \ln \left(\frac{K_{1}}{K_{2}}\right) \\
& -\frac{B}{K_{1}}\left[s+2 y-\frac{2 y(s+y)^{2}}{K_{1}}\right]-\frac{C}{K_{2}}\left[s-2 y+\frac{2 y(s-y)^{2}}{K_{2}}\right]+U, \\
& v=\frac{2 x}{K_{1} K_{2}}(A+F y)\left(K_{2}-K_{1}\right)-\frac{2 B x y}{K_{1}^{2}}(s+y)-\frac{2 C x y}{K_{2}^{2}}(s-y) \text {, } \\
& p=-\frac{4 B x(s+y)}{K_{1}^{2}}-\frac{4 C x(s-y)}{K_{2}^{2}}-\frac{16 F s x y}{K_{1} K_{2}}
\end{aligned}
$$

en donde

$$
\begin{gathered}
K_{1}=x^{2}+(s+y)^{2}, \quad K_{1}=x^{2}+(s-y)^{2}, \quad \Gamma=\left(\frac{d+s}{d-s}\right), \quad s=\sqrt{\left(d^{2}-R^{2}\right)}, \\
A=\frac{-d U}{\ln (\Gamma)}, \quad B=\frac{2(d+s) U}{\ln (\Gamma)}, \quad C=\frac{2(d-s) U}{\ln (\Gamma)}, \quad F=\frac{U}{\ln (\Gamma)} .
\end{gathered}
$$




\subsubsection{Solución numérica.}

Se resolvió numéricamente este problema para una malla de 400 elementos $(h=0.05)$, la cual se presenta en la Figura 4-10. Debe mencionarse que para construir la malla se fijó el origen en el centro del cilindro. Los resultados que se presentan se obtuvieron fijando el nodo con coordenadas $(0,0.25)$ y con un valor para la presión de -5.260762888816203 , que se obtuvo de la solución analítica. Se impusieron condiciones de frontera tipo Dirichlet en la frontera de la cavidad usando la solución exacta, excepto para la frontera que coincide con la pared (lado inferior del cuadrado) en donde $\mathbf{u}=(1,0)$. El error relativo entre la solución aproximada y la exacta para la velocidad fue de 0.00068043 y para la presión fue de 0.02108223 . Debemos de observar que se hicieron varias corridas fijando diferentes nodos para la presión y los resultados fueron los mismos.

La Figura 4-11 muestra que cualitativamente las gráficas de los campos de velocidades para las soluciones aproximada y exacta son virtualmente los mismas. La Figura 4-12 exhibe los contornos de presión de las soluciones del problema tanto para la aproximada como para la exacta. Al observar la Figura se ve que la gráfica de la solución aproximada tiene en la parte inferior unos pequeños contornos que en la gráfica de la exacta no aparecen. Esto se debe a que la malla en esa región es un poco gruesa y tendría que refinarse ahí para obtener mejores aproximaciones de la presión.

Los resultados numéricos reportados en las Figuras 4-11 y 4-12 determinan que la implantación de la familia de elementos isoparamétricos mixto en el programa NAVSTOKE funciona corretamente.

\subsection{Implantación.}

El programa NAVSTOKE se escribió en lenguaje Fortran y los problemas presentados se corrieron en una Power Challenge con 18 procesadores R8000 y sistema operativo IRIX 6.2; y en una Origin 200 de Silicon Graphics con 4 procesadores R10000 y sistema operativo IRIX 6.4. 


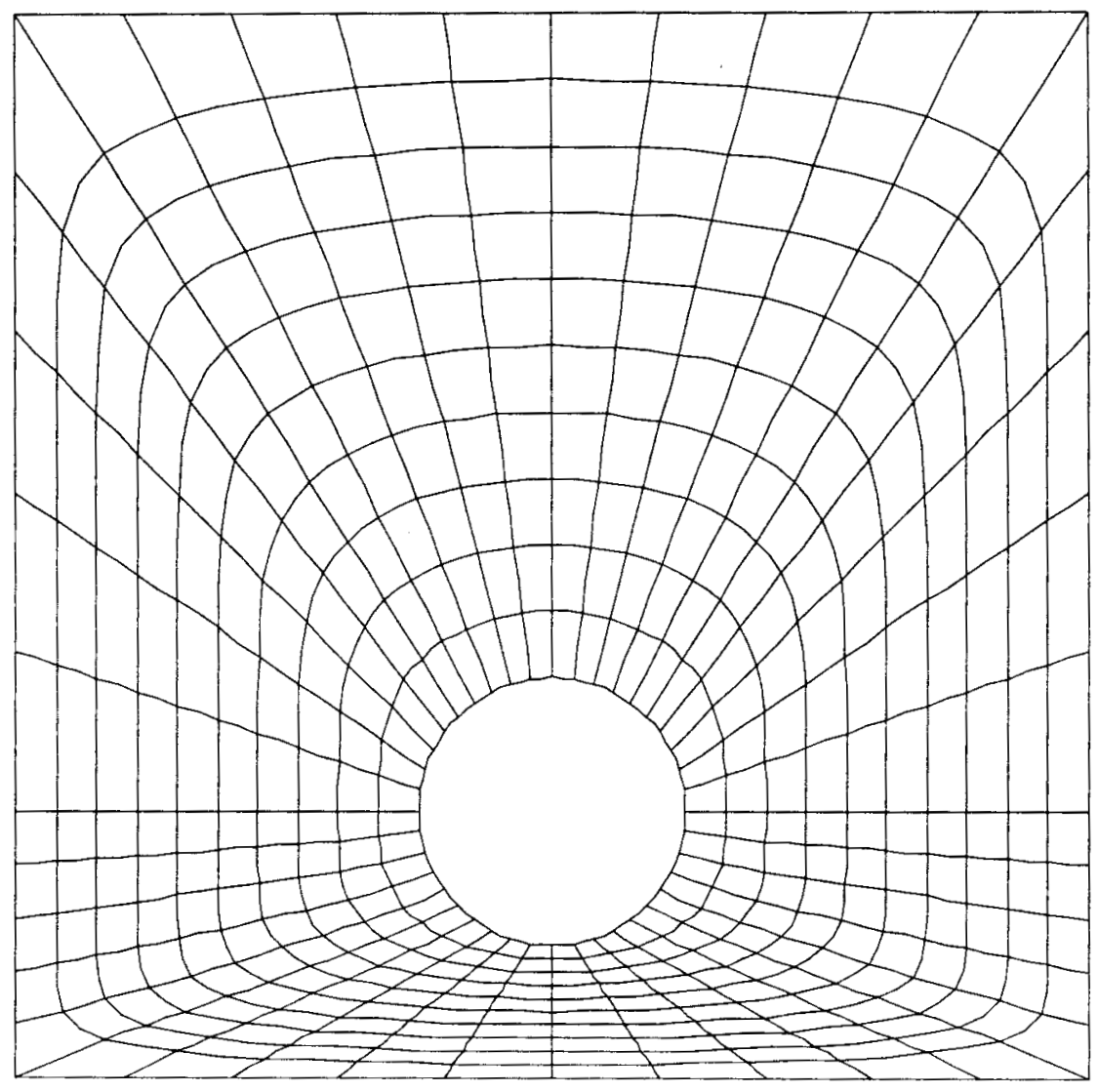

Figura 4-10: Mallado de la región computacional del problema de un fluido viscoso que fluye alrededor de un cilindro cerca de una pared en movimiento. 

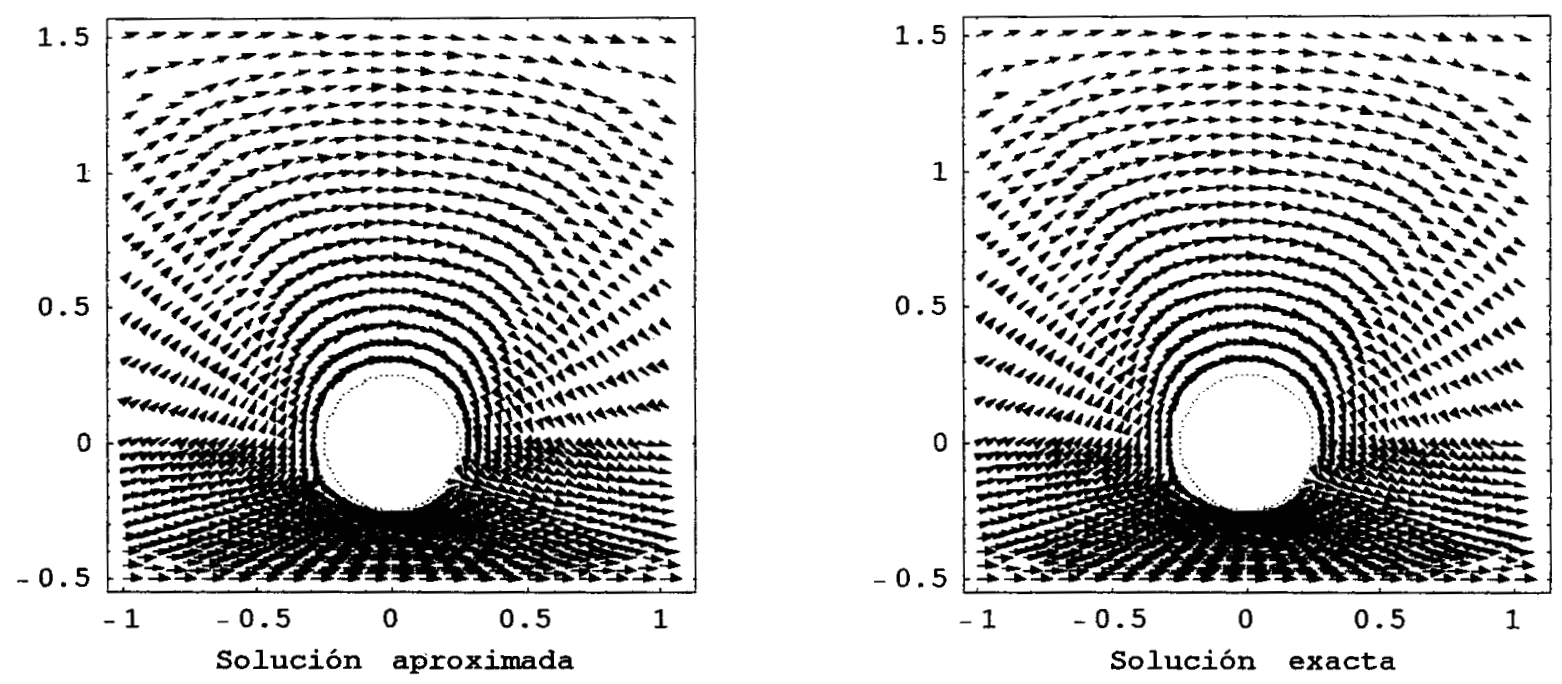

Figura 4-11. Campos de velocidades para el flujo que pasa alrededor de un cilindro cerca de una pared en movimiento. $\mathrm{Re}=1$, malla de 400 elementos.
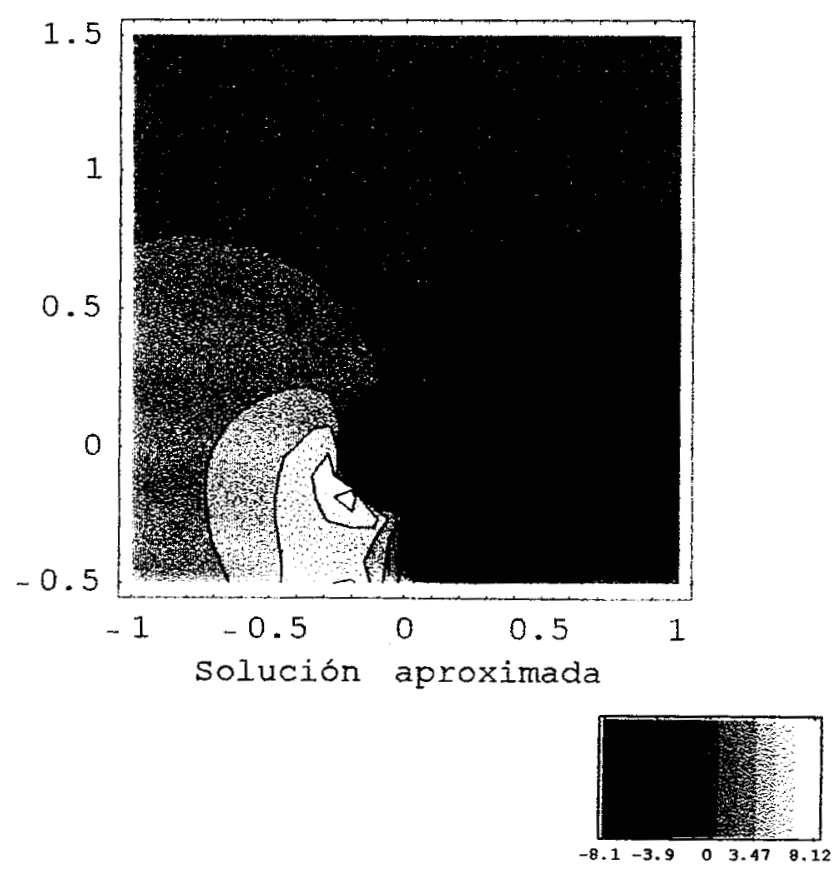
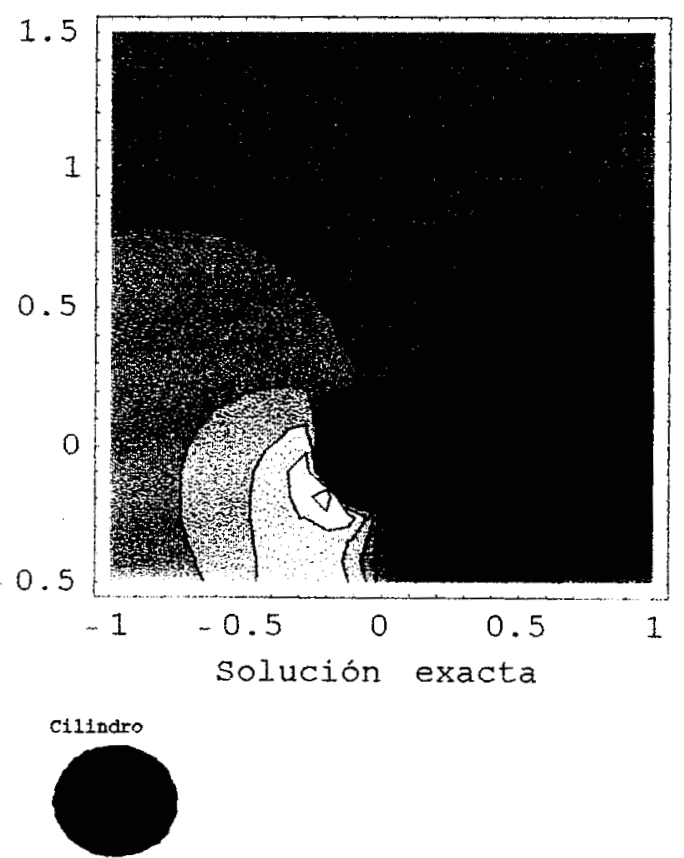

Figura 4-12. Contornos de presión para el flujo que pasa alrededor de un cilindro cerca de una pared en movimiento. 


\section{Conclusiones}

Se elaboró un programa que resuelve numéricamente las ecuaciones de Stokes y Navier-Stokes para fluidos homogéneos, isotérmicos, incompresibles y viscosos sobre dominios en el plano con fronteras curvas y con condiciones de frontera tipo Dirichlet o Neumann. Para ello, se aplicó el método de elemento finito en combinación con un esquema implícito de Euler hacia atrás que es estable [26] y un esquema iterativo de punto fijo que es convergente [15]. Además, para aproximar simultáneamente a la velocidad y a la presión se utilizó un elemento de referencia mixto tipo Taylor-Hood y una familia de elementos isoparamétricos mixtos.

Se mostró la necesidad de usar un esquema no estacionario para resolver los problemas estacionarios para diferentes fluidos viscosos. Ya que si se incorpora el término temporal a la modelación aumentar el radio de convergencia del método de punto fijo.

Se verificó que el software desarrollado funciona correctamente por medio de dos aplicaciones. Para la primera aplicación, el flujo sobre una cavidad cuadrada, se reportaron las gráficas de convergencia de $\log (h)$ - $\log ($ error $)$ para la velocidad y la presión. Se corroboró que satisfacen las estimaciones de los errores de los elementos isoparamétricos tipo Hood-Taylor dadas por Stenberg [25]. La segunda aplicación que es más compleja que la anterior, es un flujo alrededor de un cilindro cerca de una pared en movimiento. Como su dominio es no trivial se usó este problema para comprobar que la implantación de los elementos isoparamétricos es correcta. Los resultados numéricos de ambas aplicaciones se compararon con las soluciones analíticas y se presentaron las gráficas de los campos de velocidades y de los contornos de presión. Además se verificó en la primera aplicación que el problema de Stokes se puede usar 
para modelar el comportamiento de fluidos muy viscosos.

En [15] se muestra que el esquema iterativo de punto fijo converge si el incremento de tiempo está acotado por el inverso del número de Reynolds (veáse (3.14)). Se hicieron pruebas numéricas y se ha mostrado que el método iterativo convege para incrementos de tiempos más grandes que la cota teórica. Sin embargo las soluciones se deterioran debido a que cuando $\Delta t$ es relativamente grande se producen soluciones transitorias oscilatorias persistentes, sobre todo cuando Re es relativamente grande. Esto provoca que el número de iteraciones en el tiempo para que la solución converja al estado estacionario aumente. Se observa que, en general, este fenómeno se puede controlar si se escoge $\Delta t$ de manera adecuada. El hecho de que la relación (3.14) indique el valor adecuado de $\Delta t$ no es mero accidente. Se sabe que para números de Re muy grandes el término convectivo $\mathbf{u} \cdot \nabla \mathbf{u}$ en la ecuación de Navier-Stokes domina el problema $\mathrm{y}$, por tanto, los parámetros de discretización $\Delta t \mathrm{y} h$ deben ser lo suficientemente pequeños para captar las escalas del mismo. En particular la rapidez de convección solo puede simularse de manera adecuada si $\Delta t$ es suficientemente pequeño.

También se verificó que el método mixto de tipo Taylor-Hood da buenas aproximaciones tanto para la velocidad como para la presión. Un simulador numérico aceptable debe producir resultados en los que se obtienen al menos ordenes de convergencia de grado dos. La Tabla 4-3 muestra que este es el caso para el método utilizado en este trabajo.

Como posible trabajo futuro, de manera más inmediata, se requiere incorporar un método especial de solución de sistemas lineales con matrices ralas, con el fin de aprovechar la estructura del sistema y ahorrar una considerable cantidad de memoria. En este trabajo no se utilizó ninguna técnica especial para sistemas con matrices ralas para resolver los sistemas lineales resultantes. Esta es la principal desventaja del programa NAVSTOKE.

Se pretende que este software sirva como base para resolver problemas más complejos que involucren fluidos viscosos estacionarios y no estacionarios, como los que se presentan en los problemas de frontera libre acoplados con la ecuación de energía. 


\section{Bibliografía}

[1] Batchelor G.K. An introduction to Fluid Dynamics. Cambridge University Press, 1991.

[2] Carey Graham F., Oden J. Tinsley. Finite Elements. Fluid Mechanics. Volume VI. Prentice Hall, 1986.

[3] Chorin A.J., Marsden J.E. A Mathematical Introduction to Fluid Mechanics. SpringerVerlag, New York Inc, 1990.

[4] Ciarlet Philippe G. The Finite Element Method for Elliptic Problems. Studies in Mathematics and its Aplications. North-Holland Publishing Company Amsterdam.New York·Oxford, 1978.

[5] Currie I. G. Fundamental Mechanics of Fluids. Mc Graw-Hill, 1974.

[6] Cuvelier C., Segal A. and A.A. van Steenhoven. Finite Element Methods and Navier-Stokes Equations. D. Reidel Publishing Company, Mathematics and its Applications, 1988.

[7] FIDAP. Fluid Dynamics International, INC. Revision 6.0. 500 Davis st, Suit 600 Evanston, Illinois 60201 (U.S.A), 1991.

[8] Girault Vivette, Raviart Pierre-Arnoud. Finite Element Methods for Navier-Stokes Equations. Springer Series in Computational Mathematics 5, 1986.

[9] Gunzberger Max D. Finite Element Methods for Viscous Incompressible Flows. Computer Science and Scientific Computing, 1989.

[10] Heywood J.G., Masuda K, Rautmann R., V.A. Slonnikov. The Navier-Stokes Equations. Theory and Numerical Methods. Lecture Notes in Mathematics. Springer-Verlag, 1988. 
[11] Heywood J.G., K Masuda, R. Rautmann, Slonnikov V.A.. The Navier-Stokes Equations II. Theory and Numerical Methods. Lecture Notes in Mathematics. Springer-Verlag, 1991.

[12] Hughes Willian F. An Introduction to Viscous Flow. Hemisphere Publishing Corporation, 1979.

[13] Hunter Rouse. Elementary Mechanics of Fluids. Dover, 1978.

[14] Johnson Claes. Numerical Solution of Partial Differential Equations by the Finite Element Method. Cambridge University Press, 1992.

[15] Juárez-Valencia L. Héctor. Numerical Simulation of Time-Dependent Viscous Flows in Complex Geometries. Research Report UH/md -224. Department of Mathematics University of Houston (1996).

[16] Karakashian Ohannes A. On a Galerkin-Lagrange Multiplier Method for the Stationary Navier-Stokes Equations. SIAM J. Numer. Anal. Vol 19,No. 5, 909-923 (1982).

[17] Klouček Petr, Franz S. Rys. Stability the Fractional Step $\theta$-scheme for the Nonstationary Navier-Stokes Equations. SIAM J. Numer. Anal.. Vol31, No. 5, 1312-1335 (1994).

[18] Maslanik Miriam K., Sani R. L. y Gresho P. M. An Isoparametric Finite-Element Stokes Flow Test Problem. Communications in Applied Numerical Methods. Vol. 6, 429-436 (1990).

[19] Oden J. Tinsley, Eric B. Becker y Graham f. Carey. Finite Elements an Introduction. Volume I. Prentice-Hall, 1981.

[20] Oden J. Tinsley, Eric B. Becker y Graham f. Carey. Finite Elements. Mathematical Aspects. Volume IV. Prentice-Hall, 1983.

[21] Perronnet A. Les Méthodes de Resolution des Systemes Lineaires. Leur Technique de Stockage. Universite Pierre et Marie Curie. Laboratoire Analyse Numérique. No. Enregistrement 81022 .

[22] Saavedra Barrera P. Paquete Malla. Reporte Técnico. Universidad Autónoma Metropolitana, 1989. 
[23] Saavedra P. and L.R. Scott. Variational Formulation of a Model Free-Boundary Problem, Math. Comp.. Vol 57. Num 196. 451-475 (1991).

[24] Shih T. M., Tan C. H., B. C. Hwang. Effects of Grid Staggering on Numerical Schemes. International Journal for Numerical Methods in Fluids. Vol 9. 193-212 (1989).

[25] Stenberg Rolf. Analysis of Mixed Finite Element Methods for the Stokes Problem: A Unified Aproach. Mathematical of Computation. Vol. 42, No. 165, 9-23 (1984).

[26] Temam Roger. Navier-Stokes Equations. Theory and Numerical Analysis. North-Holland, 1984.

[27] Wannier Gregory H. A Contribution to the Hydrodynamics of Lubrication. Quarterly of Applied Mathematics. Vol. VIII, No. 1, April (1950). 